Portland State University

PDXScholar

Summer 8-2-2017

\title{
The Cul3 Ubiquitin Ligase: an Essential Regulator of Diverse Cellular Processes
}

Brittney Marie Davidge

Portland State University

Follow this and additional works at: https://pdxscholar.library.pdx.edu/open_access_etds

Part of the Biology Commons, and the Cell Biology Commons

Let us know how access to this document benefits you.

\section{Recommended Citation}

Davidge, Brittney Marie, "The Cul3 Ubiquitin Ligase: an Essential Regulator of Diverse Cellular Processes" (2017). Dissertations and Theses. Paper 3782.

https://doi.org/10.15760/etd.5666

This Dissertation is brought to you for free and open access. It has been accepted for inclusion in Dissertations and Theses by an authorized administrator of PDXScholar. Please contact us if we can make this document more accessible: pdxscholar@pdx.edu. 
The Cul3 Ubiquitin Ligase: An Essential Regulator of Diverse Cellular Processes

by

Brittney Marie Davidge

A dissertation submitted in partial fulfillment of the requirements for the degree of

\author{
Doctor of Philosophy \\ in \\ Biology
}

\author{
Dissertation Committee: \\ Jeffrey Singer, Chair \\ Justin Courcelle \\ Kenneth Stedman \\ James McCormick \\ Michael Bartlett \\ Dirk Iwata-Reuyl
}

Portland State University

2017 
(C) 2017 Brittney Marie Davidge 


\begin{abstract}
:
Cul3 forms E3 ubiquitin ligase complexes that regulate a variety of cellular processes. This dissertation describes Cul3's role in several of these pathways and provides new mechanistic details regarding the role of Cul3 in eukaryotic cells. Cyclin $\mathrm{E}$ is an example of a protein that is regulated in a Cul3-dependent manner. Cyclin E is a cell cycle regulator that controls the beginning of DNA replication in mammalian cells. Increased levels of cyclin E are found in some cancers, in addition, proteolytic removal of the cyclin E N-terminus occurs in some cancers and is associated with tumorigenesis. Cyclin E levels are tightly regulated and controlled in part through ubiquitin-mediated degradation initiated by one of two E3 ligase complexes, Cul1 and Cul3. Cul1 mediated degradation of cyclin E is triggered by cyclin E phosphorylation, however the mechanism Cul3 uses to ubiquitinate cyclin E is poorly understood. In order to gain a better understanding of how Cul3 mediates cyclin E destruction we identified the degron on cyclin E that is important in Cul3 dependent degradation. In addition, we show this degron is lacking in LMW cyclin E (found in abundance in breast cancer), providing a novel mechanism for how these cyclin E modifications result in increased cyclin E levels by avoiding the Cul3 degradation pathway.
\end{abstract}




\section{ACKNOWLEDGMENTS:}

I would like to thank my advisor, Dr. Jeff Singer, for helping me to learn the necessary skills to succeed in science. I would also like to thank the other members of my dissertation committee for their advice and guidance as well as the other members of the Singer lab for their friendship and support. I would like to acknowledge the staff of the Biology department at Portland State for their support while I worked as a teaching assistant. I am forever grateful for my husband, Sean Sweeney, as he has demonstrated unconditional support and patience while I worked to finish my degree. I am grateful for all of my friends, family, and fellow students (both graduate and undergraduate) who have supported me throughout my graduate school journey. Lastly, I need to thank my pet Cockatiel, Cosmo, for always being such a bright ray of sunshine. 


\section{TABLE OF CONTENTS}

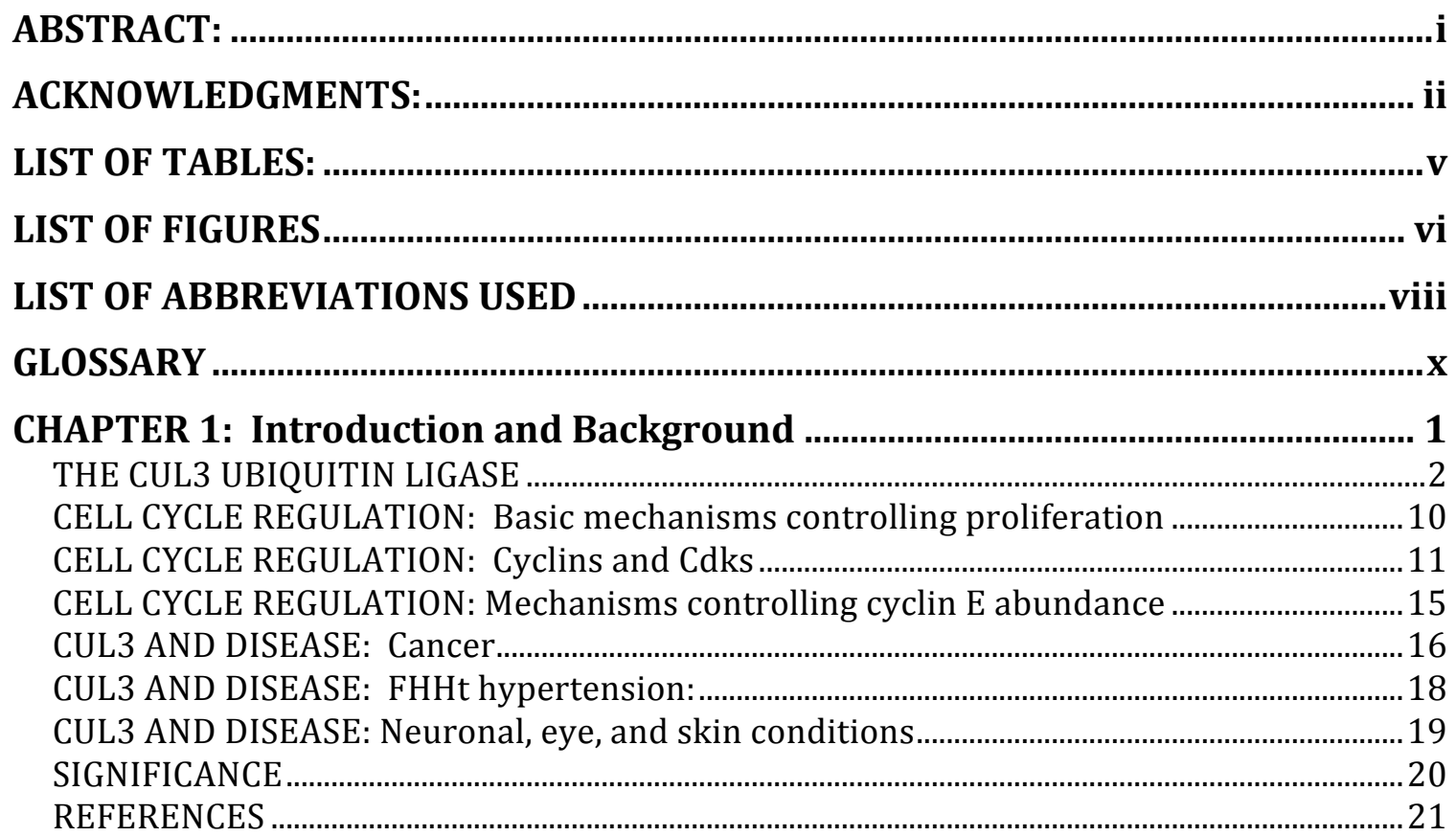

\section{CHAPTER 2: The N-terminus of Cyclin E is Required for Cul3-mediated}

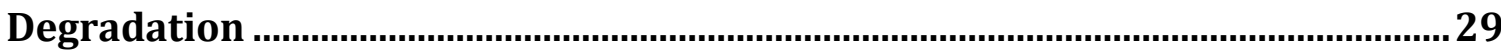

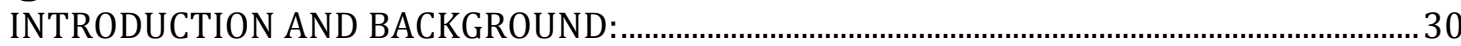

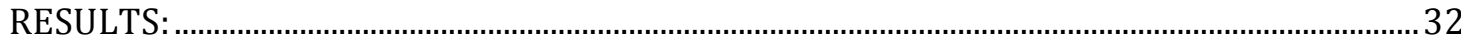

Cyclin E binds directly to Cul3 independently of BTB domain-containing proteins........32

Mutations in the N-terminal region of cyclin E prevent degradation by the Cul3

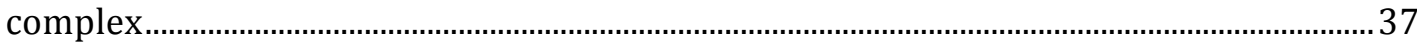

Lysine 48 on cyclin E serves as a ubiquitination site for Cul3 ……........................................ 42

The $\mathrm{N}$-terminal domain of cyclin $\mathrm{E}$ is required for degradation ............................................45

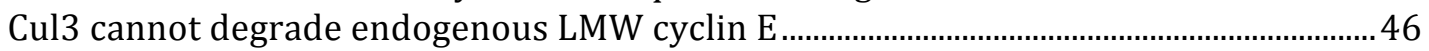

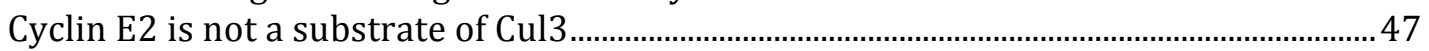

Loss of Cul3 results in early accumulation of cyclin E after release from serum

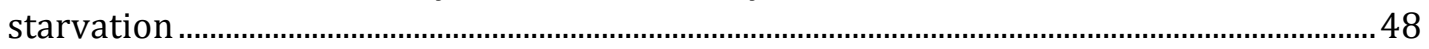

RhoBTB3 interacts with UbE2E1 to facilitate complex formation ......................................52

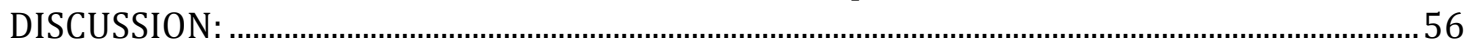

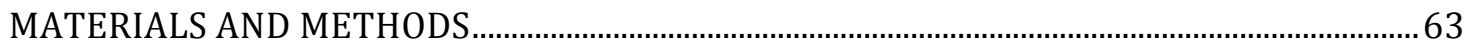

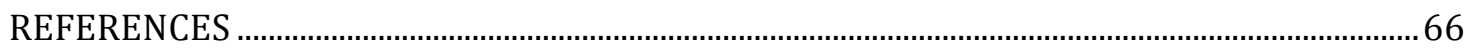

CHAPTER 3: Identifying Possible Roles of Cul3 and Klhl3 in the Kidney ......... 70

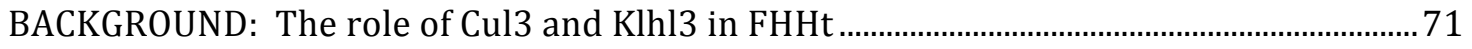

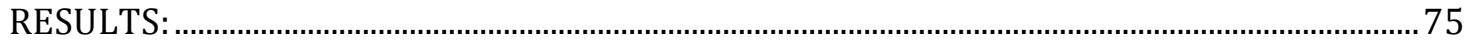

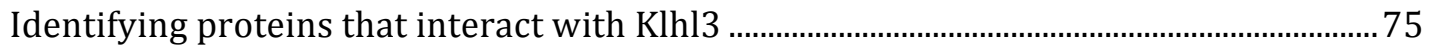

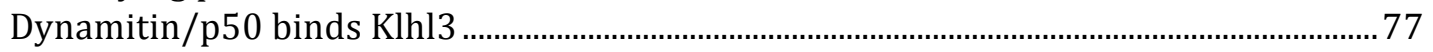

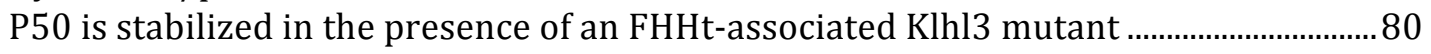

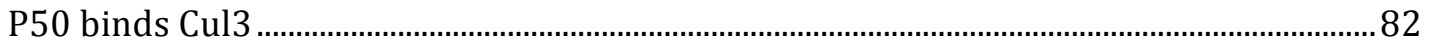

Cul3 and Klhl3 ubiquitinate p50/dynamitin in vitro ............................................................. 83 


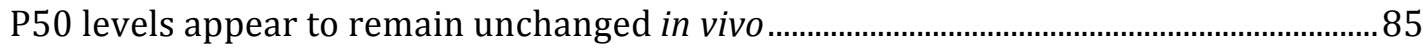

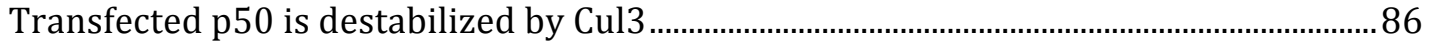

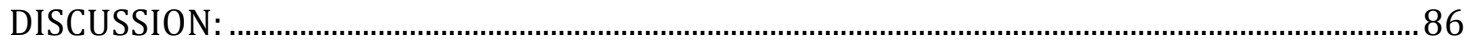

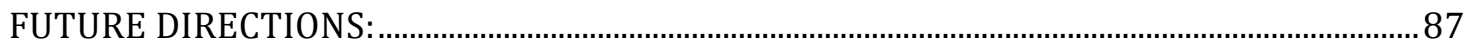

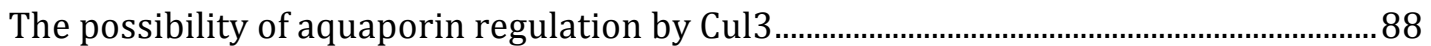

Other identified proteins that may interact with Klhl3 ……...................................................94

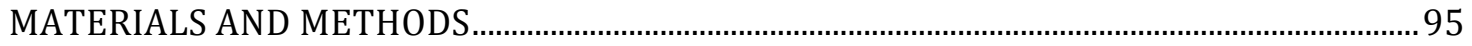

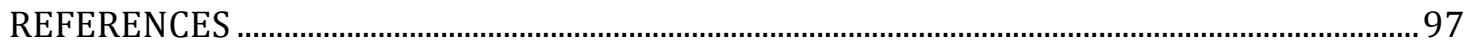

CHAPTER 4: Conclusions, Discussion, and Future Directions........................... 101

RESEARCH QUESTION 1: What is the nature of the interactions between Cul3, Cdk2, and

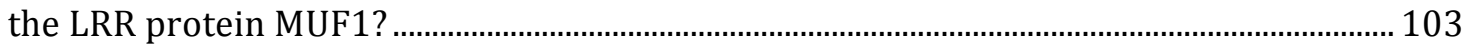

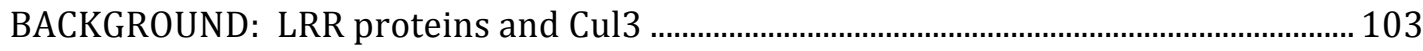

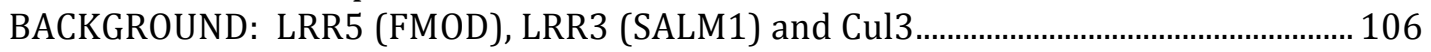

BACKGROUND: LRR5 (FMOD) interacts with the BTB protein FAZF ............................... 107

RESULTS: Exploring the interactions between the LRR protein MUF1, Cul3, and Cdk2

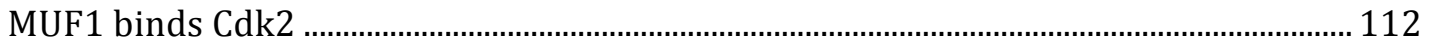

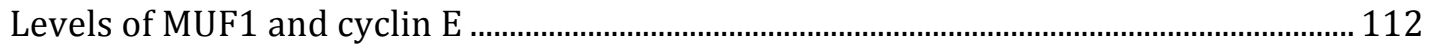

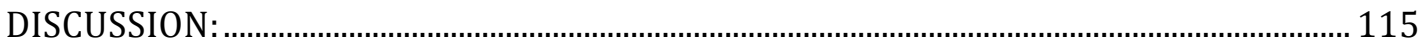

RESEARCH QUESTION 2: How does loss of Cul3 affect breast cancer cells? ........................ 118

RESEAERCH QUESTION 3: What other modifications may affect cyclin E in vivo?........... 119

RESEARCH QUESTION 4: How do BTB proteins affect the binding of E2 enzymes to Cul3-

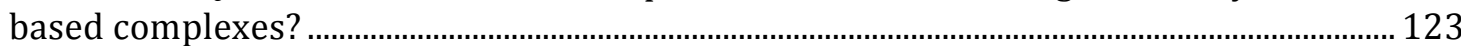

RESEARCH QUESTION 5: Do changes in Nrf2 activity affect regulation of AQP2 in Cul3

conditional knockout or Cul3 4 403-459 mice? ………............................................................... 125

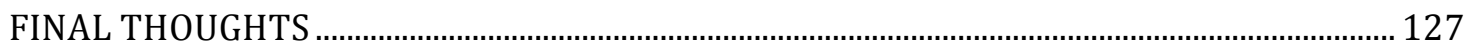

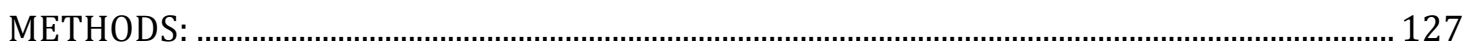

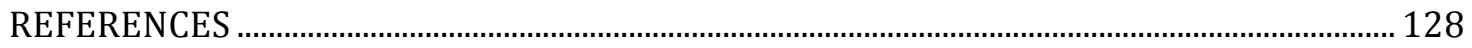

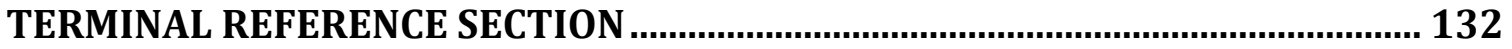




\section{LIST OF TABLES:}

CHAPTER 3:

Table 3.1: Potential binding partners of Klhl3 .............................................. Pg. 76 


\section{LIST OF FIGURES}

\section{CHAPTER 1:}

Figure 1.1: Ubiquitin is a small protein that is attached to substrates. Pg. 4

Figure 1.2: Examples of ubiquitin chains.

Figure 1.3: Ubiquitin attachment is a three-step process. Pg. 6

Figure 1.4: A comparison between Cul1 and Cul3-based complexes. Pg. 8

Figure 1.5: Cyclins and Cdks regulate cell-cycle transitions.

Pg. 12

\section{CHAPTER 2:}

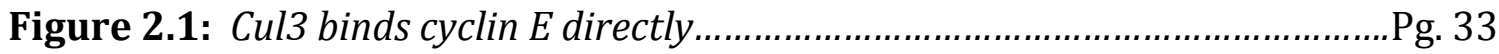

Figure 2.2: Mutants of cyclin E1.

Figure 2.3: The $N$-terminus of cyclin E interacts with Cul3..................................... 36

Figure 2.4: Mutations near the $N$-terminus of cyclin $E$ result in increased stability.....

Pg. 39

Figure 2.5: Mutant localization.

Pg. 41

Figure 2.6: $K 48$ is a Cul3 ubiquitination site on cyclin $E$

Pg. 44

Figure 2.7: The N-terminal domain of cyclin $E$ is required for Cul3-mediated degradation

Figure 2.8: $L M W$ cyclin $E$ is not degraded by Cul3.

Figure 2.9: Cyclin E2 is not a Cul3 substrate

Pg. 48

Figure 2.10: The excess cyclin $E$ in Cul3 KO 293 cells increases proliferation. Pg. 50

Figure 2.11: Cells lacking Cul3 enter S-phase early

Pg. 51

Figure 2.12: Cyclin E levels increase early in cells that are hypomorphic for Cul3

Pg. 51

Figure 2.13: RhoBTB3 interacts with the $N$ terminus of cyclin E........................... 54

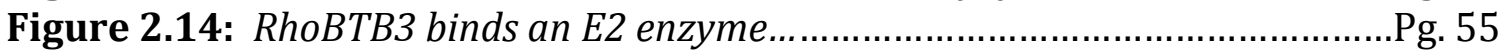

Figure 2.15: Cul3 requires a BTB protein to interact with UbE2E1 ........................ 55

Figure 2.16: LMW cyclin E cannot be degraded by Cul3........................................ 61

Figure 2.17: Cyclin E1 contains a Cul3 degron that is conserved in other mammals

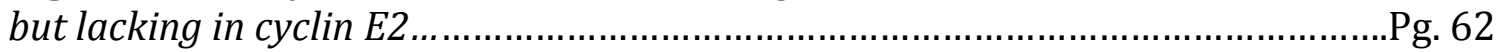

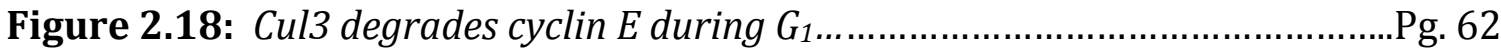

\section{CHAPTER 3:}

Figure 3.1: NCC regulation.............................................................................. 72

Figure 3.2: The human Cul3 mutant localizes to the cytoplasm.......................... Pg. 74

Figure 3.3: Klhl3 structure ......................................................................... Pg. 75

Figure 3.4: P50/dynamitin is a subunit of the dynactin complex........................ Pg. 76

Figure 3.5: P50 and Klhl3 co-localize in the cytoplasm.....................................P. 79

Figure 3.6: P50 interacts with the substrate recognition domain of Klhl3 .......... Pg. 80

Figure 3.7: A human Klhl3 mutant results in p50 stability ..................................... 81

Figure 3.8: P50 binds Cul3 and Cul3 mutants...................................................... 83

Figure 3.9: $P 50$ is ubiquitinated in the presence of Cul3 and Klhl3........................ 84

Figure 3.10: Levels of endogenous $p 50$ are unaffected by loss of Cul3..................Pg. 85 
Figure 3.11: Transfected p50 is more stable in Cul3 KO cells.

.Pg. 86

Figure 3.12: An siRNA targeting Klhl3 effectively decreases the expression of transfected Klhl3 protein

Figure 3.13: Regulation of $A Q P 2$ expression at the membrane

\section{CHAPTER 4:}

Figure 4.1: Cul3 localizes with LRR proteins

Pg. 107

Figure 4.2: Proline-rich regions in BTB proteins.

Figure 4.3: Sequences of MUF1 and cyclin $E$

Figure 4.4: MUF1 binds Cdk2.

Pg. 114

Figure 4.5: Expression levels of transfected MUF1 in the presence of different

proteins.

Pg. 115

Figure 4.6: Model showing two new hypotheses regarding the potential involvement of MUF1 in regulation of Cul3-based complexes. Pg. 116

Figure 4.7: A heavy cyclin E band is present in Cul3 KO 293 cells.

Pg. 121

Figure 4.8: The heavy cyclin $E$ band disappears after incubation in transglutaminase. Pg. 122

Figure 4.9: The effects of transfected transglutaminase on cyclin $E$

Pg. 123 


\section{LIST OF ABBREVIATIONS USED}

APC: Anaphase Promoting Complex

AQP2: Aquaporin 2

BACK: BTB and C-terminal Kelch

BCR: $\mathrm{BTB}, \mathrm{Cul} 3, \mathrm{Rbx} 1$

BTB: Bric-a-brac, Tramtrack, Broad-complex

Cdk: Cyclin-dependent kinase

CHX: Cycloheximide

CKI: Cyclin-dependent kinase inhibitor

Cul1: Cullin 1

Cul2: Cullin 2

Cul3: Cullin 3

Cul4A: Cullin 4A

Cul4B: Cullin 4B

Cul5: Cullin 5

Cul7: Cullin 7

DCT: Distal convoluted tubule

ECM: Extracellular matrix

FHHt: Familial Hyperkalemic Hypertension: Also called PHAII or Gordon's syndrome.

HECT: Homologous to E6-AP Carboxyl Terminus: a class of E3 ubiquitin ligases 
HIF $\alpha$ : Hypoxia-inducible factor alpha: HIF $\alpha$ has been suggested to be a substrate of RhoBTB3.

KO: Knockout

LMW: Low molecular weight

LRR: Leucine-rich repeat

LRR3 (SALM1): LRR-domain protein 3, also called SALM1 (synaptic adhesion like molecule 1)

LRR5 (FMOD): LRR-domain protein 5, also called FMOD (fibromodulin)

MEFs: Mouse embryonic fibroblasts

ORC: Origin recognition complex

PHAII: Pseudohypoaldosteronism type II: Also called FHHt.

PKA: Protein kinase A

Rb: Retinoblastoma protein

ROS: Reactive oxygen species: Oxidative stress

SCF: Skp1, Cul1, F-box

TG2: Transglutaminase 2 (tissue transglutaminase)

WNK1: With no lysine (K) 1: A kinase involved in NCC regulation.

WNK4: With no lysine (K) 4: A kinase involved in NCC regulation.

WT: Wild-type 


\section{GLOSSARY}

Apical membrane: The apical membrane is found in the nephron where it contacts the urine. AQP2 is localized to the apical membrane.

AQP2: Aquaporin 2 is a water channel protein that resides in the apical surface of the collecting duct. AQP2 helps to maintain blood pressure via the reabsorption of water.

ATP1B1: A subunit of the $\mathrm{Na}+\mathrm{K}+$ ATPase. This subunit was identified as a possible binding partner of Klhl3 (Chapter 3).

Basolateral membrane: The basolateral membrane contacts the interstitial fluid in the nephron.

BTB: A domain found in proteins that function as Cul3 substrate adaptors. The BTB domain binds to Cul3.

Cdk: Cyclin-dependent kinases are responsible for cell cycle progression. A Cdk is active when it is bound to a cyclin.

Cdk2: The Cdk binding partner of cyclin E. Cyclin /Cdk2 complexes regulate the $\mathrm{G}_{1} / \mathrm{S}$ transition and release from quiescence.

CKI: Cyclin-dependent kinase inhibitor. There are two families of CKIs: INK4, which inhibit Cdk4/6, and the CIP/KIP family, which includes p21 and p27.

Cre: Cre recombinase is an enzyme that can be used in a conditional knockout mouse model to recombine the DNA that is surrounded by two LoxP sites (floxed). 
Ctb73: A putative substrate adaptor for $\mathrm{Cul3}$, Ctb73 contains an N-terminal BTB domain, a central BACK domain, and a C-terminal PHR domain.

Cul1: One of two E3 ligases responsible for cyclin E ubiquitination. Cul1 uses substrate adaptors with two subunits: The Skp1 linker protein and an F-box protein.

Cul3 4403-459: A Cul3 mutation that results in the skipping of exon 9 of the Cul3 protein. This mutation causes FHHt in humans.

Cul3: An E3 ubiquitin ligase that targets many substrate, including cyclin E, for ubiquitination. Cul3 uses BTB-domain containing proteins as substrate adaptors.

Cul3K712R: Cul3K712R is inactive because it cannot be neddylated.

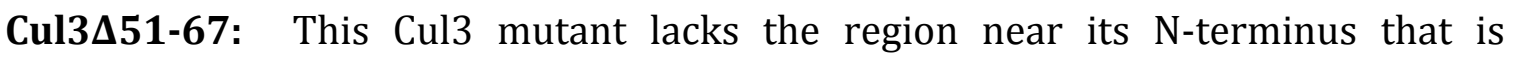
responsible for binding BTB proteins (substrate adaptors).

Cul5: Cul5 is an E3 ligase that uses B/C box or VHL proteins and substrate adaptors.

Cyclin E: Cyclin E controls the $\mathrm{G}_{1} / \mathrm{S}$ transition by binding and activating Cdk2. Cyclin E/Cdk2 complexes phosphorylate themselves in addition to many other substrates including the $\mathrm{Rb}$ protein. Eukaryotes contain two cyclin $\mathrm{E}$ proteins, cyclin E1 and cyclin E2, which are products of two different genes.

Cyclin homology domain: This domain is responsible for cyclin E's ability to bind Cdk2 and is found in the central part of cyclin E's structure.

Cyclins: A class of proteins responsible for binding and activating Cdks, resulting in cell cycle progression. 
Dynamitin/p50: A subunit of the dynactin complex. This complex is responsible for endosomal transport. P50 links the microtubule binding and cargo binding portions of the complex. P50 was identified as a Klhl3 interacting protein and possible substrate (Chapter 3).

E1 ubiquitin activating enzyme: E1 enzymes are responsible for activating the Cterminal Gly-Gly motif on ubiquitin and preparing it for attachment to a substrate. Activation of ubiquitin by an E1 occurs via an ATP-dependent reaction.

E2 ubiquitin conjugating enzyme: E2 enzymes work with E3 ubiquitin ligases in order to transfer ubiquitin to substrates.

E2F: A transcription factor involved in cell cycle regulation. E2F is sequestered by the $\mathrm{Rb}$ protein, which releases E2F upon sufficient phosphorylation by cyclins and Cdks. Once E2F is free of Rb, it is able to activate transcription, resulting in entry into S-phase.

E3 ubiquitin ligase: E3 ligases provide substrate specificity to the ubiquitin system. Cullin E3s work with an E2 to facilitate ubiquitin attachment. HECT E3 ligases and cullin ligases represent two classes of E3s.

EEA1: A marker of early endosomes.

ENaC: A sodium channel located in the nephron.

F-box: Cul1 utilizes substrate adaptors, such as Fbxw7, that contain F-box domains to recognize substrates.

FAZF: A BTB protein that also contains a zinc-finger domain. FAZF binds LRR5 (FMOD) (Chapter 4). 
Fbxw7: An F-box protein that functions as a substrate adaptor for Cul1. Fbxw7 is involved in Cul1-mediated ubiquitination of cyclin E.

FHHt: Familial Hyperkalemic Hypertension: Also called PHAII or Gordon's syndrome. Mutations in Cul3 and Klhl3 cause this disease.

Floxed: "Flanked by LoxP": This is a DNA construct that uses LoxP sites to make conditionally -expressing mouse alleles. Cre recombinase can be expressed in mice that carry a floxed allele, resulting in the deletion of the selected DNA. The Cre LoxP system is useful for studying essential genes, as it allows for tissue specific deletion.

$G_{0}$ : $G_{0}$ is another name for the quiescent stage of the cell cycle. A cell arrested in quiescence is not preparing to divide, but can re-enter the proliferative cell cycle if given the appropriate mitogenic signals.

$\mathbf{G}_{\mathbf{1}}$ : This is the first growth phase that takes place during interphase of the cell cycle. $\mathrm{G}_{1}$ occurs prior to S-phase.

$\mathbf{G}_{2}: \mathrm{G}_{2}$ is the growth phase following DNA synthesis but prior to mitosis.

Gli: A family of mammalian transcription factors that are involved in the hedgehog signaling pathway. The Gli proteins are homologues of Ci in Drosophila. Gli proteins, as well as $\mathrm{Ci}$, are ubiquitinated by $\mathrm{Cul} 3$.

GSK3: A kinase that phosphorylates cyclin E.

Interphase: Interphase consists of the $\mathrm{G}_{0}, \mathrm{G}_{1}, \mathrm{~S}$, and $\mathrm{G}_{2}$ phases of the cell cycle.

KCTD13: KCTD13 is the BTB substrate adaptor that targets RhoA for Cul3mediated degradation. 
Keap1: A prominent member of the BTB-BACK-Kelch family of proteins, Keap1 targets the transcription factor Nrf2 for degradation.

Kelch domain: A common substrate-recognition domain that is found in many BTB-domain containing proteins. Some notable members of this family are Keap1, which regulates Nrf2, and Klhl3, which is involved in blood pressure regulation.

Klhl17 (actinfilin): A BTB-BACK-Kelch protein that is thought to be a Cul3 substrate adaptor.

Klhl2 (Mayven): A BTB-BACK-Kelch protein and Cul3 substrate adaptor, Klhl2 is closely related to Klhl3.

Klhl24: Klhl24 is a BTB-BACK-Kelch protein and Cul3 substrate adaptor that ubiquitinates keratin 14 .

Klhl3: A BTB-BACK-Kelch protein that has been shown to cause FHHt in humans when mutated.

Klhl3R528H: A dominant mutation in Klhl3 that has been associated with FHHt.

LAMP-1: A marker that can be used to identity late endosomes.

MATH domain: A secondary domain that is found in the Cul3 substrate adaptor SPOP.

MEI-1: A katanin protein involved in microtubule dynamics, MEI-1 is ubiquitinated by Cul3 and MEL-26.

MEL-26: A BTB protein that works with Cul3 to ubiquitinate MEI-1.

MG132: A proteasome inhibitor that when added to cultured cells, results in increased stability of some ubiquitin-proteasome system substrates. xiv 
Mitosis: The final portion of the proliferative cell cycle when a cell completes cell division, resulting in two daughter cells.

MSI2: A substrate of the Cul3 ${ }^{\text {RhoBTB2 }}$ ligase that is associated with breast cancer.

MUF1 (LRRC41): An LRR-domain containing protein that also contains a B/C/ box, making it a possible Cul5 substrate adaptor. MUF1 can bind Cdk2 (Chapter 4).

$\mathrm{Na}+\mathbf{K}+$ ATPase: This transporter is found in a variety of tissues and is responsible for creating an ion gradient in the cells of the nephron. The beta subunit of this transporter was identified as a potential Klhl3-interacting protein (Chapter 3).

NCC transporter: NCC is a sodium chloride transporter found in the DCT of the nephron. Improper regulation of NCC causes FHHt hypertension.

Nedd8: Nedd8 is a ubiquitin-like molecule involved in Cul3 function. Cul3 is modified by Nedd8 on K712.

Nrf2: Nrf2 is a transcription factor involved in the stress-response. Nrf2 is a substrate of the Keap1 BTB-Kelch protein and Cul3.

OSR1: OSR1 is a kinase that activates NCC via phosphorylation.

PEST: A short sequence that was identified in proteins with high turnover. Cyclin E has a PEST sequence located near its C-terminus.

PHR domain: A secondary domain that is similar to a kelch domain. Several BTBproteins also contain PHR domains. Kelch and PHR domains are involved in Cul3 substrate recognition.

PLZF: A zinc-finger domain containing BTB protein. PLZF is closely related to FAZF. 
Rb: The retinoblastoma protein plays a crucial role in cell cycle progression, as it is responsible for binding and sequestering the E2F transcription factor, which is necessary for S-phase to begin. Sufficient phosphorylation of Rb by cyclin/Cdk pairs results in the release of E2F, allowing for transcription of Sphase associated genes to begin.

Rbx1: The "ring-finger" protein, which interacts with cullin ubiquitin E3 ligases near their C-termini. Rbx1 is believed to play a role in E2 binding.

RCBTB1: RCBTB1 is a BTB protein that has been associated with the eye disease retinitis pigmentosa.

RhoA: RhoA is a small GTPase that regulates many processes, including the actin cytoskeleton. RhoA is ubiquitinated and degraded in a Cul3 ${ }^{\text {KCTD13 dependent }}$ manner.

RhoBTB2: A member of the RhoBTB protein family, RhoBTB2 targets MSI2 for degradation.

RhoBTB3: A member of the RhoBTB protein family that binds cyclin E. Others have suggested that RhoBTB3 and other members of the RhoBTB family associate with MUF1.

S-phase: The cell cycle stage during which a cell replicates its DNA.

Skp1: Skp1 forms part of Cul1-based complexes as it is responsible for linking Cul1 with an F-box protein.

SPAK: SPAK is a kinase that regulates NCC. See OSR1.

SPOP: Also called Ctb75, SPOP is a Cul3 substrate adaptor (BTB protein). 
Substrate adaptor: Substrate adaptors are proteins that bind to cullin-based E3 ligases in order to recognize specific substrates for ubiquitination. Each cullin ligase can associate with many different substrate adaptors, allowing each cullin to have many substrates.

UbE2E1: UbE2E1 is an E2 ubiquitin conjugating enzyme, which forms degradative (K48) ubiquitin linkages. UbE2E1 binds RhoBTB3 (Chapter 2).

Ubiquitin: Ubiquitin is a small protein that is attached to target proteins, often resulting in their degradation.

WNK1: With no lysine (K) 1: A kinase involved in NCC regulation. WNK1 can bind and be ubiquitinated by Klhl3.

WNK4: With no lysine (K) 4: A kinase involved in NCC regulation. WNK4 interacts with and can be ubiquitinated by Klhl3.

Zinc Finger domain: A DNA-binding domain that is present in some BTB proteins, for example PLZF and FAZF. 
CHAPTER 1: Introduction and Background 


\section{THE CUL3 UBIQUITIN LIGASE}

Post-translational modifications of proteins such as phosphorylation, methylation, acetylation, and ubiquitination are essential for a wide variety of cell signaling events. The ubiquitin system is one crucial method of cellular signaling in eukaryotic cells in which a small protein called ubiquitin (Figure 1.1) is covalently attached to lysine residues on target proteins, also called substrates (Metzger et al. 2012). Substrates can be either monoubiquitinated (one molecule of ubiquitin is attached) or polyubiquitinated with a ubiquitin chain consisting of multiple ubiquitin molecules (Hicke 2001). Histones are often modified post-translationally by monoubiquitination (Cole et al. 2015). Ubiquitin chains can form different conformations (Grice and Nathan 2016), which are determined by the specific lysine residues on the ubiquitin molecules that comprise the chain (Figure 1.2). Ubiquitinated proteins can be differentiated by the cell depending upon the type and length of ubiquitin chain that is attached. For example, K48-linked ubiquitin chains signal the ubiquitinated protein to be degraded by the proteasome whereas K63-linked chains are involved in different processes (Lim and Lim 2011). The attachment of ubiquitin signals a target protein to undergo a variety of cellular processes including degradation by the $26 \mathrm{~S}$ proteasome (Grice and Nathan 2016). The ubiquitin system relies upon a complex set of biochemical mechanisms allowing for proper substrate identification and ubiquitin attachment. Ubiquitin signaling is essential for the survival and proper function of eukaryotic cells (Finley et al. 1987). 
The transfer of ubiquitin to a substrate requires three enzymes (Figure 1.3). First, an E1, also called an ubiquitin activating enzyme, attaches the C-terminal Gly residue of ubiquitin to its active site in an ATP-dependent reaction (Haas et al. 1982). Next, The E1 transfers the ubiquitin to the active site of an E2 ubiquitinconjugating enzyme. Lastly, the E2 will work with an E3 ubiquitin ligase to form an isopeptide bond attaching the ubiquitin (via the C-terminal Gly residue) to a lysine residue on the target protein (Haas and Rose 1982; Pickart and Rose 1985). Ubiquitin ligases are a diverse class of molecules, which is important because they are responsible for providing substrate specificity to this system (Gonen et al. 1996). Thus, ubiquitin ligases ensure that the appropriate target protein is ubiquitinated at the proper time. 


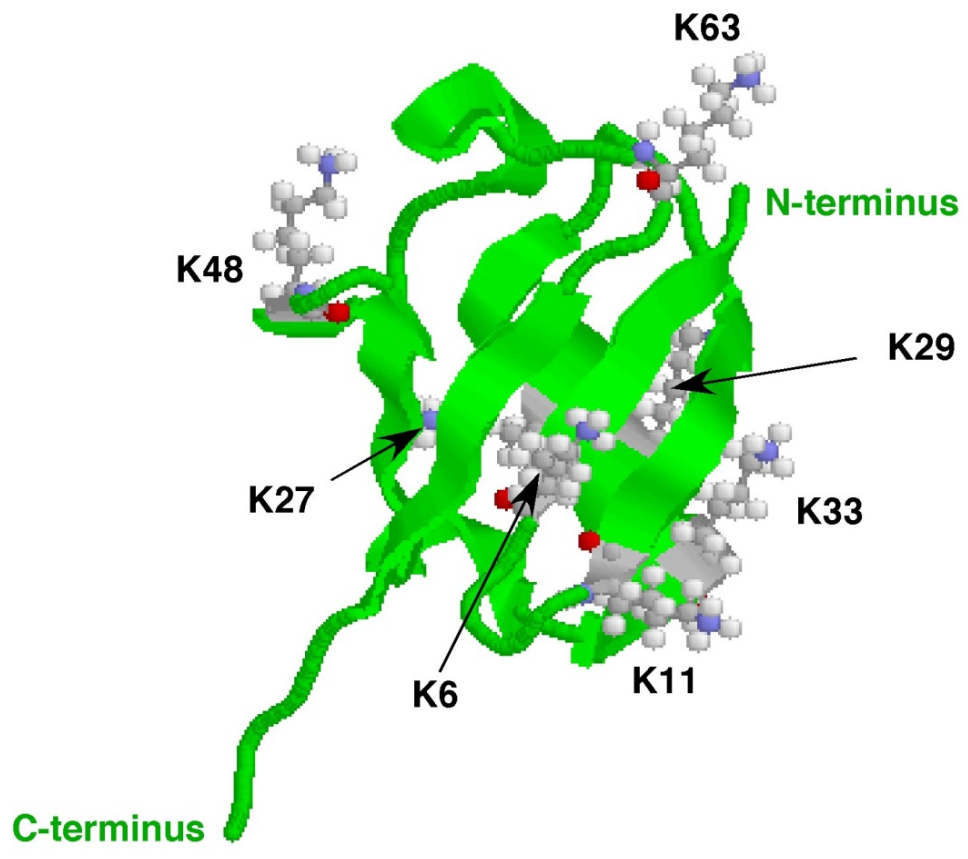

Figure 1.1: Ubiquitin is a small protein that is attached to substrates. Ubiquitin is a small protein, the C-terminus of which is attached to substrates and other ubiquitin molecules via lysine residues. Different conformations of chains are formed depending upon which ubiquitin lysines are involved; for example K48-linked chains target substrates for proteasomal degradation. 


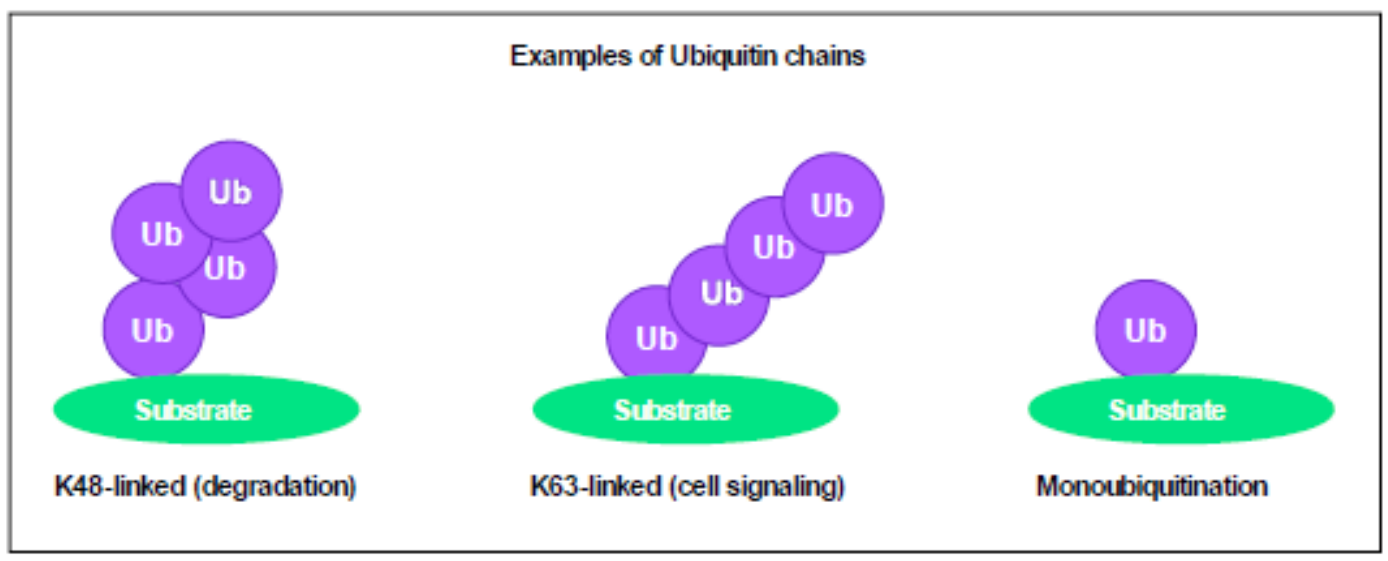

Figure 1.2: Examples of ubiquitin chains. K48-linked chains have a globular shape and are associated with degradation (Left). K63-linked chains have a linear structure and are associated will non-degradative processes (Center). A substrate can also be monoubiquitinated, which is necessary for regulation of histone proteins (Right). 


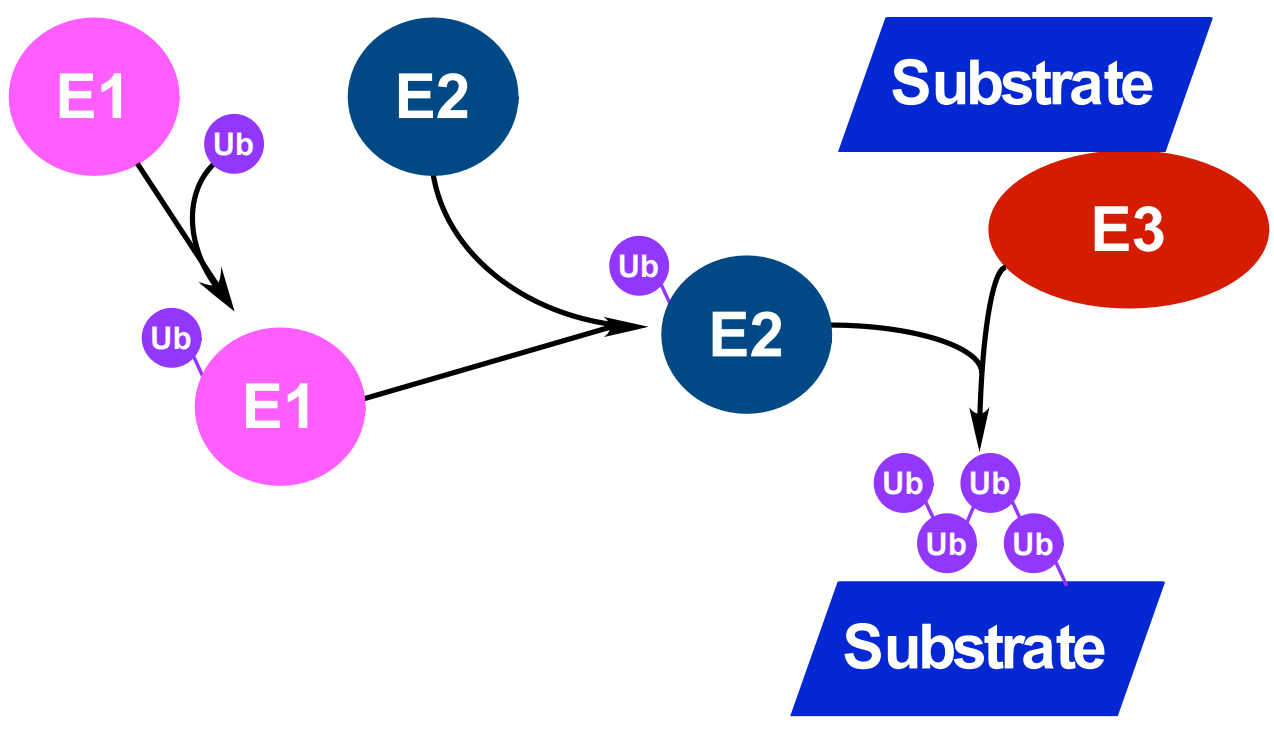

Figure 1.3: Ubiquitin attachment is a three-step process. Attachment of ubiquitin to a substrate requires three events: First, an E1 activating enzyme prepares the ubiquitin molecule for conjugation. Next, the E1 transfers the ubiquitin to the active site Cysteine on the E2 ubiquitin-conjugating enzyme. Lastly, the E2 will work with an E3 ubiquitin ligase, which provides substrate specificity, to attach the ubiquitin to the substrate.

There are two main classes of E3 ubiquitin ligases. The first class is called the HECT (homologous to E6-AP carboxyl terminus) ubiquitin ligases, which can directly catalyze the transfer of an activated ubiquitin molecule to a substrate (Bernassola et al. 2008). The second class of E3 ligases is called the cullin-RING (really interesting new gene) E3 ligases (Petroski and Deshaies 2005). Unlike the HECT E3s, these cullin scaffolds do not themselves catalyze ubiquitin transfer, but 
instead function to position the substrate in close proximity to the E2, allowing for the attachment of the ubiquitin molecule (Petroski and Deshaies 2005).

Humans possess eight cullin proteins (Cul1, Cul2, Cul3, Cul4A, Cul4B, Cul5, Cul7, and the APC) each of which forms its own set of E3 ligases complexes (Petroski and Deshaies 2005). The cullin-based ligases are divided into several subtypes including Cul1-based (SCF), Cul2-based, Cul3-based etc. The complexes formed by these different ligases have many structural similarities, but each type of ligase is unique and requires a different set of substrate recognition proteins, for example Cul1 uses Skp1 and an F-box protein to recognize substrates, and Cul3 uses BTBdomain containing proteins to bind substrates (Geyer et al. 2003; Petroski and Deshaies 2005). The requirement of a second protein, or substrate adaptor, to bind a substrate, allows for each cullin to have a diverse set of substrates and affect a wide variety of cellular processes. Cul1 and Cul3 have both been shown to degrade

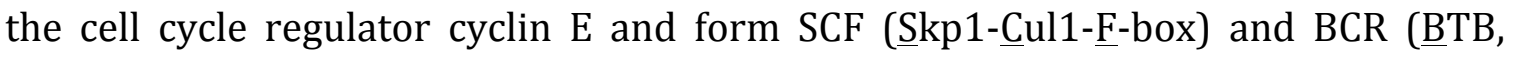
Cul3, Rbx1) complexes respectively (Clurman et al. 1996; Singer et al. 1999; Petroski and Deshaies 2005). Cul1-based complexes have been extensively studied and the structure of these complexes is well known (Figure 1.4). The structure of Cul3based complexes is not as well understood, even though they are crucial for cellular functioning and Cul3 is an essential gene in mice (Singer et al. 1999). 


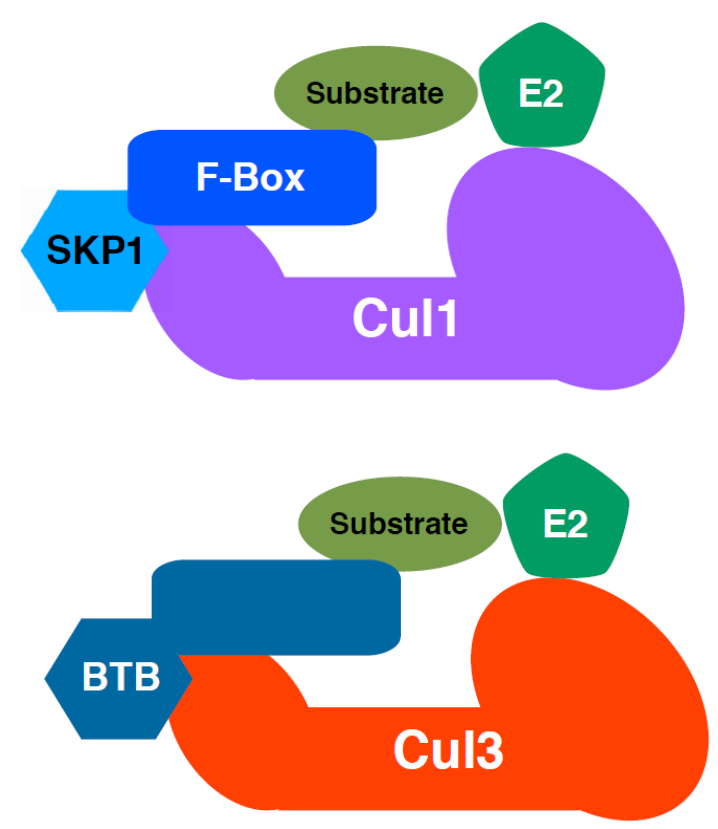

Figure 1.4: A comparison between Cul1 and Cul3-based complexes. Cul1 (SCF, top, purple) uses a two-subunit substrate adaptor consisting of Skp1 and an F-box protein whereas Cul3 (BCR, bottom, red) relies upon one-subunit substrate adaptors called BTB proteins, which contain both a Cul3 binding domain and a substrate interaction domain. Both Cul1 and Cul3 bind the ring-finger protein, $\mathrm{Rbx1}$, and associate with E2 enzymes (green) via the C-terminal domain of the cullin.

Both Cul1 and Cul3 possess an N-terminal domain which interacts with substrate adaptors, a central cullin homology domain, and a C-terminal region that is modified by the ubiquitin-like protein Nedd8, interacts with the ring finger protein Rbx1, and interacts with the E2 enzyme (Hori et al. 1999; Zheng et al. 2002; Wimuttisuk and Singer 2007). Current understanding of cullin architecture posits that the $\mathrm{E} 2$ binds to Rbx1, which is located near the C-terminus of the cullin (Kleiger et al. 2009; Metzger et al. 2014). For example, it has been shown that the acidic tail on the E2 enzyme Cdc34 interacts with a basic region near the Rbx1 binding site on Cul1 (Kleiger et al. 2009). SCF modification of Cul1 by Nedd8 increases SCF activity 
and may be involved in recruiting the $\mathrm{E} 2$ to the complex, demonstrating the importance of Nedd8 modification for cullin functioning (Kawakami et al. 2001).

In order to recognize substrates, Cul1 relies upon substrate adaptors comprised of Skp1 and an F-box protein. Skp1 helps to associate the F-box protein with the Cul1 complex, whereas the F-box protein is responsible for recognizing and recruiting phosphorylated substrates to the complex (Skowyra et al. 1997; Zheng et al. 2002).

Cul3 relies upon a class of proteins containing BTB (Bric-a-brac, Tram track, Broad Complex) domains to recognize substrates (Xu et al. 2003; Pintard et al. 2004). These proteins contain one or more BTB domains that interact with Cul3, as well as a secondary domain such as a Kelch domain or MATH domain which is responsible for substrate binding (Stogios et al. 2005). BTB proteins work with Cul3 to regulate a variety of cellular processes. The BTB family of proteins has many members including MEL-26 which ubiquitinates the katanin protein MEI-1, RhoBTB3 which is believed to ubiquitinate both cyclin E and $\mathrm{HIF} \alpha$, Klhl3 which is involved in blood pressure regulation by ubiquitinating WNK4, and Klhdc5 (Ctb9) which ubiquitinates the microtubule-severing protein p60/katanin (Pintard et al. 2003; Cummings et al. 2009; Johnson et al. 2009; Lu and Pfeffer 2013; Shibata et al. 2013; Wakabayashi et al. 2013; Zhang et al. 2015). Coordinated ubiquitination of a substrate by more than one ligase is known to occur. For example, the cell-cycle regulator Cyclin E provides us with an example of a substrate that is degraded by two different cullin-based E3 ligases, Cul1 and Cul3 (Clurman et al. 1996; Singer et 
al. 1999). The ubiquitination of the Gli proteins in the hedgehog-signaling pathway is also known to occur via both Cul1 and Cul3-dependent mechanisms (Jiang 2006).

\section{CELL CYCLE REGULATION: Basic mechanisms controlling proliferation}

The cell division cycle is one crucial process in which the ubiquitin system has long been known to play an essential role. Interphase of the eukaryotic cell cycle is divided into three stages. The first stage is a growth phase called $\mathrm{G}_{1}$ (Ohtsubo and Roberts 1993). $\mathrm{G}_{1}$ is followed by $\mathrm{S}$ phase during which the DNA is replicated, which in turn is followed by $\mathrm{G}_{2}$. The proliferative cell cycle is completed with "M phase", the phase during which mitosis occurs (Pardee 1974). Eukaryotes have evolved complex mechanisms to ensure that cells replicate their DNA and divide only when appropriate. Mechanisms to ensure the proper replication of DNA are in place throughout interphase of the cell cycle, which helps to ensure that the genome is copied only once and only during S phase (Nishitani and Lygerou 2002). During S-phase, DNA replication begins from pre-determined locations on the DNA, known as origins, which must be properly labeled for replication to occur (Ford and Chevalier 1995). Origins are labeled either late in M-phase or early in G1 (Ford and Chevalier 1995). The labeling of origins during this brief temporal window is one way to decrease the chance that re-replication will occur, as it prevents the labeling of more origins later on during the cell cycle (Ford and Chevalier 1995). Following the labeling of an origin during $\mathrm{G}_{1}$, the proteins $\mathrm{Cdt} 1$ and $\mathrm{Cdc} 6$ will associate with the ORC (origin recognition complex). The next step in the replication process is called licensing, which can only occur during $G_{1}$ (Nishitani and Lygerou 2002). 
During this step, two proteins, Cdt1 and Cdc6, load the replication machinery onto the origin in preparation for DNA replication (Nishitani and Lygerou 2002).

The cell cycle also has a special state referred to as ' $\mathrm{G}_{0}$ ' or 'quiescence', during which the cell is not preparing to divide. Unlike senescent cells, which can no longer divide, quiescent cells possess the ability to exit the $G_{0}$ state and reenter the cell cycle if given the proper molecular signals (Siddiqi and Sussman 2014). The quiescent state of the cell cycle is imperative to the normal functioning of eukaryotic organisms. At any given time, a large portion of the cells in a multicellular organism are arrested in this quiescent state. Quiescence is actively regulated by the cell and is necessary to prevent excessive proliferation and preserve the integrity of the cell's DNA (Cheung and Rando 2013). Abnormal release from quiescence can results in unwanted proliferation and can be harmful to the organism. Intricate processes regulate the cell cycle during all its stages and are governed by cyclin/Cdk (cyclin dependent kinase) protein complexes. These complexes drive the cell cycle forward via the phosphorylation of a wide variety of substrates.

\section{CELL CYCLE REGULATION: Cyclins and Cdks}

Cyclins are essential for proper cell cycle control. Mammalian cells have several different cyclins, and each one is active at a specific time point during the cell cycle (Figure 1.5). Therefore, the level of any given cyclin does not remain constant, but instead rises and falls throughout the different stages of the cell cycle. Proper temporal regulation of cyclin activity throughout the cell cycle is necessary for proper functioning of the cell and the health of the organism as a whole. 


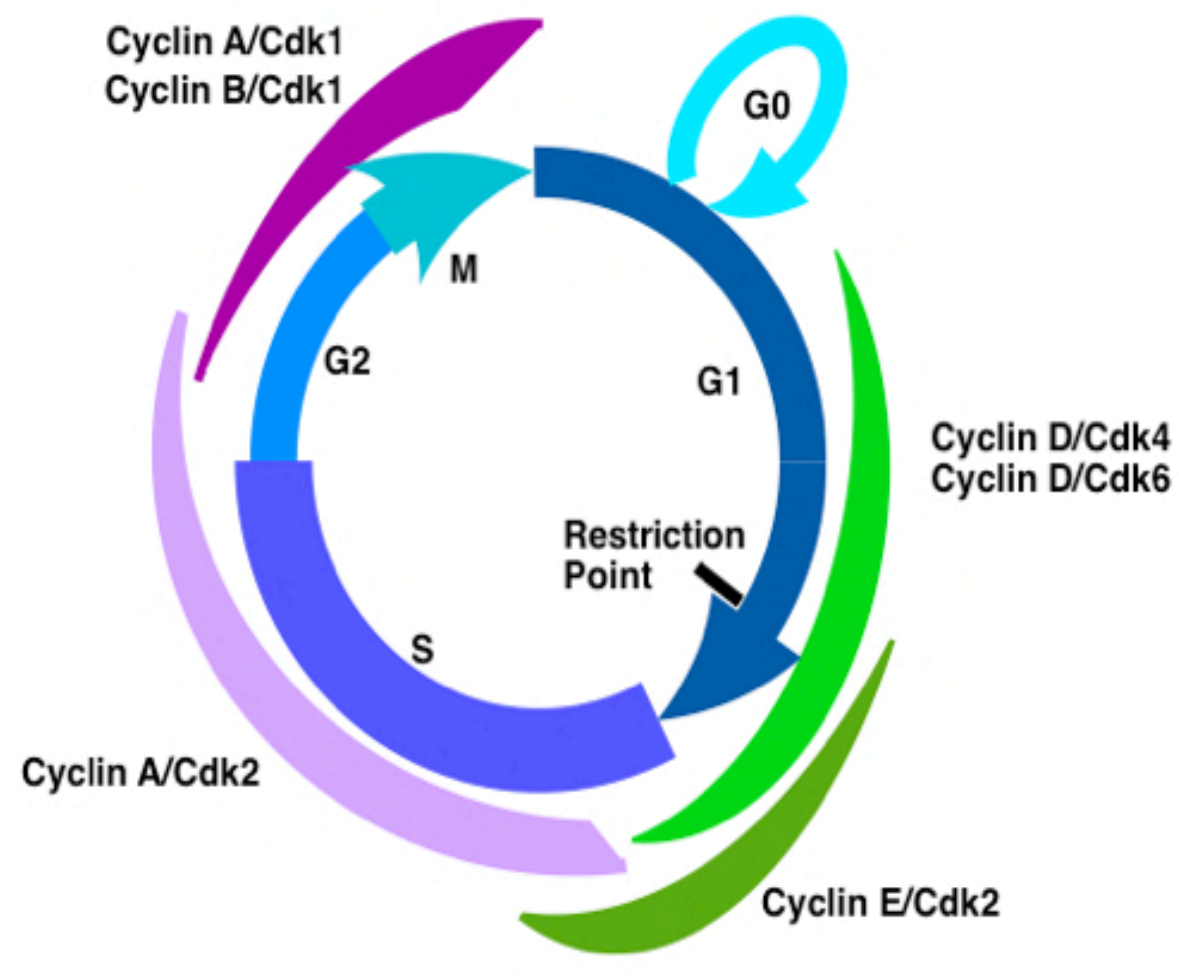

Figure 1.5: Cyclins and Cdks regulate cell-cycle transitions. Cyclin D and its partner Cdk4/6 are responsible for internalizing an external mitogenic signal. An external stimulus causes cyclin $\mathrm{D} / \mathrm{Cdk}$ 4/6 to phosphorylate target proteins, such as $\mathrm{Rb}$, that result in cell cycle progression and increase cyclin E levels. Cyclin E/Cdk2 complexes phosphorylate substrates during the G1/S transition resulting in the start of S-phase. A cell has passed the restriction point once it no longer requires external signals for cell cycle progression to occur, and cyclins $\mathrm{D}$ and $\mathrm{E}$ are involved in this process. Cyclin A/Cdk2 controls progression through S-phase and cyclins A and $\mathrm{B}$ bind Cdk1, controlling progression through G2 and mitosis.

Cyclins bind to and activate enzymes known as Cyclin Dependent Kinases (Cdks) (Koff et al. 1991). When bound to a Cdk, the Cyclin/Cdk complex phosphorylates target proteins that are responsible for cell cycle progression (Koff et al. 1992). Cyclin E is a mammalian cyclin that binds to Cdk2. The Cyclin E/Cdk2 complex is responsible for the phosphorylation of target proteins during the $\mathrm{G}_{1} / \mathrm{S}$ 
transition of the cell cycle (Figure 1.5). The cyclin E gene is one of many cell cycle related genes that is regulated by the E2F transcription factor (Swiss and Casaccia 2010). E2F binds to the $\mathrm{Rb}$ (retinoblastoma) pocket protein, which prevents it from activating transcription (Swiss and Casaccia 2010). Sufficient phosphorylation of Rb by cyclin $\mathrm{D}$ and Cdk4/6 upon mitogen stimulation during $\mathrm{G}_{1}$ of the cell cycle results in release of $\mathrm{E} 2 \mathrm{~F}$ by $\mathrm{Rb}$ and increased expression of E2F controlled genes, including cyclin E (Resnitzky et al. 1994; Sherr 1994; Ekholm and Reed 2000). Cyclin E then binds and activates $\mathrm{Cdk} 2$, resulting in the phosphorylation of $\mathrm{Rb}$ as well as other substrates (Koff et al. 1991; Ohtsubo and Roberts 1993). Cyclin E/Cdk2 substrates include $\mathrm{Rb}$, Cdh1, cyclin E/Cdk2 autophosphorylation, and others (Won and Reed 1996; Abbas et al. 2007; Keck et al. 2007). One essential substrate of cyclin E/Cdk2 is Cdc6, which when stabilized upon phosphorylation by cyclin E/Cdk2, allows for the formation of the pre-replication complex prior to the start of S-phase (Ayad 2005; Mailand and Diffley 2005).

$\mathrm{G}_{1}$ contains a point known as the "Restriction point" at which time the cell has committed to duplicating its DNA and will continue on into S-phase even if the external mitogenic stimuli are removed (Pardee 1974; Blagosklonny and Pardee 2002). Cyclin/Cdk activity contributes to the cell's ability to reach this point (Blagosklonny and Pardee 2002). This mechanism plays an important role in both the entry into S-phase and release from quiescence.

Eukaryotes contain two cyclin E proteins, cyclin E1 and cyclin E2, which are products of two different genes, CCNE1 and CCNE2 (Sherr and Roberts 1999; Geng 
et al. 2003; Perez-Neut et al. 2015). Both cyclin E proteins bind and activate Cdk2 (Perez-Neut et al. 2015). Cyclin E is significant as its overexpression is associated with increased proliferation and release from quiescence as well as aneuploidy, polyploidy, and delayed progression through mitosis (Spruck et al. 1999; Keck et al. 2007). Overexpression of cyclin E in cancer cells is associated with increased tumorigenesis and poor clinical prognosis (Said and Medina 1995) . In contrast, mouse fibroblasts that lack both cyclin E genes, cyclin E1 and cyclin E2, are unable to exit from the quiescent state but loss of cyclin E has little effect on cells that are already proliferating (Geng et al. 2003).

As cyclin E plays a significant role in cell cycle progression, it stands to reason that it is a highly modified, highly regulated protein. Cyclin E1 and cyclin E2 are regulated by different mechanisms (Perez-Neut et al. 2015). The remainder of this section discusses regulation of cyclin E1. Cyclin E1 is regulated via phosphorylation, both auto phosphorylation when bound to Cdk2 and phosphorylation by different kinases such as GSK3 (Clurman et al. 1996; Welcker et al. 2003). Cyclin $\mathrm{E}$ is also regulated in a ubiquitin-dependent manner, resulting in its degradation by the 26S proteasome (Clurman et al. 1996). Lastly, activity of cyclins and Cdks is regulated via a class of proteins called cyclin dependent kinase inhibitors or CKIs (Besson et al. 2008). There are two types of CKIs: The first type includes the INK4 proteins, which exclusively regulate Cdks 4 and 6 (Sherr and Roberts 1999). The second class, which regulates cyclin E and Cdk2 in addition to others, includes the proteins p27, p21, and p57 (Sherr and Roberts 1999). Proteins 
like p21 and p27 inhibit cyclin/Cdk activity by binding to various cyclins and Cdks (Sherr and Roberts 1999).

Additionally, research has demonstrated that in several cancers, including breast, ovarian, melanoma, and others, cyclin E1 can be proteolytically cleaved resulting in truncated low molecular weight (LMW) cyclin E proteins, which mainly lack their N-termini. These LMW cyclin E are able to activate Cdk2 variants are associated with tumorigenesis and poor prognosis in cancer patients (Said and Medina 1995; Harwell et al. 2000; Porter et al. 2001).

\section{CELL CYCLE REGULATION: Mechanisms controlling cyclin E abundance}

Cells maintain proper levels of Cyclin E through a combination of production via 1) tight transcriptional regulation, ensuring that it is made only during the correct temporal window and 2) degradation (Clurman et al. 1996; Singer et al. 1999). This degradation is also stringently regulated and is frequently initiated by the addition of ubiquitin. The covalent attachment of ubiquitin to cyclin E results in degradation by the $26 \mathrm{~S}$ proteasome.

Two ubiquitin ligase pathways are responsible for signaling the degradation of Cyclin E. The first is the Cul1 (Cullin1) ubiquitin ligase, which functions as part of a complex that recognizes a phosphorylated Threonine residue (T395 in humans, T393 in mice) on Cyclin E (Clurman et al. 1996; Koepp et al. 2001; Strohmaier et al. 2001; Loeb et al. 2005). After recognizing Cyclin E, the Cul1 complex can ubiquitinate it for degradation by the proteasome. In addition to Cul1, the E3 ligase Cullin3 (Cul3) also functions in the degradation of cyclin E when it is not bound to 
Cdk2 (Singer et al. 1999). The mechanism by which Cul3 degrades cyclin E is not well understood. Previous work from our lab which utilized a condition knockout of Cul3 has shown that Cul3 is necessary for the degradation of cyclin $\mathrm{E}$ and maintenance of quiescence in the murine liver (McEvoy et al. 2007).

\section{CUL3 AND DISEASE: Cancer}

Cul3 is an essential gene, as ablation of Cul3 in mice resulted in embryonic lethality (Singer et al. 1999). As Cul3 is necessary for cellular functioning, it is not surprising to learn that Cul3 has been associated with several human diseases, including cancer (Genschik et al. 2013). Cancer is a complicated disease involving many cellular and physiological pathways including those involved in cell division and responses to stress. Cancer develops when a cell undergoes DNA mutation which results in transformative changes involving a variety of cellular pathways that are meant to control proliferation, resulting in a cancerous cell that can grow and metastasize (Penkert et al. 2016). Determining the exact role(s) Cul3 plays in cancer development poses a difficult task as Cul3 regulates dozens of substrates, many of which remain unknown. Here, some of the possible roles of Cul3 in cancer are summarized.

One well-studied substrate of the Cul3 ligase is Nrf2, a transcription factor involved in the oxidative stress response, which is a substrate of Cul3 and the Keap1 BTB protein (Itoh et al. 1999; Cullinan et al. 2004; Kobayashi et al. 2004; Zhang et al. 2004; Furukawa and Xiong 2005). The involvement of the Nrf2-Keap1-Cul3 pathway in many diseases including cancer has been extensively reviewed 
(Leinonen et al. 2015; Holmstrom et al. 2016; Kim and Keum 2016; Sparaneo et al. 2016). In short, the BTB protein Keap1 and other mechanisms tightly regulate Nrf2 when the cell is not under stress, but upon stimulation by stress induced by ROS (reactive oxygen species), a structural modification of Keap1 occurs which reduces degradation of Nrf2 allowing it to activate transcription of Nrf2-controlled genes (Harder et al. 2015). Nrf2 plays a complicated role in cancer as Nrf2 helps to prevent the development of cancer in healthy cells, but some cancerous cells also have elevated Nrf2 activity which is associated with resistance to chemotherapy (Harder et al. 2015). Nrf2 may be the most studied Cul3 substrate, and it alone provides Cul3 with a definitive role in cancer biology.

Another well-studied Cul3 substrate involved in cancer is the cell cycle regulator cyclin E. Like Nrf2, cyclin E levels are tightly maintained by Cul3 and other mechanisms in most cells (Singer et al. 1999; McEvoy et al. 2007). Overexpression of cyclin $\mathrm{E}$ is associated with tumorigenesis and truncated cyclin $\mathrm{E}$ variants are also associated with tumorigenesis and cancer (Scuderi et al. 1996; Porter and Keyomarsi 2000; Porter et al. 2001). The regulation of cyclin E by Cul3 will be further analyzed in chapter two. There are many other processes that are regulated by Cul3 that also have demonstrated involvement in cancer. For instance, it has recently been revealed that the substrate adaptor RhoBTB2 is responsible for degrading the oncogenic protein MSI2 in breast cancer (Choi et al. 2016), and mutations in the BTB protein SPOP have been demonstrated in prostate cancer (Mani 2014). The involvement of Cul3 and BTB proteins in cancer has been recently reviewed (Genschik et al. 2013; Chen and Chen 2016). 


\section{CUL3 AND DISEASE: FHHt hypertension:}

Familial hyperkalemic hypertension (FHHt), also known as Gordon's syndrome or PHAII, is an inherited form of hypertension also characterized by hyperkalemia that can be treated with thiazide diuretics (O'Shaughnessy 2015). Genetic studies have identified mutations in two components of a ubiquitin ligase complex, the cullin scaffold protein Cul3, and the BTB domain containing substrate adaptor protein Klhl3, that cause this disease (Boyden et al. 2012; Louis-Dit-Picard et al. 2012). Mutations in the kinases WNK1 and WNK4 were previously known to cause FHHt, which is known to result from misregulation of the NCC sodium chloride cotransporter located in the distal convoluted tubule (DCT) of the nephron of the kidney (O'Shaughnessy 2015).

WNK1 and WNK4 are kinases that are involved in regulation of NCC. The WNK kinases regulate the activity of NCC and its expression at the membrane surface by phosphorylating substrates including the SPAK kinase, which is responsible for directly phosphorylating NCC (Yang et al. 2003; Hoorn and Ellison 2012; Chavez-Canales et al. 2014). Recently, WNK4 has been identified as a substrate of the ubiquitin ligase complex formed by Cul3 and Klhl3 (Ohta et al. 2013; Shibata et al. 2013; Wakabayashi et al. 2013). Degradation of WNK4 by the Cul3 ${ }^{\text {Klhl3 }}$ (nomenclature: Cul3 scaffold containing the Klhl3 BTB protein) ubiquitin ligase complex can partially explain the role of Cul3 in NCC regulation, as WNK4 is necessary for regulation of SPAK and also NCC degradation by the lysosome (Golbang et al. 2006; Hoorn and Ellison 2012). However, patients with Cul3 mutations have a more severe disease phenotype and develop hypertension at an 
earlier age in comparison to patients with mutations in Klhl3, WNK4, or WNK1(Boyden et al. 2012). This led to the hypothesis that Cul3 regulates ion balance and blood pressure by one or more different mechanisms besides the degradation of WNK4, an idea that will be explored further in chapter three.

The Cul3 mutations that cause this disease result in the skipping of exon 9 (encoding amino acids 403-459) of the Cul3 protein. These mutations are dominant and some, including the Cul3 mutation, are believed to be de novo mutations (Boyden et al. 2012). Previous researchers have hypothesized that the Cul3 mutation is a dominant negative (Boyden et al. 2012). However, we speculated that this idea was unlikely since Cul3 has been previously shown to be essential for both embryonic development and cell survival (Singer et al. 1999; McEvoy et al. 2007). We have since demonstrated that the hypertension mutant, Cul3 $\Delta 403-459$, is a functional ubiquitin ligase capable of ubiquitinating substrates as well as the BTB protein Klhl3 (McCormick et al. 2014).

\section{CUL3 AND DISEASE: Neuronal, eye, and skin conditions}

Besides hypertension and cancer, Cul3 has also been implicated in eye, skin, and neurological conditions. Recent work has shown that Cul3 uses the substrate adaptor Klhl24 to ubiquitinate keratin14 and that dominant mutations in Klhl24 result in an increase in keratin degradation and skin fragility (Lin et al. 2016). Additionally, recent research has shown that regulation of RhoA by Cul3 and the substrate adaptor KCTD13 is necessary for brain development (Lin et al. 2015). Mutations in components of Cul3 complexes, including mutations in Cul3 itself, have 
been associated with autism, although a mechanism by which Cul3 might lead to autism has not been proposed (Codina-Sola et al. 2015; Lin et al. 2015; Wang et al. 2016). Another BTB protein, RCBTB1, has been associated with retinopathy and retinitis pigmentosa (Coppieters et al. 2016; Wu et al. 2016). Together, these findings indicate that Cul3 is essential for proper cellular and bodily functions in humans. Further study that seeks to determine the mechanisms by which Cul3 functions as well as the identity of more Cul3 substrates will be necessary in order to understand a vast array of human diseases.

\section{SIGNIFICANCE}

In this chapter, I have given a brief overview of the Cul3 ubiquitin ligase and its involvement in the degradation of cyclin $\mathrm{E}$ as well as its role in a variety of other cellular pathways and human disease. In the upcoming chapters, I will provide new insights into the role of $\mathrm{Cul} 3$ in cells including its involvement in cyclin $\mathrm{E}$ destruction. The data presented in the next chapter will demonstrate that the lysine residue on cyclin $\mathrm{E}$ that Cul3 ubiquitinates is located near the $\mathrm{N}$-terminus of cyclin $\mathrm{E}$. This detail provides insight into the role of Cul3 in cancer and tumorigenesis as cyclin E lacking its $\mathrm{N}$-terminus is associated with cancer. Chapter three will further discuss the role of Cul3 and Klhl3 in hypertension. Lastly, chapter four will describe interactions between LRR (leucine rich repeat)-domain containing proteins and Cul3 complexes as well as some other potential future directions for these projects. 


\section{REFERENCES}

Abbas T, Jha S, Sherman NE, Dutta A. 2007. Autocatalytic phosphorylation of CDK2 at the activating Thr160. Cell cycle 6: 843-852.

Akli S, Zheng PJ, Multani AS, Wingate HF, Pathak S, Zhang N, Tucker SL, Chang S, Keyomarsi K. 2004. Tumor-specific low molecular weight forms of cyclin E induce genomic instability and resistance to p21, p27, and antiestrogens in breast cancer. Cancer research 64: 3198-3208.

Ayad NG. 2005. CDKs give Cdc6 a license to drive into S phase. Cell 122: 825-827.

Bagheri-Yarmand R, Biernacka A, Hunt KK, Keyomarsi K. 2010. Low molecular weight cyclin E overexpression shortens mitosis, leading to chromosome missegregation and centrosome amplification. Cancer research 70: 50745084 .

Bernassola F, Karin M, Ciechanover A, Melino G. 2008. The HECT family of E3 ubiquitin ligases: multiple players in cancer development. Cancer Cell 14: 1021.

Besson A, Dowdy SF, Roberts JM. 2008. CDK inhibitors: cell cycle regulators and beyond. Developmental cell 14: 159-169.

Bhaskaran N, van Drogen F, Ng HF, Kumar R, Ekholm-Reed S, Peter M, Sangfelt O, Reed SI. 2013. Fbw7alpha and Fbw7gamma collaborate to shuttle cyclin E1 into the nucleolus for multiubiquitylation. Molecular and cellular biology 33: 85-97.

Blagosklonny MV, Pardee AB. 2002. The restriction point of the cell cycle. Cell cycle 1: 103-110.

Boyden LM, Choi M, Choate KA, Nelson-Williams CJ, Farhi A, Toka HR, Tikhonova IR, Bjornson R, Gharavi AG, Goilav B et al. 2012. Mutations in kelch-like 3 and cullin 3 cause hypertension and electrolyte abnormalities. Nature Genetics: 111.

Chavez-Canales M, Zhang C, Soukaseum C, Moreno E, Pacheco-Alvarez D, VidalPetiot E, Castaneda-Bueno M, Vazquez N, Rojas-Vega L, Meermeier NP et al. 2014. WNK-SPAK-NCC cascade revisited: WNK1 stimulates the activity of the $\mathrm{Na}-\mathrm{Cl}$ cotransporter via SPAK, an effect antagonized by WNK4. Hypertension ( 1979) 64: 1047-1053.

Chen HY, Chen RH. 2016. Cullin 3 Ubiquitin Ligases in Cancer Biology: Functions and Therapeutic Implications. Frontiers in oncology 6: 113.

Cheung TH, Rando TA. 2013. Molecular regulation of stem cell quiescence. Nature reviews Molecular cell biology 14: 329-340.

Choi YM, Kim KB, Lee JH, Chun YK, An IS, An S, Bae S. 2016. DBC2/RhoBTB2 functions as a tumor suppressor protein via Musashi-2 ubiquitination in breast cancer. Oncogene.

Chou PY, Fasman GD. 1975. The conformation of glucagon: predictions and consequences. Biochemistry 14: 2536-2541.

Clurman BE, Sheaff RJ, Thress K, Groudine M, Roberts JM. 1996. Turnover of cyclin E by the ubiquitin-proteasome pathway is regulated by cdk2 binding and cyclin phosphorylation. Genes Dev 10: 1979-1990. 
Codina-Sola M, Rodriguez-Santiago B, Homs A, Santoyo J, Rigau M, Aznar-Lain G, Del Campo M, Gener B, Gabau E, Botella MP et al. 2015. Integrated analysis of whole-exome sequencing and transcriptome profiling in males with autism spectrum disorders. Molecular autism 6: 21.

Cole AJ, Clifton-Bligh R, Marsh DJ. 2015. Histone H2B monoubiquitination: roles to play in human malignancy. Endocrine-related cancer 22: T19-33.

Coppieters F, Ascari G, Dannhausen K, Nikopoulos K, Peelman F, Karlstetter M, Xu M, Brachet C, Meunier I, Tsilimbaris MK et al. 2016. Isolated and Syndromic Retinal Dystrophy Caused by Biallelic Mutations in RCBTB1, a Gene Implicated in Ubiquitination. American journal of human genetics 99: 470480.

Cullinan SB, Gordan JD, Jin J, Harper JW, Diehl JA. 2004. The Keap1-BTB protein is an adaptor that bridges Nrf2 to a Cul3-based E3 ligase: oxidative stress sensing by a Cul3-Keap1 ligase. Molecular and cellular biology 24: 8477-8486.

Cummings CM, Bentley CA, Perdue SA, Baas PW, Singer JD. 2009. The Cul3/Klhdc5 E3 ligase regulates p60/katanin and is required for normal mitosis in mammalian cells. The Journal of biological chemistry 284: 11663-11675.

Delk NA, Hunt KK, Keyomarsi K. 2009. Altered subcellular localization of tumorspecific cyclin $\mathrm{E}$ isoforms affects cyclin-dependent kinase 2 complex formation and proteasomal regulation. Cancer research 69: 2817-2825.

Duda DM, Borg LA, Scott DC, Hunt HW, Hammel M, Schulman BA. 2008. Structural insights into NEDD8 activation of cullin-RING ligases: conformational control of conjugation. Cell 134: 995-1006.

Duong MT, Akli S, Wei C, Wingate HF, Liu W, Lu Y, Yi M, Mills GB, Hunt KK, Keyomarsi K. 2012. LMW-E/CDK2 deregulates acinar morphogenesis, induces tumorigenesis, and associates with the activated b-Raf-ERK1/2mTOR pathway in breast cancer patients. PLoS genetics 8: e1002538.

Ekholm SV, Reed SI. 2000. Regulation of G(1) cyclin-dependent kinases in the mammalian cell cycle. Current opinion in cell biology 12: 676-684.

Finley D, Ozkaynak E, Varshavsky A. 1987. The yeast polyubiquitin gene is essential for resistance to high temperatures, starvation, and other stresses. Cell 48: 1035-1046.

Ford C, Chevalier S. 1995. DNA replication. Almost licensed. Current biology : CB 5: 1009-1012.

Furukawa M, Xiong Y. 2005. BTB protein Keap1 targets antioxidant transcription factor Nrf2 for ubiquitination by the Cullin 3-Roc1 ligase. Molecular and cellular biology 25: 162-171.

Geng Y, Yu Q, Sicinska E, Das M, Schneider JE, Bhattacharya S, Rideout WM, Bronson RT, Gardner H, Sicinski P. 2003. Cyclin E ablation in the mouse. Cell 114: 431443.

Genschik P, Sumara I, Lechner E. 2013. The emerging family of CULLIN3-RING ubiquitin ligases (CRL3s): cellular functions and disease implications. The EMBO journal 32: 2307-2320. 
Geyer R, Wee S, Anderson S, Yates J, Wolf DA. 2003. BTB/POZ domain proteins are putative substrate adaptors for cullin 3 ubiquitin ligases. Molecular cell 12: 783-790.

Golbang AP, Cope G, Hamad A, Murthy M, Liu CH, Cuthbert AW, O'Shaughnessy K M. 2006. Regulation of the expression of the $\mathrm{Na} / \mathrm{Cl}$ cotransporter by WNK4 and WNK1: evidence that accelerated dynamin-dependent endocytosis is not involved. American journal of physiology Renal physiology 291: F1369-1376.

Gonen H, Stancovski I, Shkedy D, Hadari T, Bercovich B, Bengal E, Mesilati S, AbuHatoum O, Schwartz AL, Ciechanover A. 1996. Isolation, characterization, and partial purification of a novel ubiquitin-protein ligase, E3. Targeting of protein substrates via multiple and distinct recognition signals and conjugating enzymes. The Journal of biological chemistry 271: 302-310.

Grice GL, Nathan JA. 2016. The recognition of ubiquitinated proteins by the proteasome. Cellular and molecular life sciences : CMLS 73: 3497-3506.

Haas AL, Rose IA. 1982. The mechanism of ubiquitin activating enzyme. A kinetic and equilibrium analysis. The Journal of biological chemistry 257: 1032910337.

Haas AL, Warms JV, Hershko A, Rose IA. 1982. Ubiquitin-activating enzyme. Mechanism and role in protein-ubiquitin conjugation. The Journal of biological chemistry 257: 2543-2548.

Hao B, Oehlmann S, Sowa ME, Harper JW, Pavletich NP. 2007. Structure of a Fbw7Skp1-cyclin E complex: multisite-phosphorylated substrate recognition by SCF ubiquitin ligases. Molecular cell 26: 131-143.

Harder B, Jiang T, Wu T, Tao S, Rojo de la Vega M, Tian W, Chapman E, Zhang DD. 2015. Molecular mechanisms of Nrf2 regulation and how these influence chemical modulation for disease intervention. Biochemical Society transactions 43: 680-686.

Harwell RM, Porter DC, Danes C, Keyomarsi K. 2000. Processing of cyclin E differs between normal and tumor breast cells. Cancer research 60: 481-489.

Hicke L. 2001. Protein regulation by monoubiquitin. Nature reviews Molecular cell biology 2: 195-201.

Holmstrom KM, Kostov RV, Dinkova-Kostova AT. 2016. The multifaceted role of Nrf2 in mitochondrial function. Current opinion in toxicology 1: 80-91.

Honda R, Lowe ED, Dubinina E, Skamnaki V, Cook A, Brown NR, Johnson LN. 2005. The structure of cyclin E1/CDK2: implications for CDK2 activation and CDK2independent roles. The EMBO journal 24: 452-463.

Hoorn EJ, Ellison DH. 2012. WNK kinases and the kidney. Experimental cell research 318: 1020-1026.

Hori T, Osaka F, Chiba T, Miyamoto C, Okabayashi K, Shimbara N, Kato S, Tanaka K. 1999. Covalent modification of all members of human cullin family proteins by NEDD8. Oncogene 18: 6829-6834.

Ibeawuchi SR, Agbor LN, Quelle FW, Sigmund CD. 2015. Hypertension-causing Mutations in Cullin3 Protein Impair RhoA Protein Ubiquitination and Augment the Association with Substrate Adaptors. The Journal of biological chemistry 290: 19208-19217. 
Itoh K, Wakabayashi N, Katoh Y, Ishii T, Igarashi K, Engel JD, Yamamoto M. 1999. Keap1 represses nuclear activation of antioxidant responsive elements by Nrf2 through binding to the amino-terminal Neh2 domain. Genes Dev 13: 7686.

Jiang J. 2006. Regulation of Hh/Gli signaling by dual ubiquitin pathways. Cell cycle $\mathbf{5}$ : 2457-2463.

Jin J, Harper JW. 2002. RING finger specificity in SCF-driven protein destruction. Developmental cell 2: 685-687.

Johnson JL, Lu C, Raharjo E, McNally K, McNally FJ, Mains PE. 2009. Levels of the ubiquitin ligase substrate adaptor MEL-26 are inversely correlated with MEI$1 /$ katanin microtubule-severing activity during both meiosis and mitosis. Developmental biology 330: 349-357.

Kawakami T, Chiba T, Suzuki T, Iwai K, Yamanaka K, Minato N, Suzuki H, Shimbara $\mathrm{N}$, Hidaka Y, Osaka F et al. 2001. NEDD8 recruits E2-ubiquitin to SCF E3 ligase. The EMBO journal 20: 4003-4012.

Keck JM, Summers MK, Tedesco D, Ekholm-Reed S, Chuang LC, Jackson PK, Reed SI. 2007. Cyclin $\mathrm{E}$ overexpression impairs progression through mitosis by inhibiting APC(Cdh1). The Journal of cell biology 178: 371-385.

Kelly BL, Wolfe KG, Roberts JM. 1998. Identification of a substrate-targeting domain in cyclin E necessary for phosphorylation of the retinoblastoma protein. Proceedings of the National Academy of Sciences of the United States of America 95: 2535-2540.

Kim J, Keum YS. 2016. NRF2, a Key Regulator of Antioxidants with Two Faces towards Cancer. Oxidative medicine and cellular longevity 2016: 2746457.

Kleiger G, Hao B, Mohl DA, Deshaies RJ. 2009. The acidic tail of the Cdc34 ubiquitinconjugating enzyme functions in both binding to and catalysis with ubiquitin ligase SCFCdc4. The Journal of biological chemistry 284: 36012-36023.

Kobayashi A, Kang MI, Okawa H, Ohtsuji M, Zenke Y, Chiba T, Igarashi K, Yamamoto M. 2004. Oxidative stress sensor Keap1 functions as an adaptor for Cul3based E3 ligase to regulate proteasomal degradation of Nrf2. Molecular and cellular biology 24: 7130-7139.

Koepp DM, Schaefer LK, Ye X, Keyomarsi K, Chu C, Harper JW, Elledge SJ. 2001. Phosphorylation-dependent ubiquitination of cyclin E by the SCFFbw7 ubiquitin ligase. Science 294: 173-177.

Koff A, Cross F, Fisher A, Schumacher J, Leguellec K, Philippe M, Roberts JM. 1991. Human cyclin E, a new cyclin that interacts with two members of the CDC2 gene family. Cell 66: 1217-1228.

Koff A, Giordano A, Desai D, Yamashita K, Harper JW, Elledge S, Nishimoto T, Morgan DO, Franza BR, Roberts JM. 1992. Formation and activation of a cyclin E-cdk2 complex during the G1 phase of the human cell cycle. Science (New York, NY) 257: $1689-1694$.

Kwon JE, La M, Oh KH, Oh YM, Kim GR, Seol JH, Baek SH, Chiba T, Tanaka K, Bang OS et al. 2006. BTB domain-containing speckle-type POZ protein (SPOP) serves as an adaptor of Daxx for ubiquitination by Cul3-based ubiquitin ligase. The Journal of biological chemistry 281: 12664-12672. 
Leinonen HM, Kansanen E, Polonen P, Heinaniemi M, Levonen AL. 2015. Dysregulation of the Keap1-Nrf2 pathway in cancer. Biochemical Society transactions 43: 645-649.

Libertini SJ, Robinson BS, Dhillon NK, Glick D, George M, Dandekar S, Gregg JP, Sawai E, Mudryj M. 2005. Cyclin E both regulates and is regulated by calpain 2, a protease associated with metastatic breast cancer phenotype. Cancer research 65: 10700-10708.

Lim KL, Lim GG. 2011. K63-linked ubiquitination and neurodegeneration. Neurobiology of disease 43: 9-16.

Lin GN, Corominas R, Lemmens I, Yang X, Tavernier J, Hill DE, Vidal M, Sebat J, Iakoucheva LM. 2015. Spatiotemporal 16p11.2 protein network implicates cortical late mid-fetal brain development and KCTD13-Cul3-RhoA pathway in psychiatric diseases. Neuron 85: 742-754.

Lin Z, Li S, Feng C, Yang S, Wang H, Ma D, Zhang J, Gou M, Bu D, Zhang T et al. 2016. Stabilizing mutations of KLHL24 ubiquitin ligase cause loss of keratin 14 and human skin fragility. Nat Genet.

Loeb KR, Kostner H, Firpo E, Norwood T, Tsuchiya KD, Clurman BE, Roberts JM. 2005. A mouse model for cyclin E-dependent genetic instability and tumorigenesis. Cancer Cell 8: 35-47.

Louis-Dit-Picard H, Barc J, Trujillano D, Miserey-Lenkei S, Bouatia-Naji N, Pylypenko O, Schott J-J, Jeunemaitre X. 2012. KLHL3 mutations cause familial hyperkalemic hypertension by impairing ion transport in the distal nephron. Nature Genetics 44: 456-460.

Lu A, Pfeffer SR. 2013. Golgi-associated RhoBTB3 targets cyclin E for ubiquitylation and promotes cell cycle progression. The Journal of cell biology 203: 233-250.

Mailand N, Diffley JF. 2005. CDKs promote DNA replication origin licensing in human cells by protecting Cdc6 from APC/C-dependent proteolysis. Cell 122: 915-926.

Mani RS. 2014. The emerging role of speckle-type POZ protein (SPOP) in cancer development. Drug discovery today 19: 1498-1502.

McCormick JA, Yang CL, Zhang C, Davidge B, Blankenstein KI, Terker AS, Yarbrough B, Meermeier NP, Park HJ, McCully B et al. 2014. Hyperkalemic hypertensionassociated cullin 3 promotes WNK signaling by degrading KLHL3. The Journal of clinical investigation 124: 4723-4736.

McEvoy J, Kossatz U, Malek N, Singer J. 2007. Constitutive turnover of cylin E by Cul3 maintains quiescence. Molecular Cell Biology 27: 3651-3666.

Metzger MB, Hristova VA, Weissman AM. 2012. HECT and RING finger families of E3 ubiquitin ligases at a glance. Journal of cell science 125: 531-537.

Metzger MB, Pruneda JN, Klevit RE, Weissman AM. 2014. RING-type E3 ligases: master manipulators of E2 ubiquitin-conjugating enzymes and ubiquitination. Biochimica et biophysica acta 1843: 47-60.

Minella AC, Loeb KR, Knecht A, Welcker M, Varnum-Finney BJ, Bernstein ID, Roberts JM, Clurman BE. 2008. Cyclin E phosphorylation regulates cell proliferation in hematopoietic and epithelial lineages in vivo. Genes Dev 22: 1677-1689. 
Nishitani H, Lygerou Z. 2002. Control of DNA replication licensing in a cell cycle. Genes to cells : devoted to molecular \& cellular mechanisms 7: 523-534.

O'Shaughnessy KM. 2015. Gordon Syndrome: a continuing story. Pediatric nephrology (Berlin, Germany) 30: 1903-1908.

Ohta A, Schumacher F-R, Mehellou Y, Johnson C, Knebel A, Macartney TJ, Wood NT, Alessi DR, Kurz T. 2013. The CUL3-KLHL3 E3 ligse complex mutated in Gordon's hypertension syndrome interacts with and ubiquitylates WNK isoforms: disease causing mutations in Klhle and WNK4 disrupt interaction. Biochemical Journal 451: 111-122.

Ohtsubo M, Roberts JM. 1993. Cyclin-dependent regulation of G1 in mammalian fibroblasts. Science 259: 1908-1912.

Pardee AB. 1974. A restriction point for control of normal animal cell proliferation. Proceedings of the National Academy of Sciences of the United States of America 71: 1286-1290.

Penkert J, Ripperger T, Schieck M, Schlegelberger B, Steinemann D, Illig T. 2016. On metabolic reprogramming and tumor biology: A comprehensive survey of metabolism in breast cancer. Oncotarget 7: 67626-67649.

Perez-Neut M, Shum A, Cuevas BD, Miller R, Gentile S. 2015. Stimulation of hERG1 channel activity promotes a calcium-dependent degradation of cyclin E2, but not cyclin E1, in breast cancer cells. Oncotarget 6: 1631-1639.

Petroski MD, Deshaies RJ. 2005. Function and regulation of cullin-RING ubiquitin ligases. Nature reviews Molecular cell biology 6: 9-20.

Pickart CM, Rose IA. 1985. Functional heterogeneity of ubiquitin carrier proteins. The Journal of biological chemistry 260: 1573-1581.

Pintard L, Willems A, Peter M. 2004. Cullin-based ubiquitin ligases: Cul3-BTB complexes join the family. The EMBO journal 23: 1681-1687.

Pintard L, Willis JH, Willems A, Johnson JL, Srayko M, Kurz T, Glaser S, Mains PE, Tyers M, Bowerman B et al. 2003. The BTB protein MEL-26 is a substratespecific adaptor of the CUL-3 ubiquitin-ligase. Nature 425: 311-316.

Plafker KS, Singer JD, Plafker SM. 2009. The ubiquitin conjugating enzyme, UbcM2, engages in novel interactions with components of cullin-3 based E3 ligases. Biochemistry 48: 3527-3537.

Porter DC, Keyomarsi K. 2000. Novel splice variants of cyclin E with altered substrate specificity. Nucleic acids research 28: E101.

Porter DC, Zhang N, Danes C, McGahren MJ, Harwell RM, Faruki S, Keyomarsi K. 2001. Tumor-specific proteolytic processing of cyclin $\mathrm{E}$ generates hyperactive lower-molecular-weight forms. Molecular and cellular biology 21: 6254-6269.

Rath SL, Senapati S. 2014. Why are the truncated cyclin Es more effective CDK2 activators than the full-length isoforms? Biochemistry 53: 4612-4624.

Resnitzky D, Gossen M, Bujard H, Reed SI. 1994. Acceleration of the G1/S phase transition by expression of cyclins D1 and E with an inducible system. Molecular and cellular biology 14: 1669-1679. 
Richardson HE, O'Keefe LV, Reed SI, Saint R. 1993. A Drosophila G1-specific cyclin E homolog exhibits different modes of expression during embryogenesis. Development 119: 673-690.

Rogers S, Wells R, Rechsteiner M. 1986. Amino acid sequences common to rapidly degraded proteins: the PEST hypothesis. Science 234: 364-368.

Rogers SW, Rechsteiner MC. 1986. Microinjection studies on selective protein degradation: relationships between stability, structure, and location. Biomedica biochimica acta 45: 1611-1618.

Said TK, Medina D. 1995. Cell cyclins and cyclin-dependent kinase activities in mouse mammary tumor development. Carcinogenesis 16: 823-830.

Scuderi R, Palucka KA, Pokrovskaja K, Bjorkholm M, Wiman KG, Pisa P. 1996. Cyclin $\mathrm{E}$ overexpression in relapsed adult acute lymphoblastic leukemias of B-cell lineage. Blood 87: 3360-3367.

Sherr CJ. 1994. G1 phase progression: cycling on cue. Cell 79: 551-555.

Sherr CJ, Roberts JM. 1999. CDK inhibitors: positive and negative regulators of G1phase progression. Genes Dev 13: 1501-1512.

Shibata S, Zhang J, Puthumana J, Stone KL, Lifton RP. 2013. Kelch-like 3 and Cullin 3 regulate electrolyte homeostasis via ubiquitination and deradation of WNK4. PNAS 110: 7838-7843.

Siddiqi S, Sussman MA. 2014. The heart: mostly postmitotic or mostly premitotic? Myocyte cell cycle, senescence, and quiescence. The Canadian journal of cardiology 30: 1270-1278.

Singer JD, Gurian-West M, Clurman B, Roberts JM. 1999. Cullin-3 targets cyclin E for ubiquitination and controls S phase in mammalian cells. Genes Dev 13: 23752387.

Skowyra D, Craig KL, Tyers M, Elledge SJ, Harper JW. 1997. F-box proteins are receptors that recruit phosphorylated substrates to the SCF ubiquitin-ligase complex. Cell 91: 209-219.

Sparaneo A, Fabrizio FP, Muscarella LA. 2016. Nrf2 and Notch Signaling in Lung Cancer: Near the Crossroad. Oxidative medicine and cellular longevity 2016: 7316492.

Spruck CH, Won KA, Reed SI. 1999. Deregulated cyclin E induces chromosome instability. Nature 401: 297-300.

Stogios PJ, Downs GS, Jauhal JJ, Nandra SK, Prive GG. 2005. Sequence and structural analysis of BTB domain proteins. Genome biology 6: R82.

Strohmaier H, Spruck CH, Kaiser P, Won KA, Sangfelt O, Reed SI. 2001. Human F-box protein hCdc4 targets cyclin $\mathrm{E}$ for proteolysis and is mutated in a breast cancer cell line. Nature 413: 316-322.

Swiss VA, Casaccia P. 2010. Cell-context specific role of the E2F/Rb pathway in development and disease. Glia 58: 377-390.

Tyers M, Jorgensen P. 2000. Proteolysis and the cell cycle: with this RING I do thee destroy. Current opinion in genetics \& development 10: 54-64.

Wakabayashi M, Mori T, Isobe K, Sohara E, Susa K, Araki Y, Chiga M, Kikuchi E, Nomura N, Mori $Y$ et al. 2013. Impaired KLHL3-mediated Ubiquitination of WNK4 Causes Human Hypertenison. Cell Reports 2013: 1-11. 
Wang T, Guo H, Xiong B, Stessman HA, Wu H, Coe BP, Turner TN, Liu Y, Zhao W, Hoekzema K et al. 2016. De novo genic mutations among a Chinese autism spectrum disorder cohort. Nature communications 7: 13316.

Wang XD, Rosales JL, Magliocco A, Gnanakumar R, Lee KY. 2003. Cyclin E in breast tumors is cleaved into its low molecular weight forms by calpain. Oncogene 22: 769-774.

Welcker M, Singer J, Loeb KR, Grim J, Bloecher A, Gurien-West M, Clurman BE, Roberts JM. 2003. Multisite phosphorylation by Cdk2 and GSK3 controls cyclin E degradation. Molecular cell 12: 381-392.

Wimuttisuk W, Singer JD. 2007. The Cullin3 ubiquitin ligase functions as a Nedd8bound heterodimer. Molecular biology of the cell 18: 899-909.

Wimuttisuk W, West M, Davidge B, Yu K, Salomon A, Singer JD. 2014. Novel Cul3 binding proteins function to remodel E3 ligase complexes. BMC cell biology 15: 28.

Wingate H, Zhang N, McGarhen MJ, Bedrosian I, Harper JW, Keyomarsi K. 2005. The tumor-specific hyperactive forms of cyclin $\mathrm{E}$ are resistant to inhibition by p21 and p27. The Journal of biological chemistry 280: 15148-15157.

Won KA, Reed SI. 1996. Activation of cyclin E/CDK2 is coupled to site-specific autophosphorylation and ubiquitin-dependent degradation of cyclin $\mathrm{E}$. The EMBO journal 15: 4182-4193.

Wu JH, Liu JH, Ko YC, Wang CT, Chung YC, Chu KC, Liu TT, Chao HM, Jiang YJ, Chen SJ et al. 2016. Haploinsufficiency of RCBTB1 is associated with Coats disease and familial exudative vitreoretinopathy. Human molecular genetics $\mathbf{2 5}$ 1637-1647.

Xu L, Wei Y, Reboul J, Vaglio P, Shin TH, Vidal M, Elledge SJ, Harper JW. 2003. BTB proteins are substrate-specific adaptors in an SCF-like modular ubiquitin ligase containing CUL-3. Nature 425: 316-321.

Yang CL, Angell J, Mitchell R, Ellison DH. 2003. WNK kinases regulate thiazidesensitive Na-Cl cotransport. The Journal of clinical investigation 111: 10391045.

Zhang CS, Liu Q, Li M, Lin SY, Peng Y, Wu D, Li TY, Fu Q, Jia W, Wang X et al. 2015. RHOBTB3 promotes proteasomal degradation of HIFalpha through facilitating hydroxylation and suppresses the Warburg effect. Cell research 25: $1025-1042$.

Zhang DD, Lo SC, Cross JV, Templeton DJ, Hannink M. 2004. Keap1 is a redoxregulated substrate adaptor protein for a Cul3-dependent ubiquitin ligase complex. Molecular and cellular biology 24: 10941-10953.

Zhang P, Gao K, Tang Y, Jin X, An J, Yu H, Wang H, Zhang Y, Wang D, Huang H et al. 2014. Destruction of DDIT3/CHOP protein by wild-type SPOP but not prostate cancer-associated mutants. Human mutation.

Zheng N, Schulman BA, Song L, Miller JJ, Jeffrey PD, Wang P, Chu C, Koepp DM, Elledge SJ, Pagano M et al. 2002. Structure of the Cul1-Rbx1-Skp1-F boxSkp2 SCF ubiquitin ligase complex. Nature 416: 703-709. 
CHAPTER 2: The N-terminus of Cyclin E is Required for Cul3-mediated Degradation 


\section{INTRODUCTION AND BACKGROUND:}

Cyclin $\mathrm{E}$ and its binding partner $\mathrm{Cdk} 2$ regulate the transition from $\mathrm{G}_{1}$ to $\mathrm{S}$ phase as well as release from quiescence in mammalian cells (Koff et al. 1991; Geng et al. 2003). Thus, it is not surprising that cell cycle errors are associated with alterations to cyclin E function and/or abundance; fibroblasts lacking both cyclin E genes, cyclin E1 and cyclin E2, are unable to release from quiescence (Geng et al. 2003). In contrast, overexpression of cyclin $E$ is associated with cancer and tumorigenesis (Said and Medina 1995). Analysis of the cyclin E protein has revealed several functional domains including a central cyclin homology domain which interacts with Cdk2, a unique N-terminal region, and a C-terminal PEST sequence, which is commonly found in proteins that get degraded by the ubiquitin system (Rogers et al. 1986; Rogers and Rechsteiner 1986; Richardson et al. 1993; Honda et al. 2005; Rath and Senapati 2014). In certain cancers, including breast, ovarian and melanoma, cyclin E is known to be cleaved by proteases resulting in $\mathrm{N}$-terminally truncated low molecular weight (LMW) forms ranging in size from 33 to 45 kilodaltons (Scuderi et al. 1996; Harwell et al. 2000; Porter and Keyomarsi 2000; Porter et al. 2001; Wang et al. 2003; Libertini et al. 2005). LMW cyclin E activates Cdk2 and demonstrates increased cyclin E/Cdk2 activity (Porter et al. 2001). These forms of cyclin E are associated with poor clinical prognosis in cancer patients (Harwell et al. 2000; Porter et al. 2001; Duong et al. 2012).

Cyclin $\mathrm{E}$ expression is restricted to the $\mathrm{G}_{1} / \mathrm{S}$ transition by two distinct $\mathrm{E} 3$ ubiquitin ligase complexes which are responsible for the degradation of cyclin E: Cul1 and Cul3 (Clurman et al. 1996; Singer et al. 1999; Strohmaier et al. 2001; 
Welcker et al. 2003). Both Cul1 and Cul3 are members of the cullin-RING family of ubiquitin ligases. Cul1 or SCF (Skp1, Cul1, Fbxw7) based ligases use Fbxw7 as a substrate adaptor to recognize cyclin E (Koepp et al. 2001; Strohmaier et al. 2001; Hao et al. 2007). Cul1 mediated-degradation requires phosphorylation of cyclin $\mathrm{E}$ at T77 and T395 in order for ubiquitination of cyclin E to occur (Clurman et al. 1996; Welcker et al. 2003; Loeb et al. 2005; Minella et al. 2008). Cul1-mediated degradation of cyclin E occurs about four hours following release from a thymidine block (S-phase) (Bhaskaran et al. 2013).

In contrast to the Cul1 pathway, the mechanistic details of Cul3 mediated destruction of cyclin E remain largely uncharacterized. Similar to Cul1 complexes, Cul3 ubiquitin ligase complexes consist of a substrate adaptor (BTB protein) which binds near the Cul3 $\mathrm{N}$-terminus to recruit substrates, and C-terminal region that binds to the RING finger protein Rbx1 (Tyers and Jorgensen 2000; Jin and Harper 2002; Duda et al. 2008), which in return recruits an E2 ubiquitin conjugating enzyme (Petroski and Deshaies 2005). Previous studies from our lab have shown that Cul3 degrades cyclin E that is not bound to Cdk2 and regulation of cyclin E by Cul3 is necessary for the maintenance of quiescence in liver (Singer et al. 1999; McEvoy et al. 2007). In addition, it has recently been demonstrated that Cul3 can utilize the substrate adaptor RhoBTB3 to ubiquitinate cyclin E (Lu and Pfeffer 2013).

Despite the advances, many details regarding the mechanism utilized by Cul3 for cyclin E ubiquitination remain as yet unknown, including the location of the degron, or region recognized by the ubiquitin ligase, that Cul3 uses to recognize 
cyclin E. Here, a Cul3 degron near the N-terminus of cyclin E is identified, and a unique mechanism for Cul3-mediated cyclin E destruction is proposed.

\section{RESULTS:}

\section{Cyclin E binds directly to Cul3 independently of BTB domain-containing proteins}

Cul3 is believed to require a BTB protein to bind cyclin E, but previous work from our lab has shown Cul3 can bind cyclin E in a yeast two-hybrid screen, which suggests that the proteins interact directly with each other (Singer et al. 1999). In order to determine the nature of the interaction between Cul3 and cyclin E, and whether the two proteins interact directly with each other, several Flag-tagged Cul3 mutants were co-transfected with full-length Myc-tagged cyclin E1 and their binding was measured using immunoprecipitation. The mutants represented disruptions of the major functional regions of Cul3; the BTB domain interaction region, the Nedd8 modification site, and the gain of function 403-459 deletion (Zheng et al. 2002; Wimuttisuk and Singer 2007; Boyden et al. 2012; McCormick et al. 2014; Wimuttisuk et al. 2014). Cyclin E bound to all Cul3 mutants tested, including the Cul3 $\Delta 51-67$ mutant, which cannot bind BTB proteins (Figure 2.1A lane 3). These data imply that cyclin E is able to bind directly to Cul3 without the aid of a BTB protein, unlike what has been shown for other Cul3 substrates. 


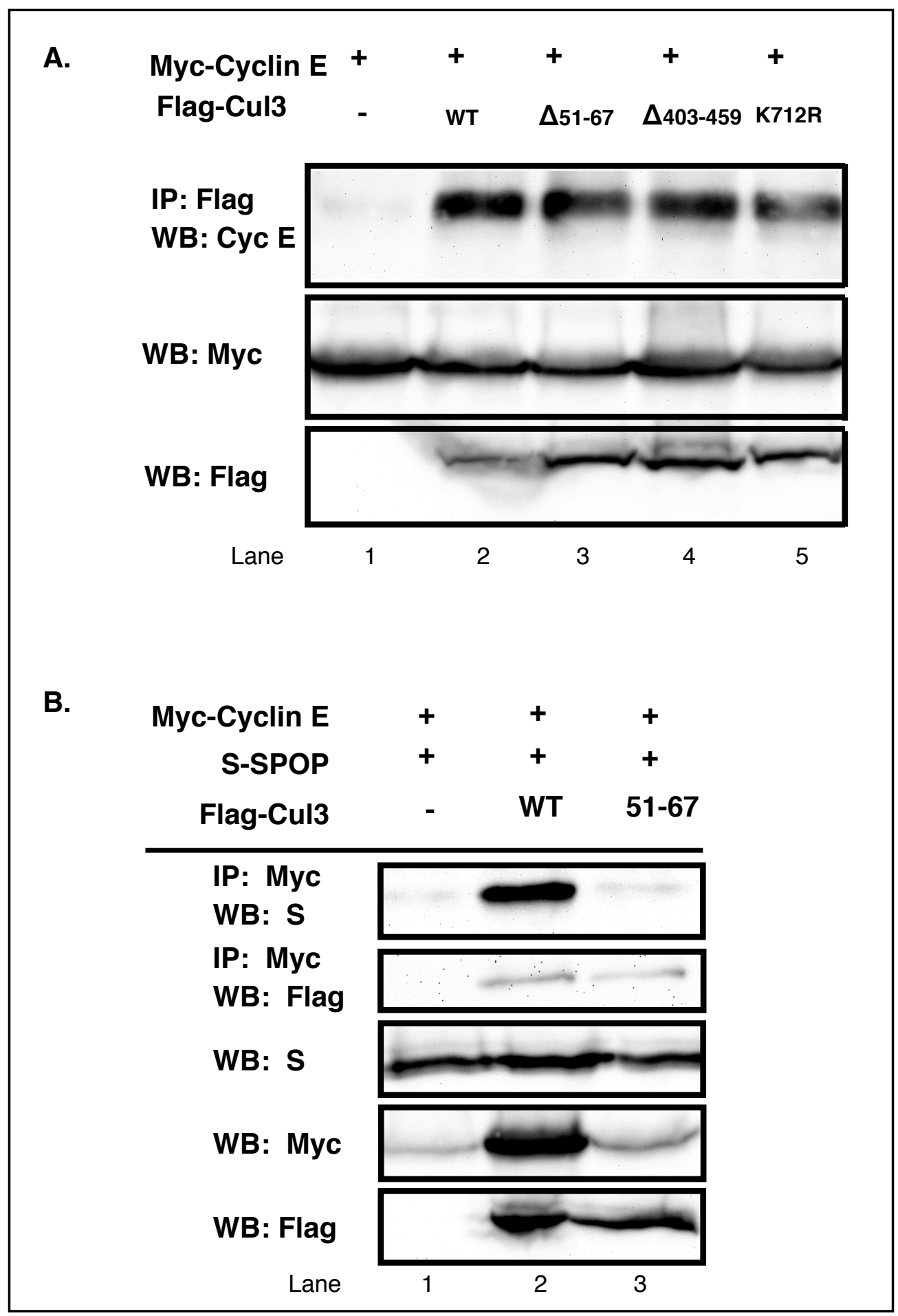

Figure 2.1: Cul3 binds cyclin E directly. A: Myc-cyclin E was co-transfected in 293 cells with different Flag-tagged Cul3 mutants including Cul3 $\Delta 51-67$, which cannot bind BTB proteins, Cul3 $\Delta 403-459$, which causes FHHt, and Cul3K712 R, which is inactive because it cannot be neddylated. Immunoprecipitations (IPs) were performed using a Flag antibody. A western blot using Myc antibody was performed to detect cyclin E. IP results are shown in the top panel. B: Flag-Cul3 or Flag-Cul3 
451-67 was co-transfected with Myc-cyclin E, HA-ubiquitin, and S-tagged-SPOP. An IP for Myc-cyclin E was performed followed by western blotting for to detect S-SPOP (B, top panel) and Flag-Cul3 (B, second panel). The lower three panels show the original protein levels of the lysates prior to immunoprecipitation.

In order to further delineate the interaction between $\mathrm{Cul} 3$ and cyclin $\mathrm{E}, \mathrm{Cul} 3$ and cyclin E1 binding was examined in the presence of SPOP, a BTB protein that does not bind or participate in cyclin E degradation (Kwon et al. 2006; Zhang et al. 2014). We found that when cyclin E1 and SPOP are co-transfected with WT Cul3, cyclin E can co-immunoprecipitate SPOP, but cyclin E was not able to coimmunoprecitpitate SPOP when the Cul3 mutant Cul3 $\Delta 51-67$, which cannot bind BTB domain-containing proteins, was used (Figure 2.1B lane 2 compared to lane 3). This shows us that Cul3 was 'linking' cyclin E to SPOP in this binding assay. The ability of cyclin E to immunopreciptitate SPOP only when it is bound to Cul3 is consistent with a direct interaction between cyclin E1 and Cul3 and that the binding interaction occurs outside of the region associated with binding BTB domaincontaining proteins. 
A.

1

Cleavage 395
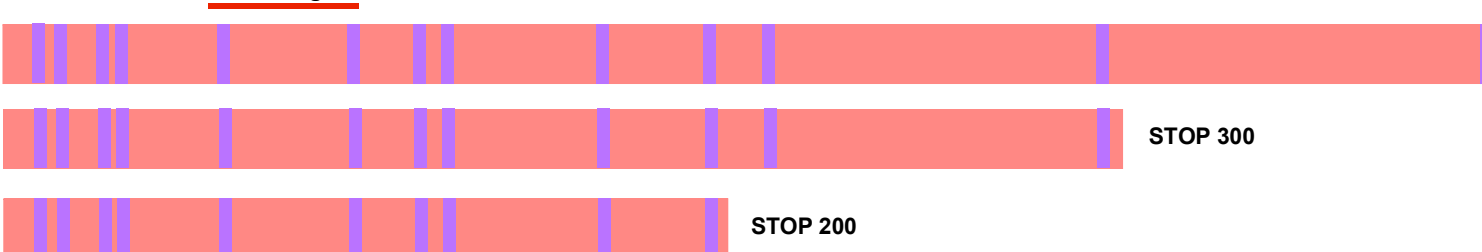

STOP 100 (Unstable)

B.

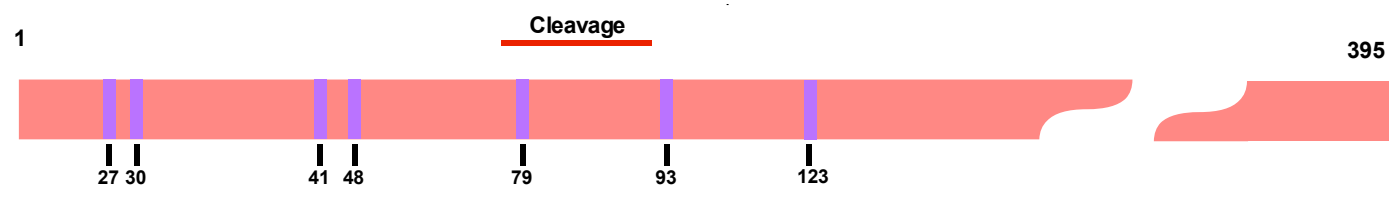

Figure 2.2: Mutants of cyclin E1. A: Cartoon showing full length, wild-type cyclin E (top) and three truncation mutants throughout the protein, STOP100, STOP200, and STOP300. B: Cartoon depicting the locations of point mutants and alanine scanning mutants (designated by amino acid number) on cyclin E. The cleavage site which produces LMW cyclin $\mathrm{E}$ is shown in red. 


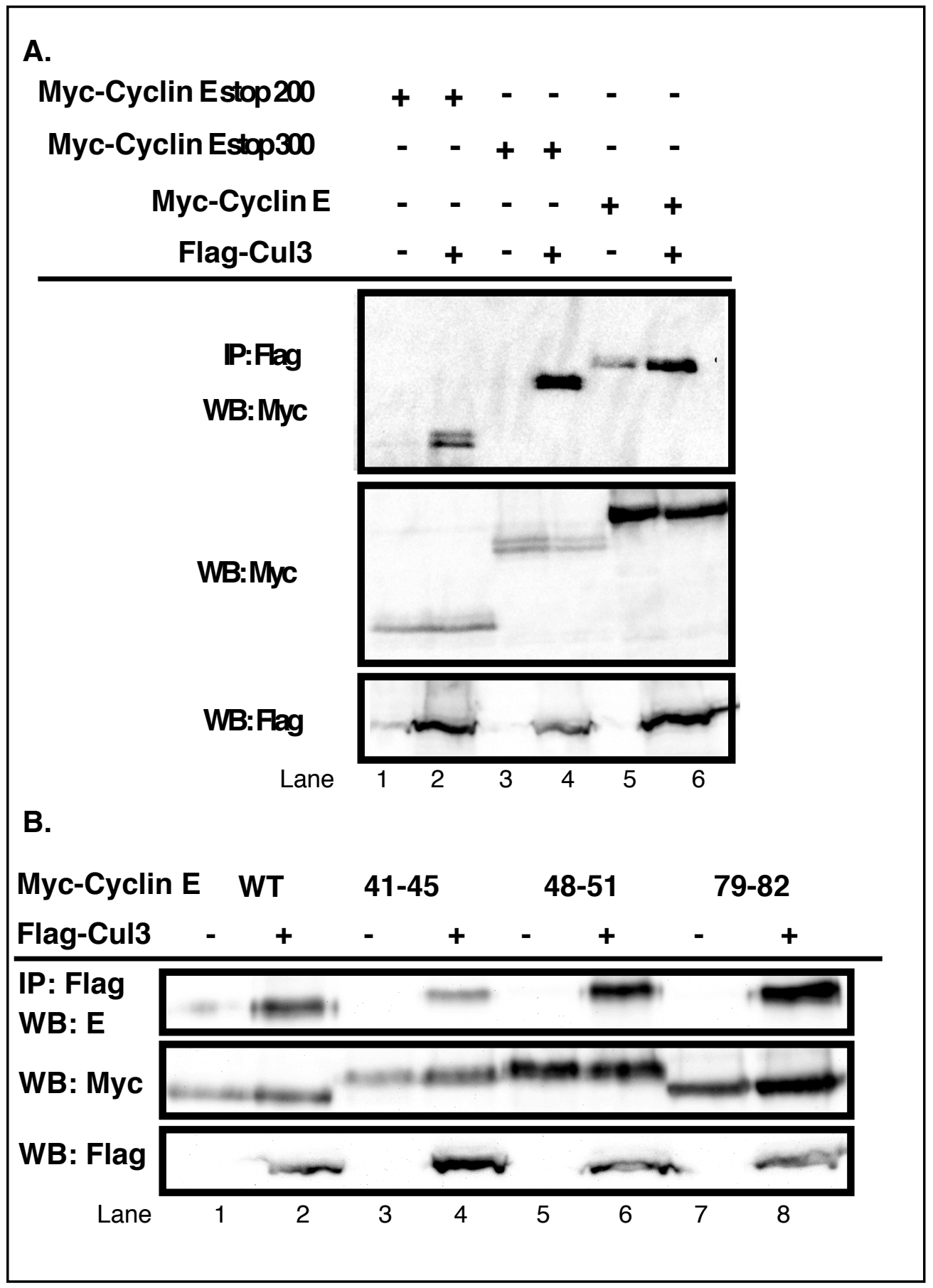

Figure 2.3: The N-terminus of cyclin $E$ interacts with Cul3. A: Upper blot, immunoprecipitation (IP) for Flag-Cul3 and blot for Myc-cyclin E showing binding. Middle and lower blots show relative levels of transfected protein in cell extracts. B: Top blot, IP for Flag-Cul3 and western blot for Myc-cyclin E showing binding of three N-terminally located alanine scanning mutants (lanes 4, 6, and 8) in comparison to wild-type cyclin E (lane 2). The lower two blots show levels of 
transfected protein in the cell extracts. All binding assays were repeated a minimum of two times. The result shown in panel B was repeated four times and is reproducible.

\section{Mutations in the N-terminal region of cyclin E prevent degradation by the Cul3 complex}

In order to determine what region of cyclin $\mathrm{E} 1$ binds to Cul3, two Cterminally truncated cyclin E1 mutants were analyzed for binding to Cul3 and compared to full-length cyclin E1. These consisted of the N-terminal 200 amino acids (STOP 200) of cyclin E and the N-terminal 300 amino acids (STOP 300) of cyclin E (Figure 2.2). A STOP100 truncation of cyclin E1 was also transfected, but it was found to be unstable and therefore not used for further experimentation (Figure 2.2). Cul3 bound to both cyclin E1 truncation mutants as well as wild type cyclin E1 (Figure 2.3A compare lanes 2 and 4 to lane 6). To further pinpoint the binding site, we examined the potential of several cyclin E1 alanine-scanning mutants for binding to Cul3 (Kelly et al. 1998). Each mutant in this set contains a charged amino acid sequence that has been mutated to alanines. Amongst the set of mutants, one alanine-scanning mutant DPDEE $\rightarrow$ AAAA (amino acids 41-45), which is located near the N-terminus, showed decreased binding to Cul3 (Figure 2.3B first panel lane 6 in comparison to lanes 2, 4, 8, and 10).

After determining that Cul3 only requires the $\mathrm{N}$-terminal portion of cyclin E1 for binding, we sought to determine if this region contains all the necessary signals for Cul3-mediated degradation to occur. In order to examine this, the stability of the different mutants was checked in a novel transfection assay utilizing cells that are deficient for Cul3. It was observed that in Cul3 hypomorphic (floxed) MEFs (Mouse 
Embryonic Fibroblasts), transfected cyclin E was more abundant than in WT MEFs, similar to the endogenous levels of cyclin E (McEvoy et al. 2007). Controls that were not Cul3 substrates transfected with equal efficiency demonstrating the specificity for Cul3 substrates in this assay. The same regulation of cyclin E was observed when comparing WT (Cul3 containing) and Cul3 KO HEK293 cells (Ibeawuchi et al. 2015) (Figure 2.4A lanes 1 and 2). We reasoned that if a mutant cyclin E, that lacked the Cul3 degron, was transfected into these two genotypes, we would not see a difference in levels of cyclin E. Therefore, transfected cyclin E1 mutants that are Cul3 substrates would be expected to express at higher levels in the Cul3 KO cells in comparison to wild-type 293 cells. On the other hand, a mutant that is not degraded in a Cul3-dependent fashion would be expected to show equal levels of transfected protein in the two cell types.

Before experimentation began, two controls Myc-cyclin E1 and lysineless cyclin E1 were transfected into the two cell types (WT and Cul3 KO 293s). We observed that when transfected with equal amounts of WT cyclin E1, the protein is detected at higher levels in the KO 293 cells than the WT cells (Figure 2.4A compare lanes 1 and 2). In contrast, lysineless cyclin E1 was expressed evenly in both cell types (Figure 2.4A lanes 3 and 4), indicating the utility of this assay as a measure of the substrate being recognized for degradation instead of merely binding. Next, the stability of the cyclin E1 truncations were examined using this assay and we observed that both the STOP 200 and STOP 300 cyclin E truncations are both more stable in the KO cells, implying that the degron recognized by Cul3 is found within the first half of the cyclin E1 protein (Figure 2.4B compare lane 1 to lane 2 and 38 
compare lane 3 to lane 4). Taken together, these results demonstrate that the Cul3 degron resides in the $\mathrm{N}$-terminal half of the cyclin, in contrast to Cul1 the degron which encompasses, in part, T395 located near the C-terminus (Clurman et al. 1996).

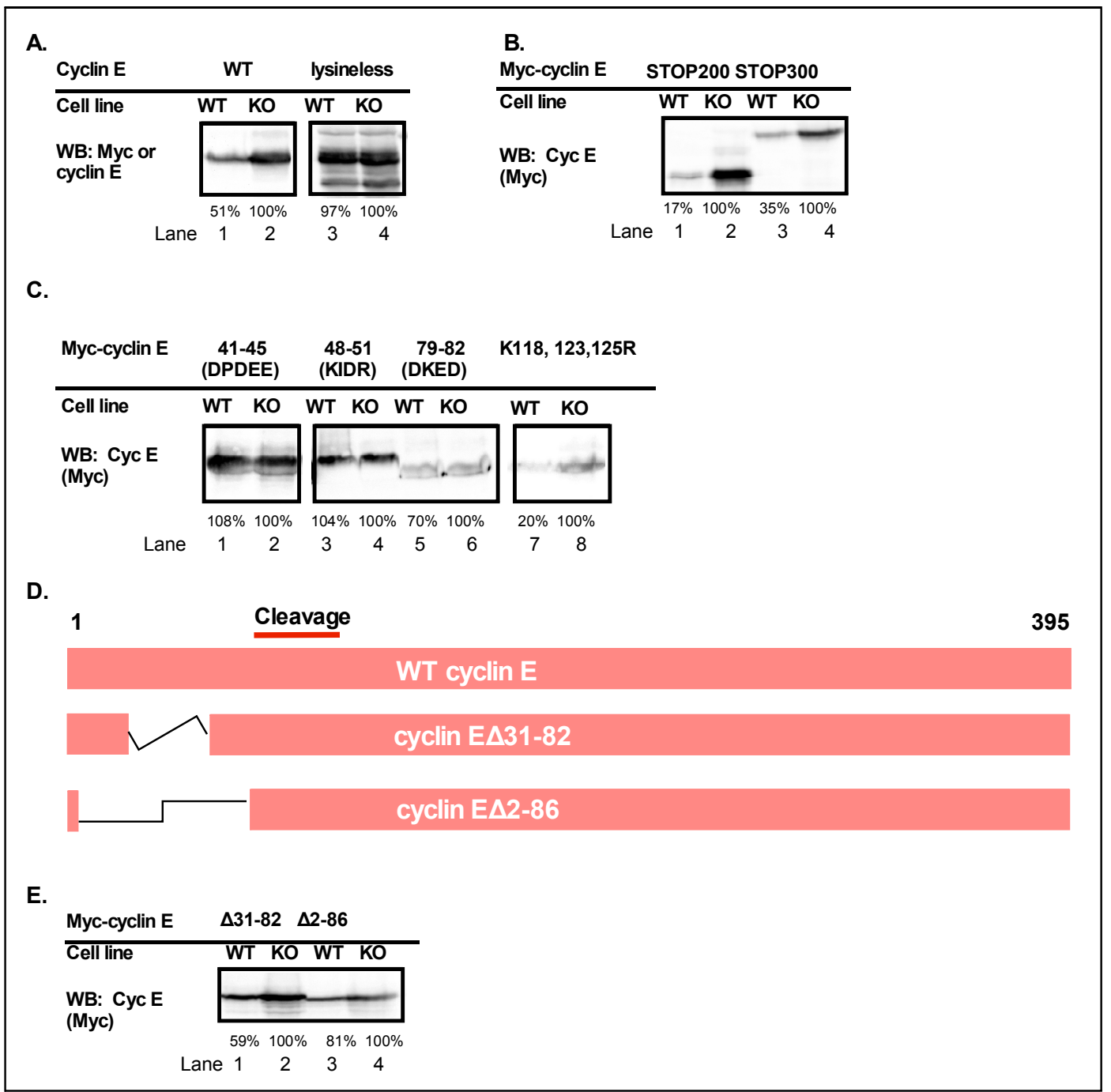

Figure 2.4: Mutations near the $N$-terminus of cyclin E result in increased stability. A: Controls for a stability assay using WT and Cul3 KO 293 cells shows that cyclin E, a Cul3 substrate, is more stable when transfected into Cul3 KO cells (A: Left panel). Lysineless cyclin E, which cannot be ubiquitinated, is shown as a negative control 
(A: Right panel). B: Myc-tagged STOP 200 and STOP 300 truncations of cyclin E were transfected into wild-type and Cul3 KO 293 cells and their expression was measured using a Myc (cyclin E) antibody. C: Three Myc-tagged alanine scanning mutants, DPDEE $\rightarrow$ AAAAA (residues 41-45, lanes 1 and 2), KIDR $\rightarrow$ AAAA (amino acids 48-51, lanes 3 and 4) and DKED $\rightarrow$ AAAA (amino acids 79-82, lanes 5 and 6), in addition to a triple point mutant, cyclin E K118R, K123R, K125R (Panel C lanes 7 and 8), were transfected into both WT and Cul3 KO cells and their abundance was measured using a Myc (cyclin E) antibody. D: Diagram showing two mutants in which portions of the N-terminal domain have been deleted. E: Expression of transfected Myc-cyclin E $\Delta 31-82$ and Myc-cyclin E $\Delta 2-86$ are shown in both the WT and KO cells (E, right panel, lanes 1 through 4). Quantification of each western blot (WB) is listed below each lane as a percent relative to the sample in the KO lane for each pair (KO is always 100 percent). Transfections were repeated a minimum of three times and representative results are shown. 


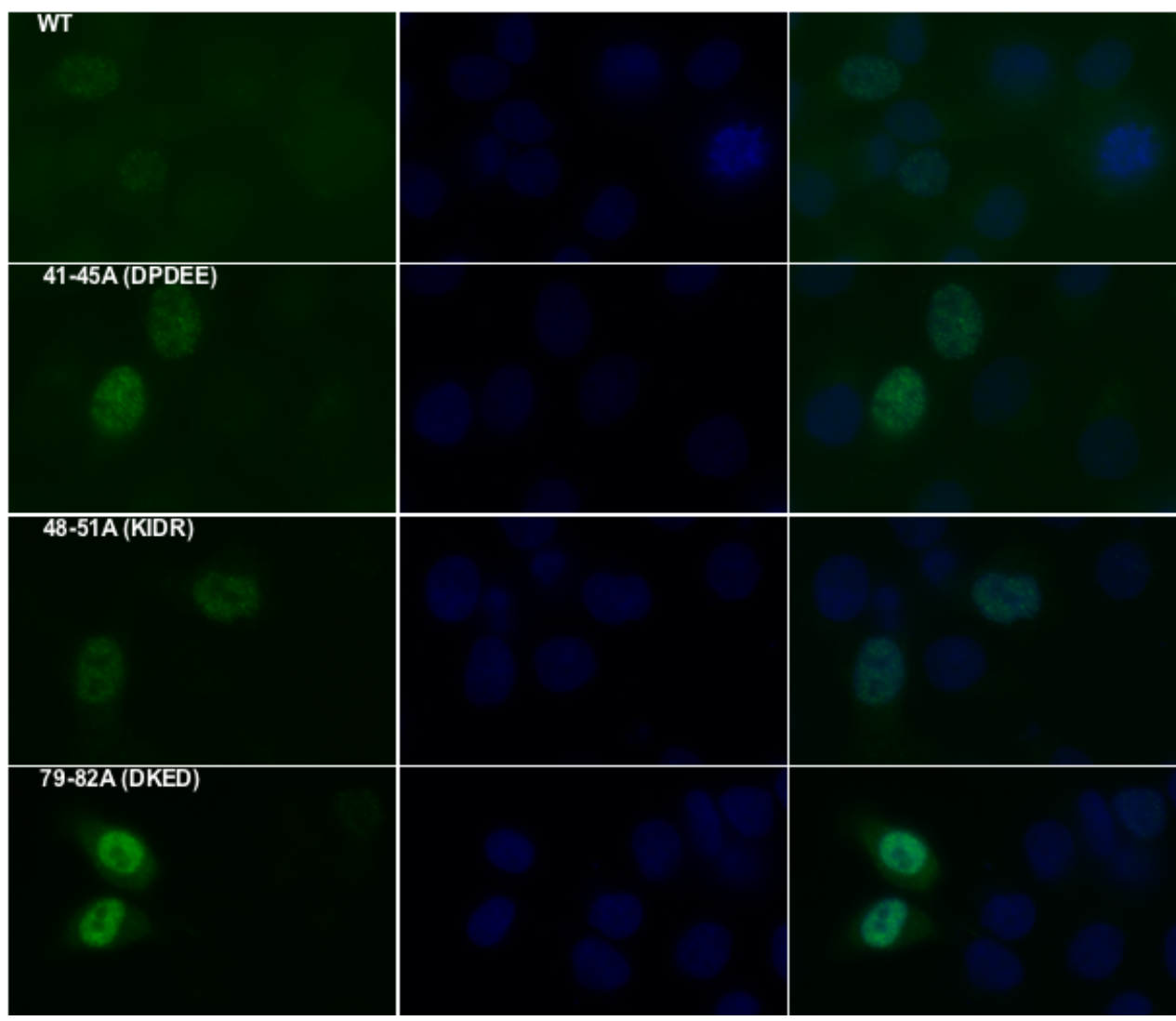

Figure 2.5: Localization of cyclin E mutants resembles wild-type. Myctagged cyclin E mutants were transfected into HeLa cells and localization was determined using immunofluorescence. The localization of several mutants is shown: DPDEE $\rightarrow$ AAAAA (row 2), KIDR $\rightarrow$ AAAA (row 3), and DKED $\rightarrow$ AAAA (row 4). The top row shows the localization of wild-type cyclin $\mathrm{E}$. 


\section{Lysine 48 on cyclin E serves as a ubiquitination site for Cul3}

To pinpoint specific residues on cyclin E that may be involved in degradation, cyclin E alanine scanning mutants were transfected into the Cul3 WT and KO 293 cells. Three alanine-scanning mutants, DPDEE $\rightarrow$ AAAAA (amino acids 41-45), KIDR $\rightarrow$ AAAA (amino acids 48-51), and DKED $\rightarrow$ AAAA (amino acids 79-82), appear more stable in the wild-type cells, which suggests that they are less likely degraded by Cul3 (Figurer 2.4C). All three of these mutants are located near the $\mathrm{N}$-terminus of cyclin $\mathrm{E}$ and in order to further describe the importance of this region for Cul3mediated degradation to occur, two constructs containing deletions in this region were created (Figure 2.4D). The first construct, cyclin E $\Delta 31-82$, is missing the region containing all three of the stabilized alanine scanning mutants. The second construct, cyclin E $\Delta 2-86$, resembles the LMW cyclin E found in some cancer cells, and is missing the entire N-terminal region (Figure 2.4D). Both of these mutants were transfected into the wild-type and Cul3 KO HEK293 cells, and both demonstrated increased stability in the WT cells (Figure 2.4E, compare lane 1 to 2, and lane 3 to lane 4), suggesting that this region may be necessary for Cul3mediated degradation of cyclin E to occur.

Sometimes, phenotypes exhibited by mutant proteins can be explained by changes in the localization of the protein within the cell. In order to determine if the phenotypes of the three stable alanine-scanning mutants are the result of localization changes, HeLa cells were transfected with each construct and visualized using immunofluorescent microscopy. The cellular localization of the three mutants was found to be predominantly nuclear, similar to wild-type cyclin E, indicating that 
their increased stability was not a result of a change in cellular localization (Figure 2.5). The DPDEE $\rightarrow$ AAAAA (residues 41-45) mutant cannot bind Cul3 as well as the others (Figure 2.3), which provides an explanation for its increased stability in our assay. As the KIDR $\rightarrow$ AAAA (residues 48-51) and DKED $\rightarrow$ AAAA (residues 79-82) mutants both contain lysine residues and are located near the N-terminus of cyclin E (Figure 2.4C). The increased stability of the KIDR and DKED alanine scanning mutants as well as the deletion mutants imply that both lysine residues K48 and K80 are potential ubiquitination sites, and the degron that is recognized by Cul3 likely resides within the $\mathrm{N}$-terminal portion of cyclin $\mathrm{E}$.

To establish if ubiquitination on K48 or K80 of cyclin E regulates its stability, we determined if the KIDR $\rightarrow$ AAAA mutant or DKED $\rightarrow$ AAAA mutant stabilized by a mutation in ubiquitin that prevents $\mathrm{K} 48$ branching, a type of branching that leads to degradation (Grice and Nathan 2016). To test this, all of the alanine scanning mutants within the first 200 amino acids of cyclin E were transfected into both cell types in the presence or absence of a mutant K48R ubiquitin. We observed that, unlike WT cyclin E which was stabilized in WT cells by the addition of the dominantnegative K48R ubiquitin mutant (Figure 2.6 compare lanes 1 and 2 to lanes 3 and 4), the KIDR $\rightarrow$ AAAA mutant was unaffected indicating it is no longer a substrate for ubiquitination-dependent degradation (Figure 2.6 lanes13-16). The DPDEE $\rightarrow$ AAAAA (Cul3 binding mutant, lanes 9-12) mutant behaved similarly to the KIDR $\rightarrow$ AAAA mutant, which suggests that they might comprise a Cul3 degron, which consists of a binding region (DPDEE) and ubiquitination site (K48 on cyclin E, the lysine contained in the KIDR mutant). The DKED $\rightarrow$ AAAA mutant (Figure 2.6 lanes 43 
17-20) appears similarly to wild-type (Figure 2.6 lanes 1-4) in this assay suggesting the K48 (the KIDR lysine) and not K80 (the DKED lysine) is the ubiquitination site for Cul3. As a second control, cyclin E T395A, which cannot be degraded by Cul1, was examined and we observed that it is a substrate of Cul3 (Figure 2.6 lanes 5 through 8). In order to confirm that $\mathrm{K} 48$ is an ubiquitination site on cyclin $\mathrm{E}$, a point mutant, cyclin E K48R, was constructed for use in the ubiquitination assay. Like the KIDR mutant, cyclin E K48R appears relatively stable (Figure 2.6 lanes 21-24). The increased stability of the KIDR mutant and cyclin E K48R suggests that K48 on cyclin $\mathrm{E}$ is indeed an ubiquitination site utilized by Cul3 in vivo. Taken together, these data suggest that the residues DPDEE (binding site) and KIDR (ubiquitination site) are part of a degron that is recognized by Cul3.

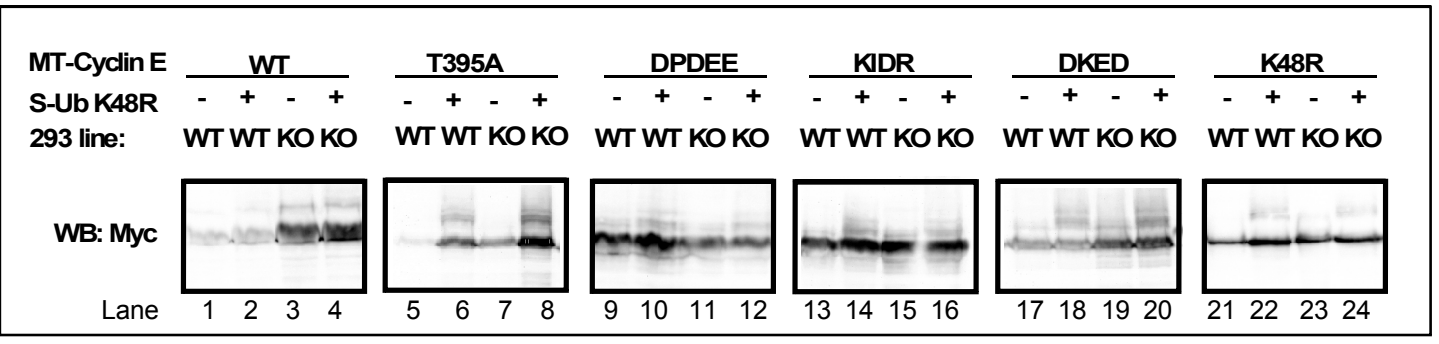

Figure 2.6: $K 48$ is a Cul3 ubiquitination site on cyclin $E$. Alanine scanning and point mutants located within the first 200 amino acids of the cyclin E protein were transfected in WT and KO 293 cells with or without the addition of an S-tagged K48R ubiquitin construct. Wild-type cyclin E (lanes 1-4) and cyclin E T395A, which cannot be degraded by Cul1 (lanes 5-8), were included as controls. Three cyclin E alanine scanning mutants are shown here, DPDEE $\rightarrow$ AAAAA (residues 41-45, lanes 912 ), KIDR $\rightarrow$ AAAA (residues 48-51, lanes 13-16), and DKED $\rightarrow$ AAAA (residues 79-82, lanes 17-20). The point mutant cyclin E K48R is also shown (lanes 21-24). 


\section{The $\mathrm{N}$-terminal domain of cyclin $\mathrm{E}$ is required for degradation}

In order to truly measure if cyclin $\mathrm{E}$ that lacks its $\mathrm{N}$-terminus is differentially degraded by Cul3 compared to wild-type cyclin E, it is necessary to measure the rate of degradation of these proteins. To ascertain the role of the N-terminal domain of cyclin E degradation, cyclin E half-lives were measured and compared in cells of both genotypes (Cul3 WT and KO). Cells were transfected with full-length Myctagged cyclin E or Myc-tagged cyclin E $\Delta 2-86$. Following addition of cycloheximide (CHX) the cells were harvested every two hours for ten hours. We observed that WT cyclin E has a half-life of about 2 hours in the WT cell line but a half-life of 5 hours in the KO cells demonstrating that loss of Cul3 reduces the half-life of cyclin E (Figure 2.7 compare WT cyclin E (left) to cyclin E $\Delta 2-86$ (right). We also observed that cyclin E $\Delta 2-86$ has a 5 hour half-life and shows equal stability in both WT and Cul3 KO cells, demonstrating the inability of Cul3 to target it for degradation (Figure 2.7, right panel, lanes 1-5 vs. lanes 6-10). 

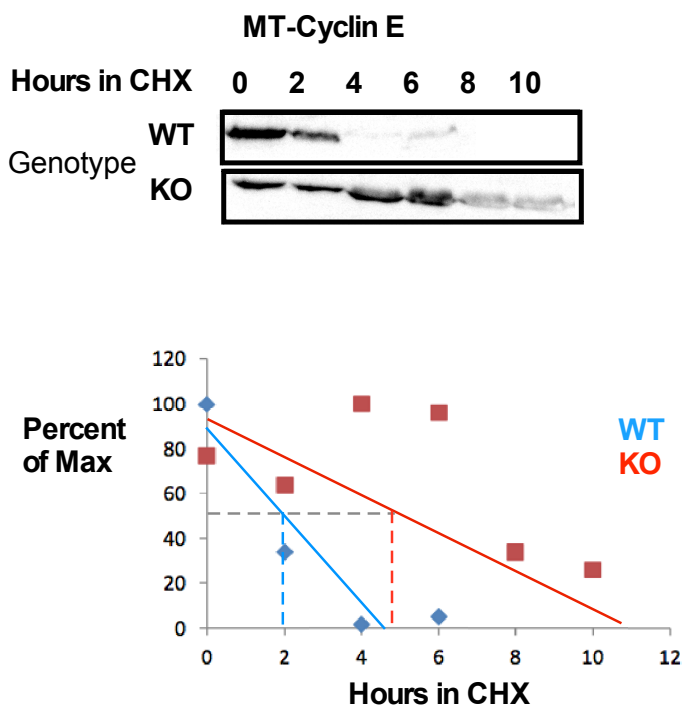

MT-Cyclin E $\Delta 2-86$
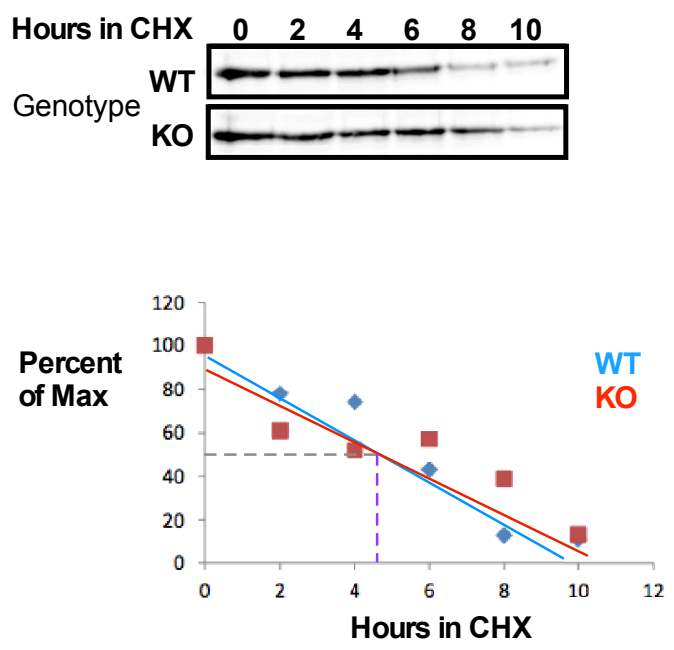

Figure 2.7: The N-terminal domain of cyclin $E$ is required for Cul3-mediated degradation. Cul3 WT and KO 293 cells were transfected with Myc-tagged cyclin E (Left) or Myc-tagged cyclin E $\Delta 2-86$ (Right). After 24 hours, cycloheximide was added and cells were harvested at the indicated time points. Half-lives were determined via Western blots (top), and quantified (bottom). Repetition of this experiment yields similar results.

\section{Cul3 cannot degrade endogenous LMW cyclin E}

As low molecular weight (LMW) cyclin E can be found endogenously in some cells, we sought to determine if loss of Cul3 affects the presence of these LMW forms. It has been previously shown that the LMW cyclin E truncations can still be degraded in a Cul1-dependent manner (Delk et al. 2009). Overall, the Cul3 KO 293 cells have more endogenous cyclin E than the wild-type (Ibeawuchi et al. 2015). We observed that the $50 \mathrm{kDa}$ endogenous cyclin $\mathrm{E}$ band increases upon inhibition of the proteasome in WT cells to equal the amount of the $50 \mathrm{kDa}$ protein that is present in the $\mathrm{KO}$ cells prior to proteasome inhibition, demonstrating that the $50 \mathrm{kDa}$ band is a 
substrate of Cul3 in 293 cells (Figure 2.8 lane 1 vs lane 3). Two LMW cyclin E bands are also detected, the smallest of which is about $43 \mathrm{kDa}$, and they appear to be equally expressed in WT and KO cells (Figure 2.8 lanes 1 and 3). The relative abundance of the LMW bands is elevated equally in both cell types upon proteasome inhibition (Figure 2.8 lanes 2 and 4), indicating that LMW cyclin E still targeted for ubiquitin-mediated proteolysis equally in both cell types.

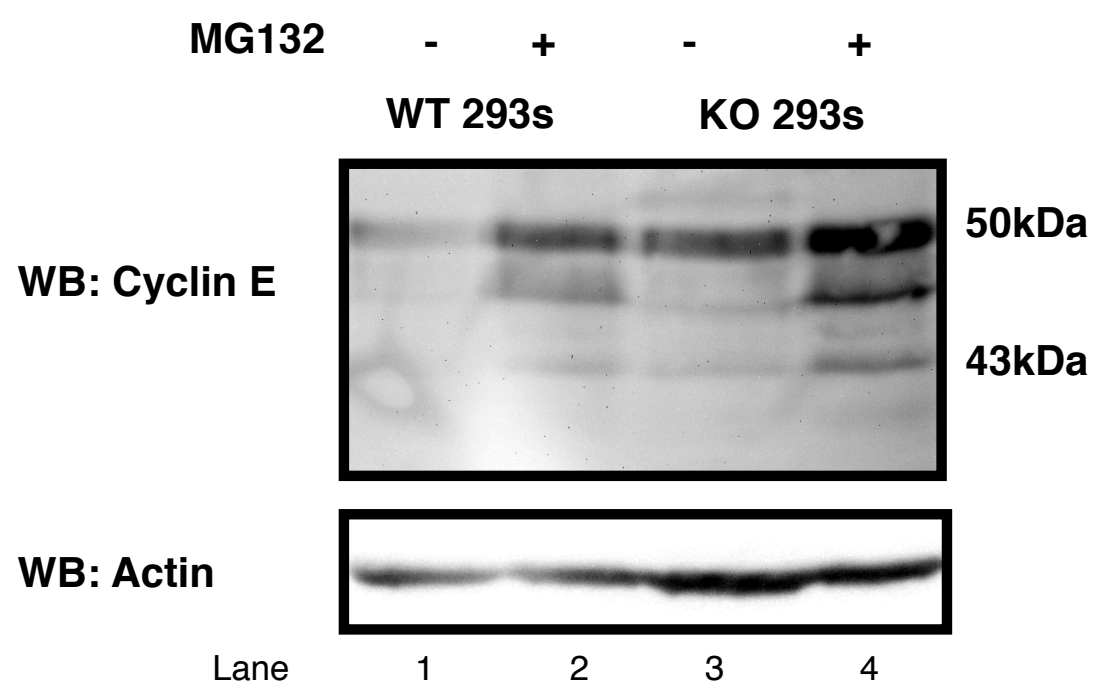

Figure 2.8: $\quad L M W$ cyclin $E$ is not degraded by Cul3. The upper blot shows levels of endogenous cyclin E in WT and Cul3 knockout (KO) 293 cells. The proteasome inhibitor MG132 has been added to the cells shown in lanes 2 and 4 . The lower blot shows levels of actin in the same cells.

\section{Cyclin E2 is not a substrate of Cul3}

Mammals contain two cyclin E genes, CCNE1 (cyclin E1) and CCNE2 (cyclin E2), which produce different proteins (Sherr and Roberts 1999; Geng et al. 2003). The two cyclin E proteins share high homology and many structural similarities, including the Cdk2 interacting domain (Perez-Neut et al. 2015). In order to determine if the observed degradation of cyclin E was specific to cyclin E1, cyclin E2 
was transfected into the WT and KO 293 cells. Cyclin E2 is evenly expressed in both cell types, indicating that it does not get ubiquitinated by Cul3 (Figure 2.9, lanes 1 and 3 compared to lanes 2 and 4 ).

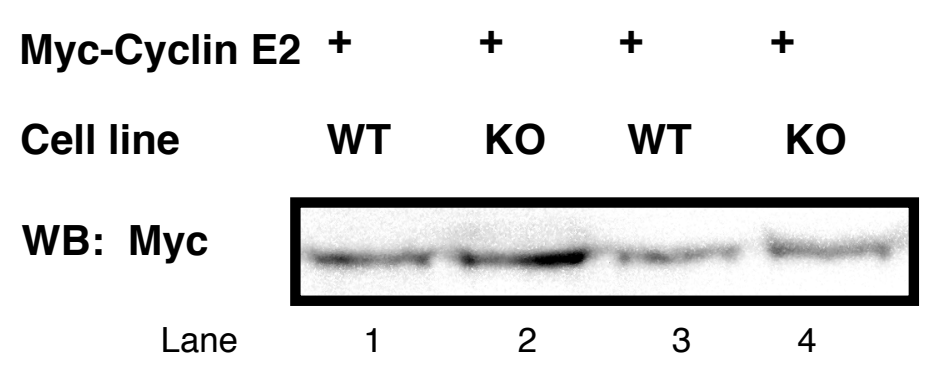

Figure 2.9: Cyclin E2 is not a Cul3 substrate. Transfected, Myc-tagged cyclin E2, shown here in duplicate is shown here in WT (Lanes 1 and 3) and Cul3 KO (lanes 2 and 4) HEK 293 cells.

\section{Loss of Cul3 results in early accumulation of cyclin E after release from serum starvation}

In order to determine when loss of Cul3 resulted in cyclin $\mathrm{E}$ accumulation we turned to the Cul3 knockout 293 cells. Previous work from our lab has shown that loss of Cul3 in mouse embryonic fibroblasts (MEFs) results in increased amounts of cyclin E and a greater percentage of cells in S-phase (McEvoy et al. 2007). Analysis of Cul3 KO 293 cells by flow cytometry shows an increased percentage of cells in S phase when compared to WT 293s (Figure 2.10). This result shows that the excess cyclin E in the Cul3 KO 293 cells is functional as it is capable of initiating DNA replication. To determine at which point during the cell cycle the Cul3 KO cells begin to accumulate excess cyclin E, both WT and $\mathrm{KO}$ cells were arrested in $\mathrm{G}_{1}$ via serum starvation and released. The KO cells enter S-phase earlier than their wild- 
type counterparts, as four hours after release into $\mathrm{G}_{1}$, only 47.1 percent of wild-type cells are in S-phase or G2 but 61.7 percent of Cul3 KO cells have reached this point (Figure 2.11, WT cells are shown on the left and KO are shown on the right).

In order to determine if the increased percentage of cells in S-phase is a result of cyclin $E$ accumulation during $\mathrm{G}_{1}$, we utilized a similar serum-starve and release experiment. We examined cyclin E accumulation in Cul3 hypomorphic MEFs (mouse embryonic fibroblasts) after serum starvation and release. We observed cyclin E accumulation 2 hours earlier in Cul3 hypomorphic (floxed) MEFs than in WT MEFs (Figure 2.12 WT top row, Cul3 hypomorphic flx/flx bottom row). This is consistent with a role for Cul3 in regulation of cyclin E levels in quiescent cells, as we had observed in an animal model (McEvoy et al. 2007), and indicates Cul3 regulation of cyclin $\mathrm{E}$ occurs in a different temporal window than the Cul1 regulation of cyclin $\mathrm{E}$ which takes place 4 hours after the onset of DNA replication (Bhaskaran et al. 2013). 

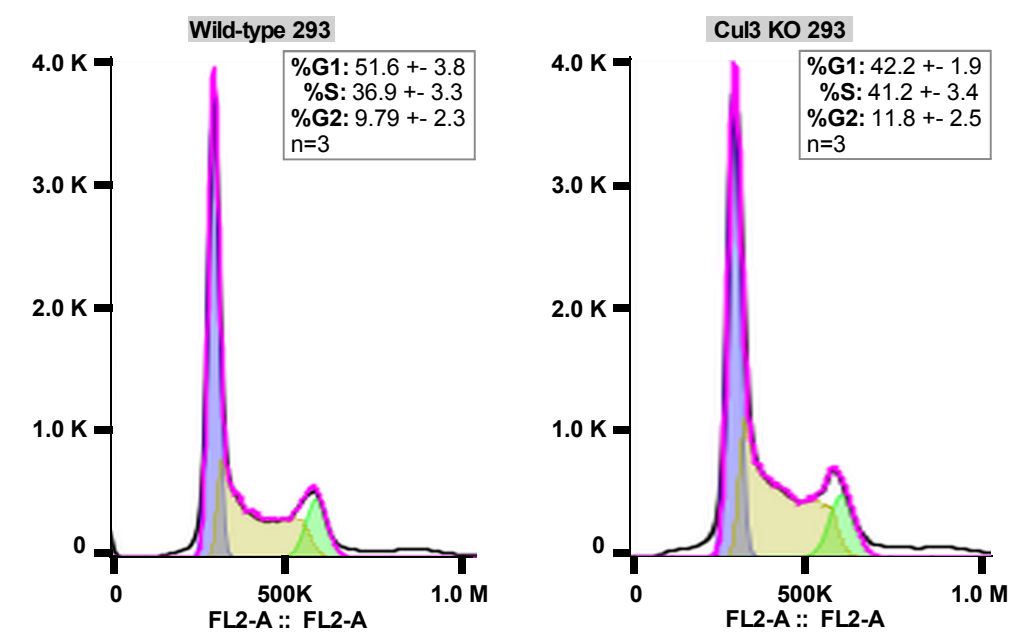

Figure 2.10: The excess cyclin E in Cul3 KO 293 cells increases proliferation. Flow cytometry analysis of proliferating 293 cells stained with propidium iodide, which labels the DNA allowing for the quantification of DNA content. WT cells are shown on the left and Cul3 KO cells are shown on the right. Cells in $\mathrm{G}_{1}$ are shown in purple, $\mathrm{S}$-phase cells are shown in yellow, and $\mathrm{G}_{2} / \mathrm{M}$ cells are shown in green. At least 20,000 cells were counted for each experiment. The quantification show represents and average of three experiments. 


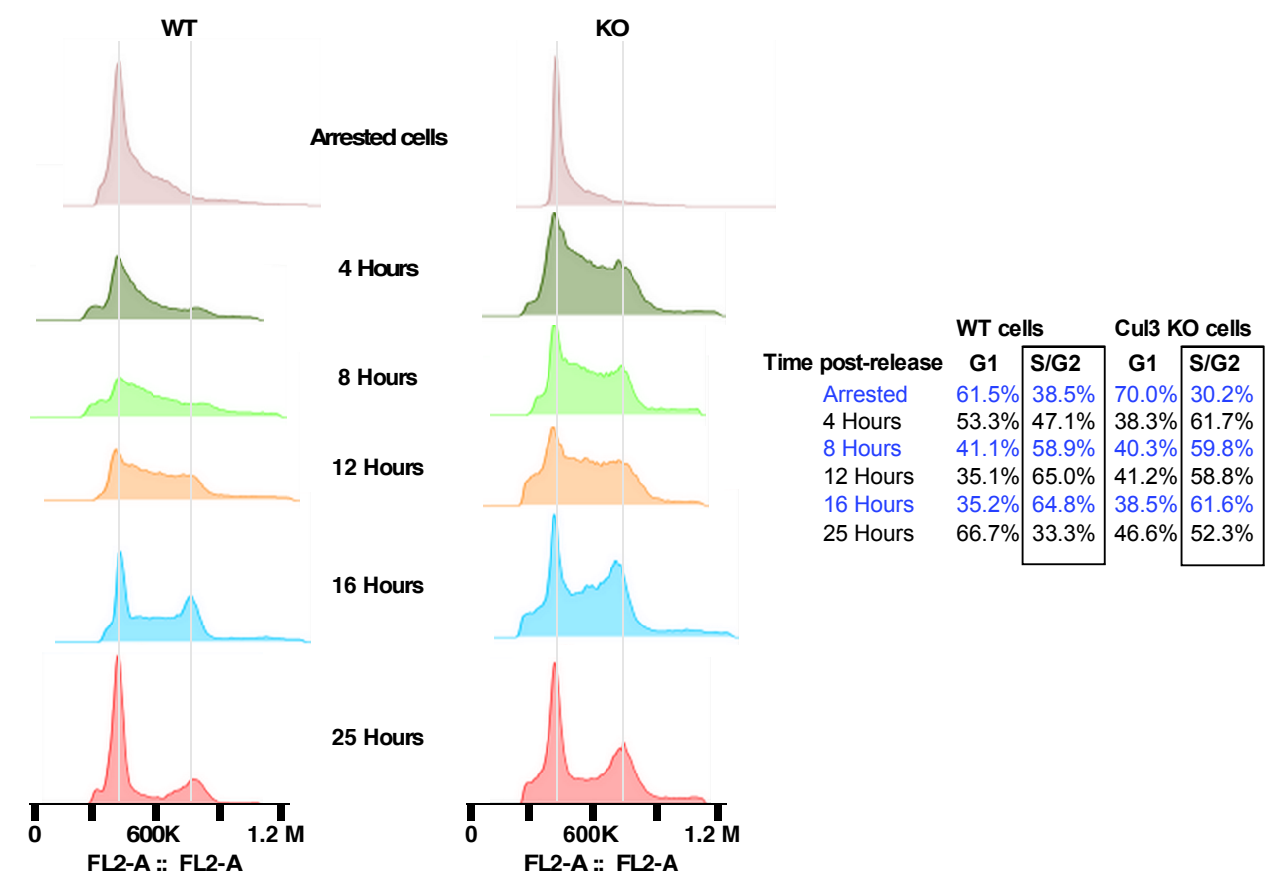

Figure 2.11: Cells lacking Cul3 enter S-phase early. Cells were serum-starved and released into $\mathrm{G}_{1}$. Cells were harvested at 4-hour intervals, stained with propidium iodide, and analyzed by flow cytometry. Quantification of the resulting cell cycle profiles is shown (Right).

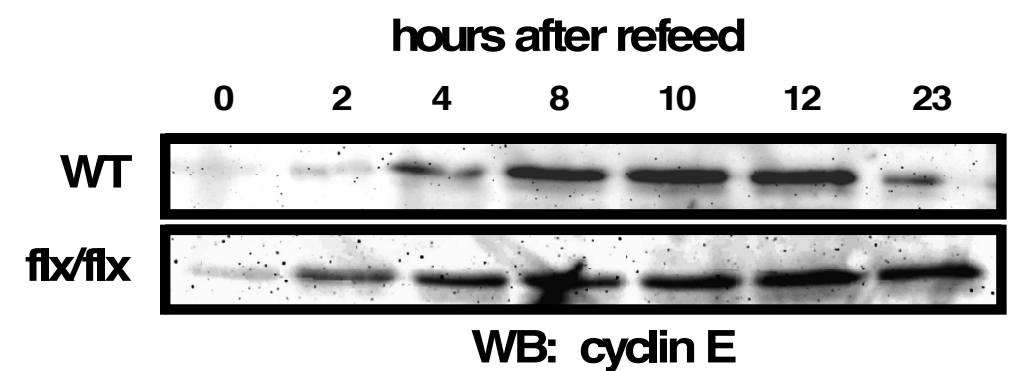

Figure 2.12: Cyclin E levels increase early in cells that are hypomorphic for Cul3. Western blot showing levels of endogenous cyclin $\mathrm{E}$ in mouse embryonic fibroblasts (MEFs) that are wild-type (WT, top row) or deficient for Cul3 (flx/flx, bottom row). Cells that had been serum-starved and released were collected at two-hour intervals as indicated. 


\section{RhoBTB3 interacts with UbE2E1 to facilitate complex formation}

The previous data imply that cyclin E can bind Cul3 without the assistance of a BTB domain-containing substrate adaptor such as RhoBTB3. We therefore became interested in what potential role RhoBTB3 had in Cul3 mediated cyclin E degradation, as it has been suggested that RhoBTB3 facilitates Cul3-mediated ubiquitination of cyclin E (Lu and Pfeffer 2013). Like Cul3, RhoBTB3 binds to the cyclin E STOP 200 and STOP 300 truncations (Figure 2.13 panel A lanes 2 and 4). RhoBTB3 shows a decreased ability to bind the DPDEE $\rightarrow$ AAAAA (amino acids 4145) alanine scanning mutant in comparison to other mutants tested, which is also similar to Cul3 (Figure 2.14 panel B, compare lane 6 to lanes 2 and 4). Binding of RhoBTB3 to the DKED $\rightarrow$ AAAA (amino acids 79-82) alanine scanning mutant also appears reduced (Figure 2.13 lane 8).

Since RhoBTB3 is not essential for the Cul3/cyclin E interaction, we speculated that it might be playing another role in the active complex. We hypothesized that since specific E2 recruitment should be an essential role for the E3 ligase it may be that RhoBTB3 is responsible for recruiting the E2 enzyme that ubiquitinates cyclin E. If BTB proteins are involved in E2 selection, it would ensure that the proper E2 is recruited to form the correct ubiquitin linkage for a given substrate. To test this hypothesis, we examined the binding of Cul3 and RhoBTB3 to the E2 enzyme UbE2E1 which forms degradative K48-linked chains and has been shown to associate with Cul3 (Plafker et al. 2009). We observed that both Cul3 and RhoBTB3 bind to UbE2E1 (Figures 2.14 lane 2 and 2.15 lane 2). We then examined if Cul3 binding to UbE2E1 requires the BTB domain interacting region on Cul3. We 
observed that UbE2E1 cannot bind the Cul3 mutant that is incapable of binding BTB proteins, Cul3 $\Delta 51-67$ (Figure 2.15 lane 3). This indicates that the interaction with the E2 enzyme is likely not entirely mediated by RhoBTB3. To confirm that Cul3 does not mediate the interaction between RhoBTB3 and UbE2E1, Cul3 KO 293 cells were used to analyze binding (Ibeawuchi et al. 2015). As can be seen in lanes two and five, RhoBTB3 binds to the E2 enzyme in the absence of Cul3 (Figure 2.14 lane 4) to the same degree that the two proteins bind in the presence of Cul3 (Figure 2.14 lane 2) indicating that Cul3 is not required for the BTB to interact with the E2 and BTB proteins may be necessary to help associate the E2 enzyme with Cul3. Together these results demonstrate that a BTB protein, in this instance RhoBTB3, can interact with an E2 enzyme independently of Cul3 and may enhance E2 binding in vivo. 


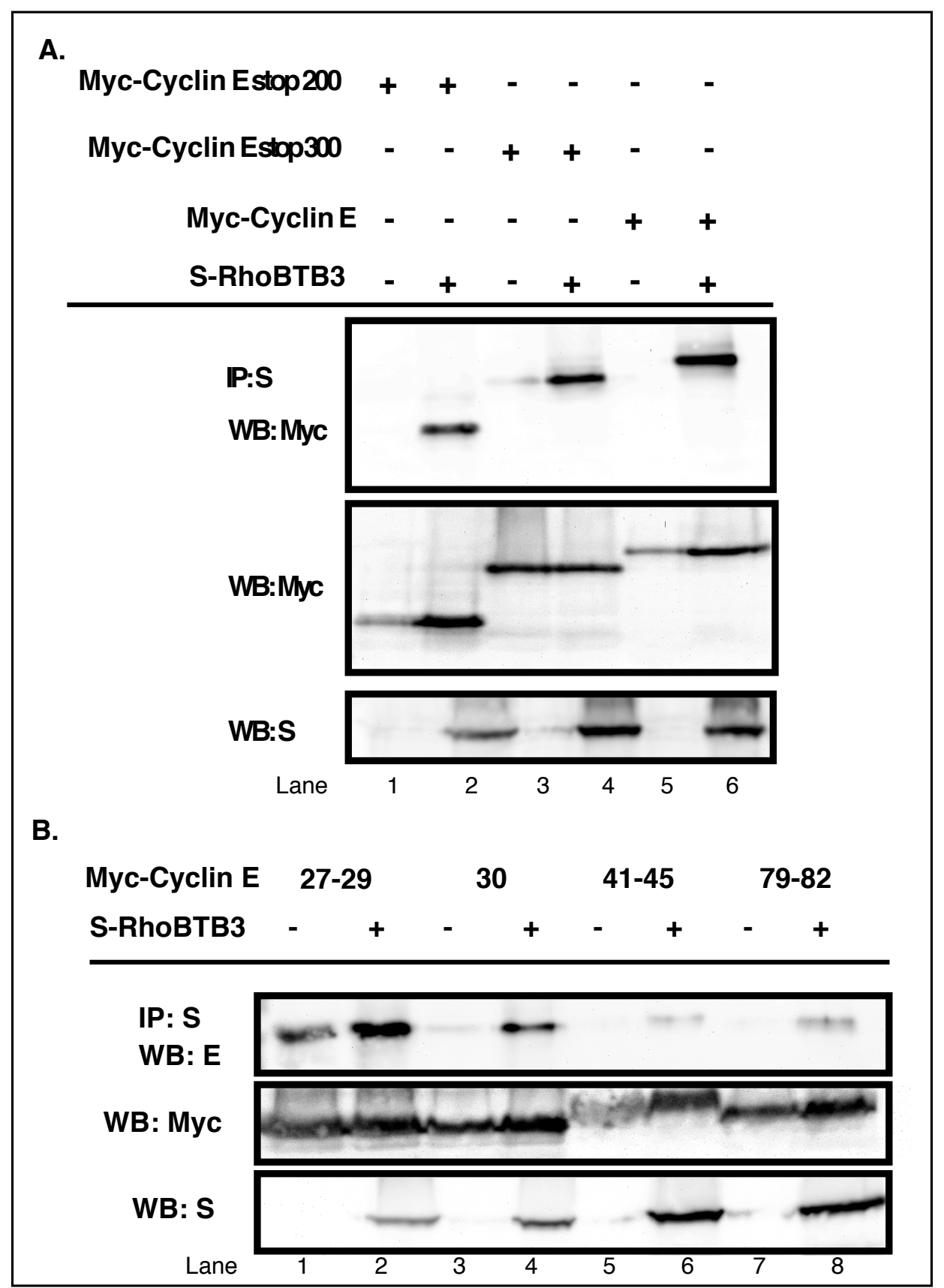

Figure 2.13: RhoBTB3 interacts with the $N$ terminus of cyclin $E$. A: Upper blot, immunoprecipitation results showing binding between S-tagged RhoBTB3 and Myctagged cyclin E truncations. The lower blots show levels of the transfected proteins in the cell extracts. B: The upper blot shows immunoprecipitations results to check for binding between S-RhoBTB3 and Myc-cyclin E alanine scanning mutants. The Four mutants shown here are 27-29 (RSR, lanes 1 and 2), 30-32 (KRK, lanes 3 and 4), 41-45 (DPDEE, lanes 5 and 6), and 79-82 (DKED, lanes 7 and 8). The lower blots show the expression of the transfected proteins in cell lysates. 


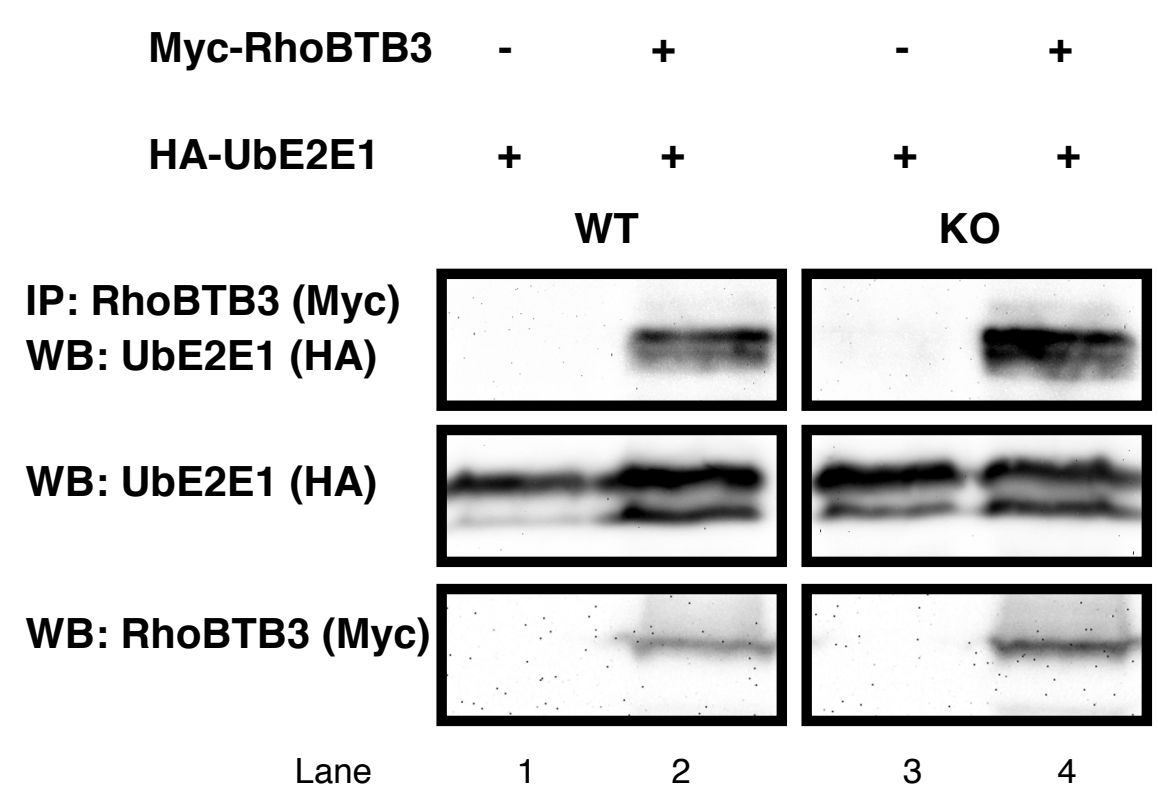

Figure 2.14: RhoBTB3 binds an E2 enzyme. RhoBTB3 was co-transfected with the E2 ubiquitin conjugating enzyme HA-tagged UbE2E1 in the presence of endogenous Cul3 (WT cells, lanes one and two) and absence of Cul3 (KO cells, lanes three and four). Immunopreciptations (IPs) were used to check binding.

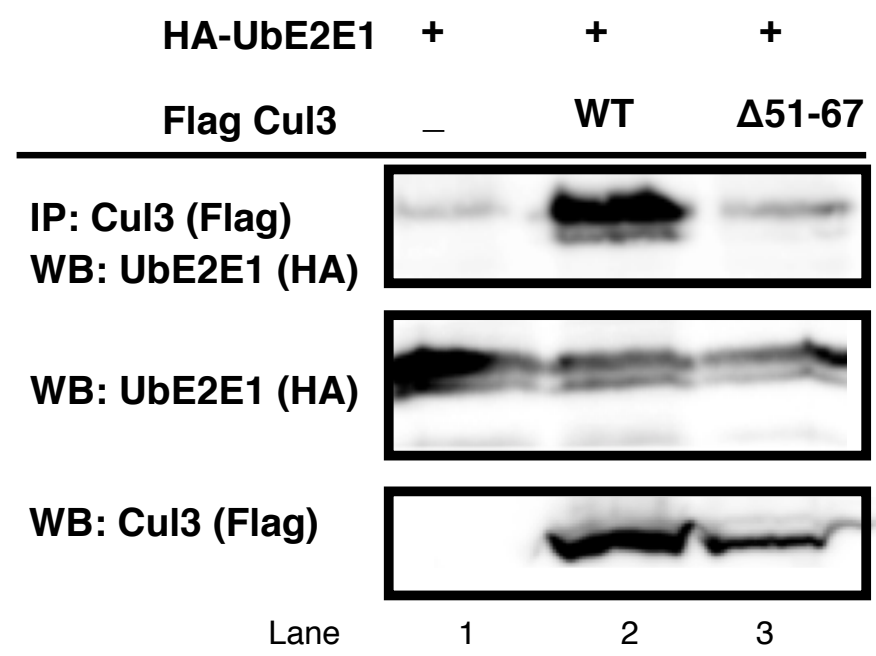

Figure 2.15: Cul3 requires a BTB protein to interact with UbE2E1. Flag-WT Cul3 (lane 2) and Flag-Cul3 $\Delta 51-67$, which cannot bind BTB proteins, (lane 3) were cotransfected with the HA-tagged E2 enzyme UbE2E1. Immunoprecipitations using Flag antibody were conducted to check for binding. The top row shows the IP results while the bottom two rows show the protein expression in the cell extracts. 


\section{DISCUSSION:}

Cyclin E protein accumulates in late $\mathrm{G}_{1}$, peaks in early S-phase and rapidly disappears. Increased levels of cyclin E are associated with cell cycle errors and tumorigenesis and loss of cyclin E in MEFs results in the inability of the cells to exit from quiescence (Said and Medina 1995; Geng et al. 2003). Cyclin E undergoes several post-translational modifications in cells including phosphorylation and ubiquitination resulting in its degradation by the proteasome. Research has shown that cyclin E can be proteolytically cleaved resulting in truncated forms of the cyclin, many of which lack portions of the protein near the N-terminus (Porter et al. 2001; Wang et al. 2003). These LMW forms of cyclin E are associated with poor prognosis in cancer patients and overexpression of the LMW cyclin E has also been shown to cause increased cell cycle errors and chromosome instability in cell culture models compared to full length cyclin E (Bagheri-Yarmand et al. 2010). Others have shown that the LMW forms of cyclin E have an increased ability to activate Cdk2, resulting in an increase of Cdk2 kinase activity (Porter et al. 2001; Wingate et al. 2005). It has also been demonstrated that these cyclin E truncations can be degraded in a ubiquitin-dependent manner (Delk et al. 2009).

Two ubiquitin ligase pathways, Cul1 and Cul3, are known to degrade cyclin E. Cul1-mediated degradation has been extensively investigated and it is known to degrade cyclin E that is bound to Cdk2 and require phosphorylation of residues T77 and T395 on cyclin E in order to recognize it for degradation (Clurman et al. 1996; Loeb et al. 2005; Minella et al. 2008). In contrast, the Cul3-based pathway is not as well understood and is known to degrade cyclin E that is not bound to Cdk2 (Singer 
et al. 1999). LMW cyclin E containing complexes have been shown to be more poorly inhibited by the cyclin-dependent kinase inhibitors (CKIs) p21 and p27 (Akli et al. 2004; Wingate et al. 2005). It has been proposed that the LMW cyclin E may be capable of sequestering the CKIs and therefore preventing them from interacting with full length cyclin E, which is believed to be more susceptible to inhibition, ultimately resulting in the increased Cdk2 activity that is associated with the LMW cyclin E (Wingate et al. 2005). Our data implies that decreased inhibition of LMW cyclin E by p27 and p21 may only provide a partial explanation for the accumulation of LMW cyclin E and a secondary Cul3-based mechanism may also contribute to the increased activity. This mechanism results from bypassing Cul3 degradation of cyclin $\mathrm{E}$ by cleaving off the $\mathrm{N}$-terminal degron of cyclin $\mathrm{E}$ making more available to interact with Cdk2 (Figure 2.16).

We show that K48 is a ubiquitination site on cyclin E utilized by Cul3 (Figure 2.6). This finding is notable given what is known about the structure of the cyclin $E$ protein as the $\mathrm{N}$-terminus, which contains $\mathrm{K} 48$, is believed to be a disordered, mostly hydrophilic region (Rath and Senapati 2014). The structure of cyclin E bound to Cdk2 has been determined (Honda et al. 2005), but the published structure only includes amino acids 81-363 of cyclin E and therefore did not include the Nterminus of the cyclin. Recently, advanced modeling techniques have predicted a structure for the N-terminal region of cyclin E which shows that this portion of the protein is mostly disordered with the notable exception of a predicted alpha helix encompassing the sequence "DEEMAKID" (amino acids 43-50, Figure 2.18A) (Rath and Senapati 2014). Coincidentally, the alanine scanning mutants in this region 
(DPDEE $\rightarrow$ AAAAA and KIDR $\rightarrow$ AAAA) exhibited the most notable phenotypes in our study as DPDEE exhibited diminished binding to the Cul3 complex and KIDR $\rightarrow$ AAAA cannot be degraded in a Cul3-dependent manner (Figures 2.3, 2.4 and 2.6). Our data suggests that this structural motif may form part of the interface on cyclin E, which is responsible for Cul3 interaction and also functions as the Cul3 degron on cyclin E (Figure 2.17A). The putative helix comprising the Cul3 degron, including the K48 ubiquitination site, is conserved in other mammalian species (Figure 2.17B), which is notable as Cul3 also regulates cyclin E in mice (Singer et al. 1999; McEvoy et al. 2007). Additionally, cyclin E2, which cannot be degraded by Cul3 (Figure 2.9), does not resemble cyclin E1 in the region of the newly proposed degron (figure 2.17C). The net charge of the region was calculated by finding the difference between the number of basic residues and the number of acidic residues. Using this method, it can be seen that cyclin E1 is very acidic in this region with a net charge of negative two. Cyclin E2, in contrast, is extremely basic with a net charge of four (Figure 2.17C). Additionally, a Chou-Fasman algorithm was used to predict possible structures of both cyclin E proteins in the degron region (Chou and Fasman 1975). This method revealed what is possibly a helical region in cyclin E1 but an unstructured region in cyclin E2 (Figure 2.17C). Taken together, this information suggests that the Cul3 degron comprises a structural feature that is unique to cyclin E1. These data demonstrate the critical role of the Cul3 complex in degradation of cyclin E by providing evidence that suggests that Cul3's ability to degrade LMW cyclin E which lack their N-termini may be impaired as the Cul3 degron is located near the cyclin E N-terminus. Cul3 uses a mechanism distinct from the Cul1-based 
pathway to recognize cyclin E for degradation, as Cul3 does not require cyclin E to be phosphorylated on T395 for degradation to occur (Figure 2.6 lanes 5-8).

The data presented here support the idea that LMW cyclin E is still a substrate of the Cul1 ubiquitin ligase pathway since LMW cyclin E increases in the Cul3 KO 293 cells upon the addition of MG132, indicating that it is still degraded in a ubiquitin-dependent manner (Figure 2.8). In addition, other labs have also shown that LMW cyclin E can still be degraded by the ubiquitin proteasome system (Delk et al. 2009).

It has been suggested that Cul3 utilizes the substrate adaptor RhoBTB3 to target cyclin E for degradation (Lu and Pfeffer 2013). Our work sheds light on the mechanistic purpose of RhoBTB3 in cyclin E degradation as we demonstrate that RhoBTB3 is involved in the binding of the E2 ubiquitin conjugating enzyme and can bind the E2 UbE2E1 both in the presence and absence of Cul3 (Figure 2.1). This finding is significant as it suggests that Cul3 substrate adaptors may play a role in E2 selection, which would help to explain how Cul3 is capable of forming a variety of ubiquitin chain linkages as different E2s are associated with this process. The revelation that Cul3 $\Delta 51-67$, which cannot bind BTB proteins, is incapable of binding UbE2E1 (Figure 2.15 lane 3 compared to lane 2) presents a second piece of information in support of this idea that BTB proteins are necessary for E2 selection to occur. These findings may shed light on interactions between Cul3, BTB proteins, and E2s, a finding which may be applicable to other BTB proteins and substrates. In addition to clarifying the mechanistic details regarding Cul3-mediated degradation of cyclin E, the work presented here also identifies the temporal window during 59 
which such degradation occurs. Previous work has shown that loss of Cul3 results in cyclin E accumulation and exit from quiescence in mice (McEvoy et al. 2007), leading us to hypothesize that loss of Cul3 results in earlier increases in cyclin $\mathrm{E}$ and entry into S-phase. Here, this hypothesis is supported as cells that are hypomorphic for Cul3 both enter the cell cycle earlier than their WT counterparts and show increased levels of cyclin E earlier than wild-type cells following release from quiescence (Figure 2.11 and 2.12). These data suggest that Cul3 is responsible for maintaining levels of cyclin E earlier in the cell cycle and preceding the start of Sphase, which is again in contrast to the Cul1 degradation pathway that degrades cyclin E later during S-phase (Bhaskaran et al. 2013). Taken together, our data suggests that during G1, Cul3 functions to suppress levels of cyclin E via ubiquitination of its $\mathrm{N}$-terminal domain (Figure 2.18). Lack of cyclin E regulation by Cul3 during G1 might contribute to the increased cyclin E/Cdk2 activity that is observed in LMW cyclin E containing cancer cells (Figures 2.16 and 2.18). 

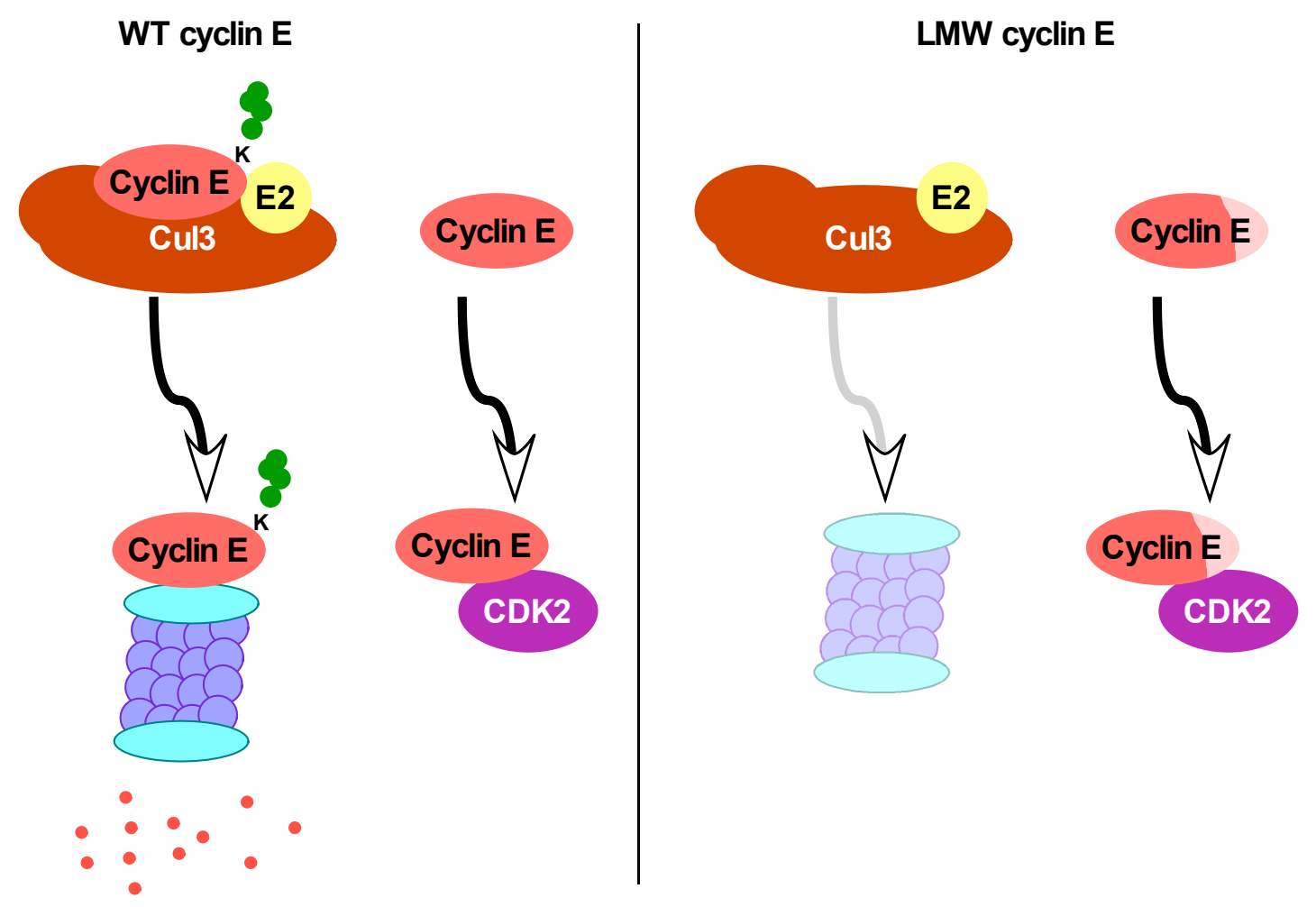

Figure 2.16: LMW cyclin E cannot be degraded by Cul3. This model demonstrates Cul3's role in degradation of full-length cyclin E (Left) and contrasts it with improper regulation of LMW cyclin E (Right). Full length cyclin E is ubiquitinated and degraded via Cul3 during $\mathrm{G}_{1}$, leaving some cyclin $\mathrm{E}$ available to activate Cdk2. However, in cells containing LMW cyclin E which lacks its N-terminal domain, proper degradation of LMW cyclin E by Cul3 might not occur, which might result in increased activation of Cdk2. 


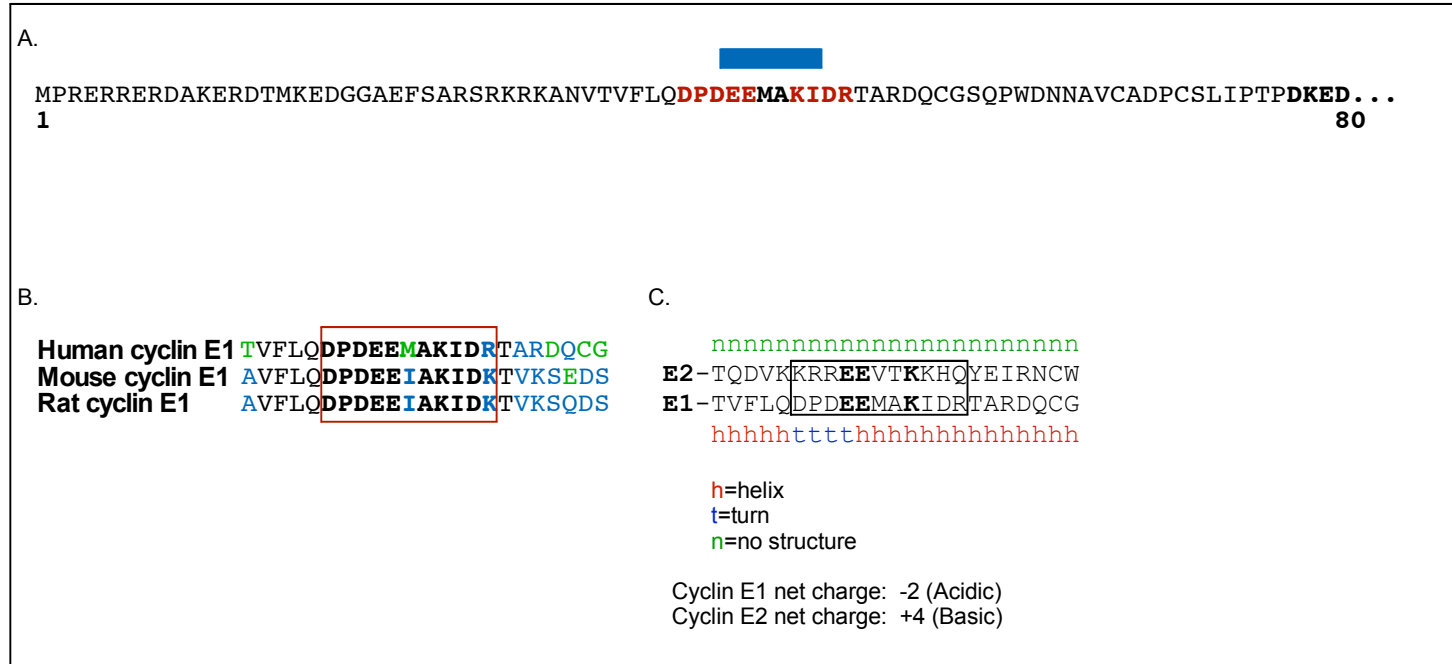

Figure 2.17: Cyclin E1 contains a putative Cul3 degron that is conserved in other mammals but lacking in cyclin E2. A: Others have proposed a helix located in the Nterminal region of cyclin E1 (Rath and Senapati, 2014). The proposed helix (A, shown in blue) overlaps the DPDEE (Cul3 binding) and KIDR (ubiquitination site) alanine scanning mutants (shown in red), suggesting that the Cul3 degron is part of a structural feature on cyclin E. B: The Cul3 degron is highly conserved in mouse (Mus musculus) and rat (Rattus norvegicus) cyclin E1 (outlined in red). C: Sequence comparison of the degron region in cyclin E1 (bottom) to the same region in cyclin E2 (top). Chou-Fasman analysis of the amino acid sequences suggests that the structures differ between cyclin E1 and cyclin E2. Additionally, the Cul3 degron in cyclin E1 is acidic, with a net charge of minus two (difference between the number of acidic residues and the number of basic residues). The same region in cyclin E2 is extremely basic with a net charge of plus four.

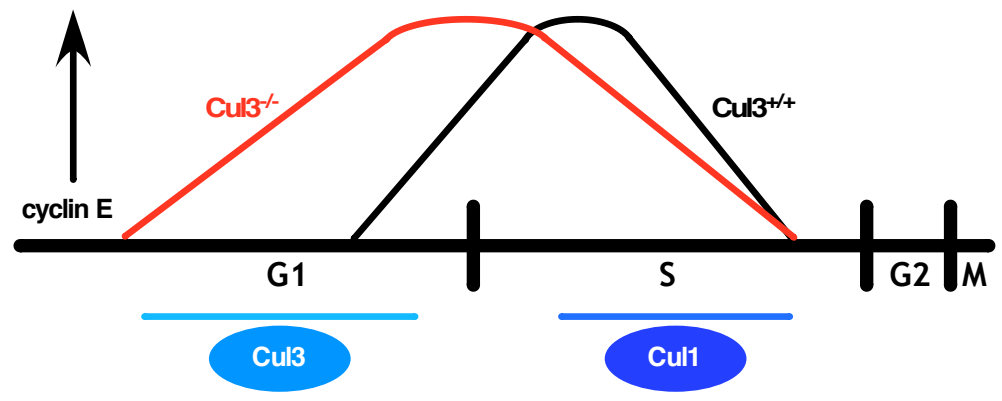

Figure 2.18: Cul3 degrades cyclin $E$ during $G_{1}$. Our data is consistent with a model where Cul3 is responsible for maintaining cyclin $E$ levels during $G_{1}$ in order to prevent early entrance into S-phase. Cul1 is known to degrade cyclin E after Sphase has begun, so therefore the two ligases work to degrade the cyclin at two different points during the cell cycle. 


\section{MATERIALS AND METHODS}

Cell culture and transfections: Cells (HEK 293, HeLa, and MEFs) were maintained in DMEM supplemented with ten percent fetal bovine serum and penicillin/streptomycin. HEK 293 and HeLa cells were split 1:20 for transfection in $6 \mathrm{~cm}$ dishes the night before transfection. Transfections were completed using calcium phosphate to precipitate the DNA onto the cells. For immunoprecipitations and expression level assays, cells were harvested 48 hours post transfection. For HeLa and 293 cells, between 1 and $10 \mu \mathrm{g}$ of plasmid DNA was transfected into each plate. For experiments using cycloheximide, drug was added 24 hours posttransfection at a final concentration of $50 \mu \mathrm{g}$ per milliliter. Cells were then harvested at the time points indicated. For experiments utilizing MG132, drug was added approximately 18 hours before harvest at a final concentration of $20 \mu \mathrm{M}$. All transfections were harvested using RIPA buffer and then sonicated before being used for immunoprecipitations or western blots. CRISPR knockout Cul3 293 cells were a gift from Curt Sigmund.

Plasmids: 3x-Flag-Cul3 was used for all Cul3-containing transfections. All Cul3

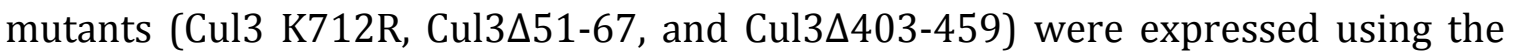
same p3x-Flag vector as wild-type Cul3. Cyclin E mutants were expressed using the CS2+ Myc-tagged expression vector, and CS2+ S-tagged and CS2+HA tagged vectors were utilized for expression of BTB proteins and the E2 enzyme UbE2E1.

Western blotting and immunoprecipitations: Western blots and immunoprecipitations were conducted as previously described (Wimuttisuk et al. 
2014). In short, a sonicated transfection lysate was added to the desired antibody in an Eppendorf tube and brought to a final volume of $500 \mu \mathrm{L}$. $40 \mu \mathrm{L}$ of IPA sepharose beads were then added to the mixture, and the IPs were placed on a rotator for two hours at room temperature before being rinsed with RIPA buffer, heated in SDSloading buffer, and run on an SDS-PAGE gel. The following antibodies were used for immunoprecipitations and/or western blotting: Monoclonal anti-FLAG (Sigma, F1804-50UG), monoclonal anti-Myc (9E10, Santa Cruz), polyclonal anti-c-Myc (A14) (Santa Cruz, discontinued), S-peptide monoclonal antibody (6.2) (Fisher, Cat\# MA1981), monoclonal anti-HA.11 (16B12) (ThermoFisher), polyclonal anti-HA (ThermoFisher, Product \# PA1-985), polyclonal anti- $\beta$ actin (ThermoFisher, Product \# PA1-183), polyclonal anti-cyclin E (Singer et al. 1999), polyclonal anti-Cul3 (Singer et al. 1999; McEvoy et al. 2007), and monoclonal anti-cyclin E (HE12, Santa Cruz biotechnology). Quantification of the western blots for all cycloheximide experiments was done using the FluorChem SP software (Alpha Innotech) and graphs were generated using Microsoft Excel.

Immunofluorescence: Hela cells were grown on coverslips, transfected, followed by incubation in four percent paraformaldehyde/PBS at room temperature for 10 minutes. Cells were then permeablized using a solution of one percent Triton X-100 with 2mM EGTA and 5mM PIPES, followed by incubation in methanol at -20 degrees for 10 minutes. Cells were then rinsed with PBS and stained overnight with a polyclonal Myc antibody (A14, Santa Cruz). The next day, cells were rinsed and stained with an AlexaFluor 488 conjugated secondary antibody (Abcam, ab150077) 
followed by DAPI, rinsed in methanol, and mounted on coverslips for viewing. Microscopy was conducted using a Zeiss M2 microscope and AxioVision software.

Flow cytometry: Proliferating cells were harvested when they were 70 percent confluent. Cells were resuspended in 70 percent ethanol in PBS and stored at 4 degrees until analysis. Prior to analysis, fixed cells were stained in a solution of propidium iodide in PBS with RNAse A at 37 degrees for a minimum of 30 minutes. Cells were then strained and analyzed on a BD Accuri C6 benchtop flow cytometer. Three proliferating samples of each genotype were analyzed and 20,000-50,000 cells were counted for each sample. Analysis shown in figure 2.10 was completed using FlowJo software (TreeStar). Cell cycle analysis of the samples in Figure 2.11 was completed by hand.

Construction of cyclin E mutants: The majority of the alanine scanning mutants were a kind gift from Jim Roberts at the Fred Hutchinson Cancer Center (Kelly et al. 1998). The point mutants were made using site-directed mutagenesis and all point mutants were confirmed by sequencing. Alanine scanning mutants that demonstrated a phenotype were also re-confirmed by sequencing. Truncations were cloned by using mutagenesis to generate a stop codon at the designated location in the cyclin E protein. Both deletions were made using site-directed mutagenesis and were also confirmed by sequencing. Forward primer sequences for mutagenesis are as follows: Cyclin E K80R (DKED point mutant) 5'TCCCCACACCTGACAGAGAAGATGATGACCG, Cyclin E 3 lysine (K118, 123, 125R) 5'AGAGGAAGTCTGGAGAATCATGTTAAACAGGGAAAGGACATACTTAAGGG, Cyclin E $\Delta$ 2-86 5'-GGACTTGAATTCCATGGTTTACCCAAACTCAA, Cyclin E KIDR point mutant 65 
(K48R) 5' -CGCCGTCCTGTCGATTCTGGCCATTTCTTCAT, Cyclin EA31-82 5'GCTCGCTCCAGGAAGGATGACCGGGTTTAC. Primer sequences for the cyclin E truncations are as follows: Cyclin E STOP 200 5'TCATCTTTATTTATTTGAGCCAAACTTGAGGAA, and STOP 300 5'TTTCCTTATGGTATATGAGCTGCTTCGGCCTGG. All reverse primers were reverse complements of the forward primers.

\section{REFERENCES}

Akli S, Zheng PJ, Multani AS, Wingate HF, Pathak S, Zhang N, Tucker SL, Chang S, Keyomarsi K. 2004. Tumor-specific low molecular weight forms of cyclin E induce genomic instability and resistance to p21, p27, and antiestrogens in breast cancer. Cancer research 64: 3198-3208.

Bagheri-Yarmand R, Biernacka A, Hunt KK, Keyomarsi K. 2010. Low molecular weight cyclin $\mathrm{E}$ overexpression shortens mitosis, leading to chromosome missegregation and centrosome amplification. Cancer research 70: 50745084.

Bhaskaran N, van Drogen F, Ng HF, Kumar R, Ekholm-Reed S, Peter M, Sangfelt O, Reed SI. 2013. Fbw7alpha and Fbw7gamma collaborate to shuttle cyclin E1 into the nucleolus for multiubiquitylation. Molecular and cellular biology 33: 85-97.

Boyden LM, Choi M, Choate KA, Nelson-Williams CJ, Farhi A, Toka HR, Tikhonova IR, Bjornson R, Gharavi AG, Goilav B et al. 2012. Mutations in kelch-like 3 and cullin 3 cause hypertension and electrolyte abnormalities. Nature Genetics: 111.

Chou PY, Fasman GD. 1975. The conformation of glucagon: predictions and consequences. Biochemistry 14: 2536-2541.

Clurman BE, Sheaff RJ, Thress K, Groudine M, Roberts JM. 1996. Turnover of cyclin E by the ubiquitin-proteasome pathway is regulated by cdk2 binding and cyclin phosphorylation. Genes Dev 10: 1979-1990.

Delk NA, Hunt KK, Keyomarsi K. 2009. Altered subcellular localization of tumorspecific cyclin $\mathrm{E}$ isoforms affects cyclin-dependent kinase 2 complex formation and proteasomal regulation. Cancer research 69: 2817-2825.

Duda DM, Borg LA, Scott DC, Hunt HW, Hammel M, Schulman BA. 2008. Structural insights into NEDD8 activation of cullin-RING ligases: conformational control of conjugation. Cell 134: 995-1006. 
Duong MT, Akli S, Wei C, Wingate HF, Liu W, Lu Y, Yi M, Mills GB, Hunt KK, Keyomarsi K. 2012. LMW-E/CDK2 deregulates acinar morphogenesis, induces tumorigenesis, and associates with the activated b-Raf-ERK1/2mTOR pathway in breast cancer patients. PLoS genetics 8: e1002538.

Geng Y, Yu Q, Sicinska E, Das M, Schneider JE, Bhattacharya S, Rideout WM, Bronson RT, Gardner H, Sicinski P. 2003. Cyclin E ablation in the mouse. Cell 114: 431443.

Grice GL, Nathan JA. 2016. The recognition of ubiquitinated proteins by the proteasome. Cellular and molecular life sciences : CMLS 73: 3497-3506.

Hao B, Oehlmann S, Sowa ME, Harper JW, Pavletich NP. 2007. Structure of a Fbw7Skp1-cyclin E complex: multisite-phosphorylated substrate recognition by SCF ubiquitin ligases. Molecular cell 26: 131-143.

Harwell RM, Porter DC, Danes C, Keyomarsi K. 2000. Processing of cyclin E differs between normal and tumor breast cells. Cancer research 60: 481-489.

Honda R, Lowe ED, Dubinina E, Skamnaki V, Cook A, Brown NR, Johnson LN. 2005. The structure of cyclin E1/CDK2: implications for CDK2 activation and CDK2independent roles. The EMBO journal 24: 452-463.

Ibeawuchi SR, Agbor LN, Quelle FW, Sigmund CD. 2015. Hypertension-causing Mutations in Cullin3 Protein Impair RhoA Protein Ubiquitination and Augment the Association with Substrate Adaptors. The Journal of biological chemistry 290: 19208-19217.

Jin J, Harper JW. 2002. RING finger specificity in SCF-driven protein destruction. Developmental cell 2: 685-687.

Kelly BL, Wolfe KG, Roberts JM. 1998. Identification of a substrate-targeting domain in cyclin E necessary for phosphorylation of the retinoblastoma protein. Proceedings of the National Academy of Sciences of the United States of America 95: 2535-2540.

Koepp DM, Schaefer LK, Ye X, Keyomarsi K, Chu C, Harper JW, Elledge SJ. 2001. Phosphorylation-dependent ubiquitination of cyclin E by the SCFFbw7 ubiquitin ligase. Science (New York, NY) 294: 173-177.

Koff A, Cross F, Fisher A, Schumacher J, Leguellec K, Philippe M, Roberts JM. 1991. Human cyclin E, a new cyclin that interacts with two members of the CDC2 gene family. Cell 66: 1217-1228.

Kwon JE, La M, Oh KH, Oh YM, Kim GR, Seol JH, Baek SH, Chiba T, Tanaka K, Bang OS et al. 2006. BTB domain-containing speckle-type POZ protein (SPOP) serves as an adaptor of Daxx for ubiquitination by Cul3-based ubiquitin ligase. The Journal of biological chemistry 281: 12664-12672.

Libertini SJ, Robinson BS, Dhillon NK, Glick D, George M, Dandekar S, Gregg JP, Sawai E, Mudryj M. 2005. Cyclin E both regulates and is regulated by calpain 2, a protease associated with metastatic breast cancer phenotype. Cancer research 65: 10700-10708.

Loeb KR, Kostner H, Firpo E, Norwood T, Tsuchiya KD, Clurman BE, Roberts JM. 2005. A mouse model for cyclin E-dependent genetic instability and tumorigenesis. Cancer Cell 8: 35-47. 
Lu A, Pfeffer SR. 2013. Golgi-associated RhoBTB3 targets cyclin E for ubiquitylation and promotes cell cycle progression. The Journal of cell biology 203: 233-250. McCormick JA, Yang CL, Zhang C, Davidge B, Blankenstein KI, Terker AS, Yarbrough B, Meermeier NP, Park HJ, McCully B et al. 2014. Hyperkalemic hypertensionassociated cullin 3 promotes WNK signaling by degrading KLHL3. The Journal of clinical investigation 124: 4723-4736.

McEvoy J, Kossatz U, Malek N, Singer J. 2007. Constitutive turnover of cylin E by Cul3 maintains quiescence. Molecular Cell Biology 27: 3651-3666.

Minella AC, Loeb KR, Knecht A, Welcker M, Varnum-Finney BJ, Bernstein ID, Roberts JM, Clurman BE. 2008. Cyclin E phosphorylation regulates cell proliferation in hematopoietic and epithelial lineages in vivo. Genes Dev 22: 1677-1689.

Perez-Neut M, Shum A, Cuevas BD, Miller R, Gentile S. 2015. Stimulation of hERG1 channel activity promotes a calcium-dependent degradation of cyclin E2, but not cyclin E1, in breast cancer cells. Oncotarget 6: 1631-1639.

Petroski MD, Deshaies RJ. 2005. Function and regulation of cullin-RING ubiquitin ligases. Nature reviews Molecular cell biology 6: 9-20.

Plafker KS, Singer JD, Plafker SM. 2009. The ubiquitin conjugating enzyme, UbcM2, engages in novel interactions with components of cullin-3 based E3 ligases. Biochemistry 48: 3527-3537.

Porter DC, Keyomarsi K. 2000. Novel splice variants of cyclin E with altered substrate specificity. Nucleic acids research 28: E101.

Porter DC, Zhang N, Danes C, McGahren MJ, Harwell RM, Faruki S, Keyomarsi K. 2001. Tumor-specific proteolytic processing of cyclin $\mathrm{E}$ generates hyperactive lower-molecular-weight forms. Molecular and cellular biology 21: 6254-6269.

Rath SL, Senapati S. 2014. Why are the truncated cyclin Es more effective CDK2 activators than the full-length isoforms? Biochemistry 53: 4612-4624.

Richardson HE, O'Keefe LV, Reed SI, Saint R. 1993. A Drosophila G1-specific cyclin E homolog exhibits different modes of expression during embryogenesis. Development 119: 673-690.

Rogers S, Wells R, Rechsteiner M. 1986. Amino acid sequences common to rapidly degraded proteins: the PEST hypothesis. Science 234: 364-368.

Rogers SW, Rechsteiner MC. 1986. Microinjection studies on selective protein degradation: relationships between stability, structure, and location. Biomedica biochimica acta 45: 1611-1618.

Said TK, Medina D. 1995. Cell cyclins and cyclin-dependent kinase activities in mouse mammary tumor development. Carcinogenesis 16: 823-830.

Scuderi R, Palucka KA, Pokrovskaja K, Bjorkholm M, Wiman KG, Pisa P. 1996. Cyclin $\mathrm{E}$ overexpression in relapsed adult acute lymphoblastic leukemias of B-cell lineage. Blood 87: 3360-3367.

Sherr CJ, Roberts JM. 1999. CDK inhibitors: positive and negative regulators of G1phase progression. Genes Dev 13: 1501-1512.

Singer JD, Gurian-West M, Clurman B, Roberts JM. 1999. Cullin-3 targets cyclin E for ubiquitination and controls S phase in mammalian cells. Genes Dev 13: 23752387. 
Strohmaier H, Spruck CH, Kaiser P, Won KA, Sangfelt O, Reed SI. 2001. Human F-box protein hCdc4 targets cyclin $\mathrm{E}$ for proteolysis and is mutated in a breast cancer cell line. Nature 413: 316-322.

Tyers M, Jorgensen P. 2000. Proteolysis and the cell cycle: with this RING I do thee destroy. Current opinion in genetics \& development 10: 54-64.

Wang XD, Rosales JL, Magliocco A, Gnanakumar R, Lee KY. 2003. Cyclin E in breast tumors is cleaved into its low molecular weight forms by calpain. Oncogene 22: 769-774.

Welcker M, Singer J, Loeb KR, Grim J, Bloecher A, Gurien-West M, Clurman BE, Roberts JM. 2003. Multisite phosphorylation by Cdk2 and GSK3 controls cyclin E degradation. Molecular cell 12: 381-392.

Wimuttisuk W, Singer JD. 2007. The Cullin3 ubiquitin ligase functions as a Nedd8bound heterodimer. Molecular biology of the cell 18: 899-909.

Wimuttisuk W, West M, Davidge B, Yu K, Salomon A, Singer JD. 2014. Novel Cul3 binding proteins function to remodel E3 ligase complexes. BMC cell biology 15: 28.

Wingate H, Zhang N, McGarhen MJ, Bedrosian I, Harper JW, Keyomarsi K. 2005. The tumor-specific hyperactive forms of cyclin $\mathrm{E}$ are resistant to inhibition by p21 and p27. The Journal of biological chemistry 280: 15148-15157.

Zhang P, Gao K, Tang Y, Jin X, An J, Yu H, Wang H, Zhang Y, Wang D, Huang $\mathrm{H}$ et al. 2014. Destruction of DDIT3/CHOP protein by wild-type SPOP but not prostate cancer-associated mutants. Human mutation.

Zheng N, Schulman BA, Song L, Miller JJ, Jeffrey PD, Wang P, Chu C, Koepp DM, Elledge SJ, Pagano M et al. 2002. Structure of the Cul1-Rbx1-Skp1-F boxSkp2 SCF ubiquitin ligase complex. Nature 416: 703-709. 
CHAPTER 3: Identifying Possible Roles of Cul3 and Klhl3 in the Kidney 


\section{BACKGROUND: The role of Cul3 and Klhl3 in FHHt}

Mutations in the BTB-Kelch protein Klhl3 as well as Cul3 have recently been shown to cause an inherited form of hypertension called Familial Hyperkalemic Hypertension (FHHt) (Boyden et al. 2012; Louis-Dit-Picard et al. 2012). This form of hypertension results primarily from the misregulation of NCC sodium chloride cotransporter located on the apical membrane of the distal convoluted tubule of the nephron (O'Shaughnessy 2015). Klhl3 can affect NCC, resulting in disease, via degradation of the WNK4 kinase, which is responsible for controlling NCC through a phosphorylation pathway involving the SPAK and OSR1 kinases (summarized in Figure 3.1) (McCormick and Ellison 2011; Shibata et al. 2013). Mutations in Klhl3 that alter its ability to bind and ubiquitinate WNK4 result in changes in NCC phosphorylation status, which leads to the disease phenotype (Ohta et al. 2013; Shibata et al. 2013; Wakabayashi et al. 2013). Interestingly, the FHHt-causing mutations in Cul3 and Klhl3 are associated with a more severe disease phenotype than the previously known FHHt-causing mutations in WNK4 and the related kinase WNK1 (Boyden et al. 2012) . This information suggests that Klhl3 and Cul3 may have additional unknown roles in this pathway. In order to elucidate Cul3's role in FHHt, two approaches, one focused on Cul3 and one focused on Klhl3, were taken: First, the structure and function of the hypertension-associated Cul3 mutation, Cul3 $4403-459$, was studied, and secondly, a screen was performed in order to identify potential binding partners of Klhl3 in vivo. 


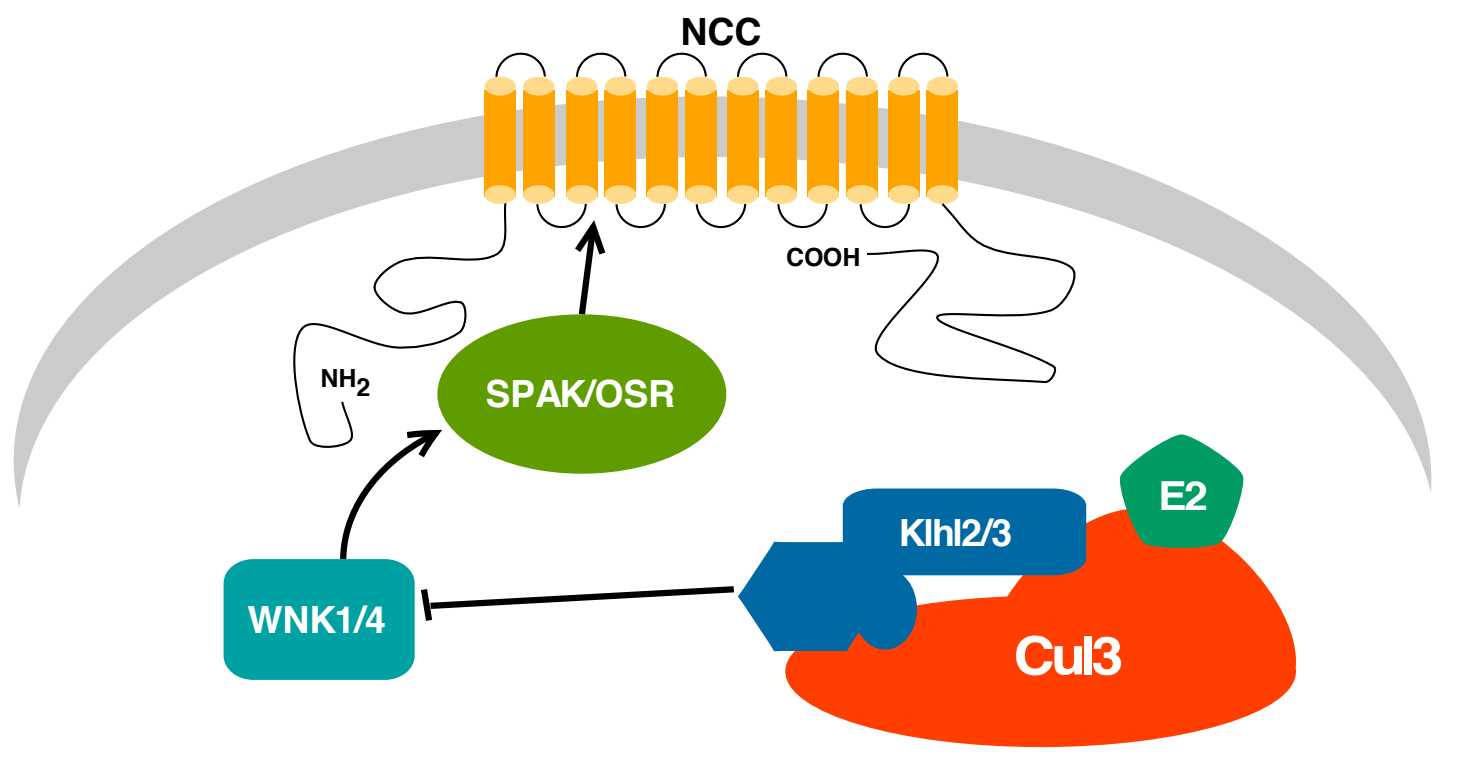

Figure 3.1: NCC regulation. NCC is regulated via phosphorylation, which requires multiple kinases such as SPAK, OSR1, and the WNK kinases. There are several possible points at which Cul3 can regulate this pathway: The Cul3 ${ }^{\mathrm{Klhl} 3} \mathrm{E} 3$ ligase has been shown to ubiquitinate WNK4, but other possible roles of Cul3 in this process remain unknown.

As previous work from our lab has demonstrated that Cul3 is an essential gene as its loss results in embryonic lethality in mice, we hypothesized that the newly discovered human Cul3 mutation is a functional ubiquitin ligase capable of ubiquitinating substrates and therefore the FHHt-associated Cul3 mutant is not a loss of function mutant (Singer et al. 1999). We sought to determine the biochemical properties of the human Cul3 mutations as well as the role of the Cul3 E3 ligase in the regulation of blood pressure (McCormick et al. 2014). This work, which was published in 2014, demonstrated that the hypertension-causing Cul3 mutant, Cul3 $\Delta 403-459$, is a gain of function mutant that has an increased ability to 
interact with the substrate adaptor Klhl3 as well as an increased ability to ubiquitinate substrates, including its substrate adaptor Klhl3 (McCormick et al. 2014). A kidney-specific deletion of the Cul3 gene in the DCT of mice revealed that in contrast to the human mutation, loss of Cul3 decreases blood pressure (McCormick et al. 2014). The kidneys also showed a marked increase in levels of WNK4, a SPAK regulating kinase that ultimately controls NCC function, which is consistent with the accepted idea that WNK4 is a substrate of Cul3 and Klhl3 (McCormick et al. 2014).

One interesting revelation from this study was the change in subcellular localization of the hypertension-causing Cul3 mutant (Figure 3.2). The localization of wild-type Cul3 is predominantly nuclear, but the mutant Cul3 $\Delta 403-459$ is mostly cytoplasmic. Normally, Cul3 is recruited to different locations in the cell by BTB proteins (Mathew et al. 2012), which is demonstrated here by the shift of wild-type Cul3 from the nucleus to the cytoplasm in the presence of Klhl3 (Figure 3.2 bottom row) (McCormick et al. 2014). This change in localization of the human Cul3 mutation indicates that the mutant Cul3 may have an altered ability to degrade substrates as a result of its improper localization, resulting in changes to ubiquitination that are substrate-dependent. Our lab has shown that Cul3 $\Delta 403-459$ can ubiquitinate cyclin E and Klhl3 (McCormick et al. 2014), but others have suggested that Cul3 $\Delta 403-459$ actually has an impaired ability to degrade the Cul3 substrate RhoA (Ibeawuchi et al. 2015). Future work investigating how Cul3 $\Delta 403$ 459 impacts different substrates will be necessary in order to better determine the 
differential regulation of Cul3 substrates that might occur in individuals who carry

this mutation.

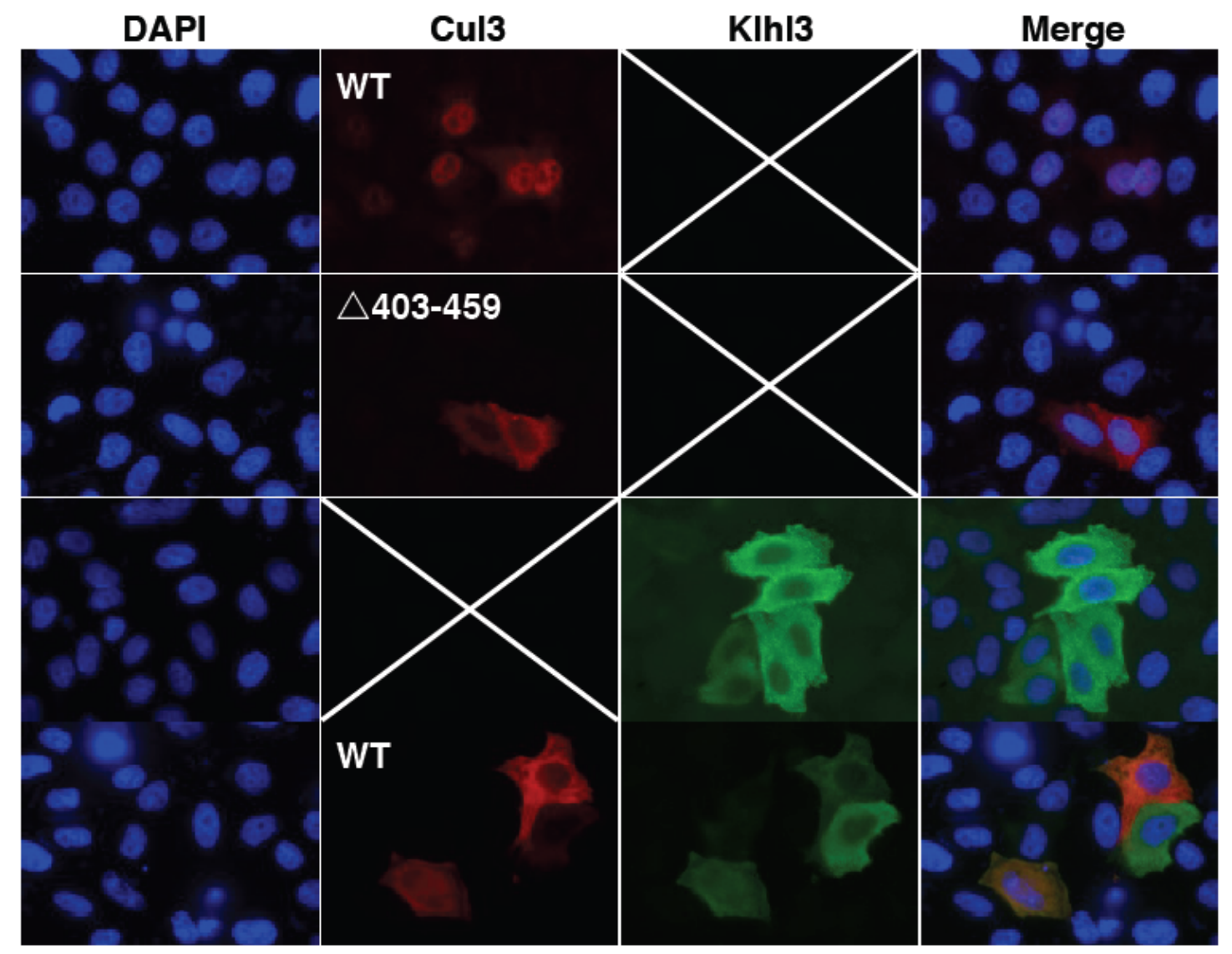

Figure 3.2: The human Cul3 mutant localizes to the cytoplasm. HeLa cells were transfected with wild-type (WT) Flag-Cul3, Flag-Cul3 $\Delta 403-459$, Myc-Klhl3, or both Myc-Klhl3 and Flag-Cul3 and stained for fluorescent microscopy. Flag or Myc antibodies were used to detect the transfected protein. WT Cul3 is predominantly nuclear (top row) whereas the mutant is mainly localized to the cytoplasm (second row). Myc-Klhl3 is a cytoplasmic protein (third row) and is able to recruit WT FlagCul3 to the cytoplasm (bottom row). The same pattern can be observed in mDCT kidney cells (McCormick et al. 2014). The experiment shown here has been published (McCormick et al. 2014). 


\section{RESULTS:}

\section{Identifying proteins that interact with Klhl3}

As mutations in the BTB-Kelch protein and putative Cul3 substrate adaptor, Klhl3, have also been shown to cause FHHt (Boyden et al. 2012; Louis-Dit-Picard et al. 2012), it is essential to gain a better understanding of Klhl3 and its role in the cell. One way to do this is to identify potential Klhl3 interacting partners and substrates in the cell. Several yeast two-hybrid screens were performed using full length Klhl3 (Figure 3.3). Klhl3 was cloned into a yeast expression vector and transformed into yeast. The Klhl3-containing colonies were then screened against a cDNA library to identify interacting proteins. Yeast two-hybrid screens are valuable for identifying protein-protein interactions, as they provide an inexpensive in vivo system that allows millions of proteins to be screened for interactions with a single 'bait' protein of interest. Klhl3 was utilized as 'bait' in the appropriate two-hybrid system to identify the possible interacting proteins listed below in Table 3.1.

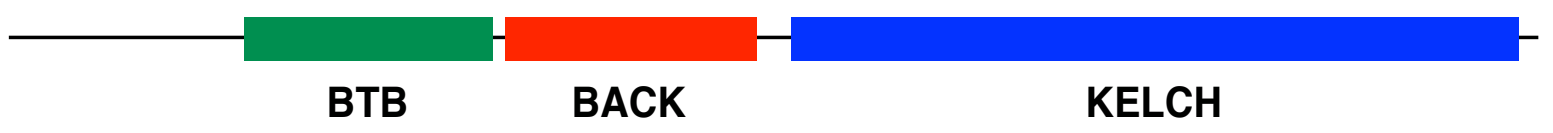

Figure 3.3: Klhl3 structure. Klhl3 is composed of an N-terminal BTB domain followed by a BACK domain and C-terminal Kelch domain. The BTB domain is responsible for binding Cul3 while the Kelch domain interacts with substrates. The BACK domain connects the BTB and Kelch domains. 
Table 3.1: Potential binding partners of Klhl3

\begin{tabular}{llll} 
Bait: & Library Used: & $\begin{array}{l}\text { Number of Clones } \\
\text { screened: }\end{array}$ & $\begin{array}{l}\text { ID of interacting } \\
\text { partners: }\end{array}$ \\
\hline KLHL3 & Human Testis & $1.2 \times 10^{6}$ & $\begin{array}{l}\text { Dynamitin }(\mathrm{p} 50) \\
\text { PHLDB3 } \\
\text { Gef10 }\end{array}$ \\
& & & PIASx \\
& & KLHL26 \\
& & & Dynamitin (p50) \\
KLHL3 & Human Fetal & \multirow{2}{*}{$3.0 \times 10^{6}$} & \\
& Liver & & Dynamitin (p50) \\
KLHL3 & Human Kidney & \multirow{2}{*}{$1.7 \times 10^{6}$} & KLHL3 \\
& & & TGF $\beta$ receptor \\
& & & TGF $\beta$ receptor 2 \\
& & & RANBP2 \\
& & & RANBP9 \\
& & & MUC20 \\
& & ATP1B1 & Foxp1
\end{tabular}

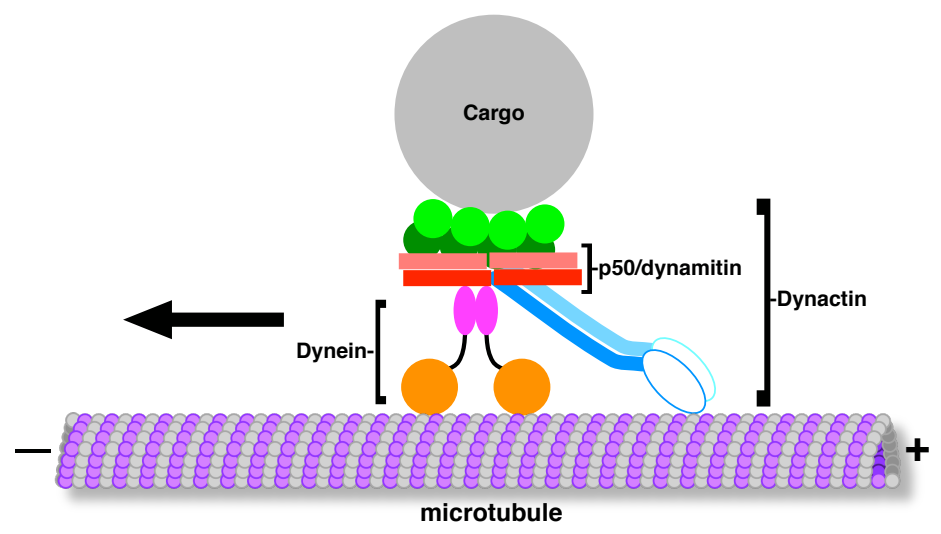

Figure 3.4: $\quad$ P50/dynamitin is a subunit of the dynactin complex. P50 interacts with the dynein motor. P50 forms the scaffold, which connects the cargo-binding arm (Arp1, green) to the microtubule-binding (p150/Glued, blue) domain. This complex is necessary for transport of endosomes within the cell. 


\section{Dynamitin/p50 binds Klhl3}

One protein of interest that was identified in all three screens was p50/dynamitin. Dynamitin/p50 is a subunit of the dynactin complex, which is required for retrograde transport of endocytic vesicles (Figure 3.4). The p50/dynamitin subunit is of interest as a possible Klhl3 binding partner as p50 overexpression is known to result in trafficking errors and breakdown of the dynein-dynactin complex (Burkhardt et al. 1997), making p50 a good candidate for regulation by the ubiquitin-proteasome system.

P50 was cloned by PCR into a CS+ vector that contained either HA and S epitope tag. These vectors allow the protein of interest to be expressed in mammalian cells and carry an epitope tag for easy detection. Immunofluorescent microscopy reveals that transfected Klhl3 and p50 co-localize in the cytoplasm, which means there is a possibility that a biologically relevant interaction might occur between these two proteins (Figure 3.5). Co-transfection of Klhl3 and p50 followed by immunoprecipitation revealed the ability of the two proteins to interact in mammalian cells (Figure 3.6, lane 1 compared to lane 3). In order to further determine the significance of the interaction between p50 and Klhl3, information was sought in the literature regarding the staining pattern of the endogenous proteins. Endogenous Klhl3 is known to stain in a punctate pattern in 293 cells (Louis-Dit-Picard et al. 2012), which can be characteristic of actin-associated proteins. Endogenous p50 is also known to stain in a similar cytoplasmic pattern (Burkhardt et al. 1997). Taken together, this information suggests that p50 and 
Klhl3 interact in vivo and it is possible that Klhl3 is normally found associated with the dynactin complex in the cytoplasm.

To further elucidate the interaction that occurs between p50 and Klhl3, a Klhl3 mutant which lacks the BTB (Cul3 binding, refer to figure 3.3) domain was created. This construct was utilized to determine if the kelch domain of Klhl3, which is involved in substrate recognition for the Cul3 ubiquitin ligase complex, is the portion of Klhl3 that is responsible for interacting with p50. This construct also contains the BACK domain, which connects the BTB domain to the Kelch domain. Either wild-type Klhl3 or Klhl3 $\Delta$ BTB was co-expressed with p50 (Figure 3.6). P50 binds Klhl3 $\triangle \mathrm{BTB}$ and is in fact stabilized in its presence (Figure 3.6, lane 4 compared to lane 2), suggesting that p50 binds to the substrate recognition region (kelch domain) and normally is degraded in a Cul3-dependent fashion. This result indicates that $\mathrm{Klhl} 3 \triangle \mathrm{BTB}$ is sequestering p50 and protecting it from Cul3-mediated degradation, as the kelch domain of Klhl3 retains its high affinity for p50, but without the BTB domain is unable to associate with the Cul3 complex so p50 is not degraded. This result supports the hypothesis that p50 is a substrate of Klhl3 and Cul3. 


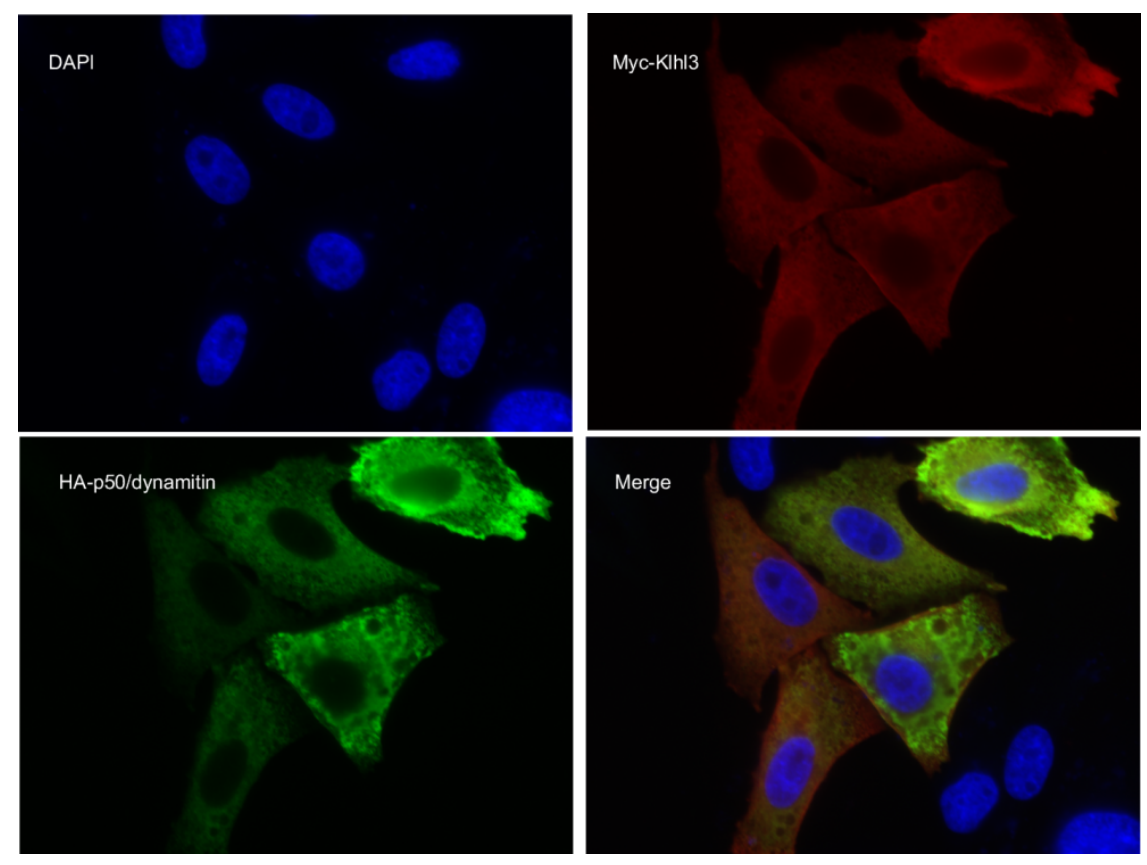

Figure 3.5: $P 50$ and Klhl3 co-localize in the cytoplasm. Ha-p50 and Myc-Klhl3 were transfected into HeLa cells and stained using antibodies to HA and/or Myc, and viewed using immunofluorescent microscopy. The localization of p50 is shown in green, Klhl3 in red, and the nucleus (DAPI) in blue. 


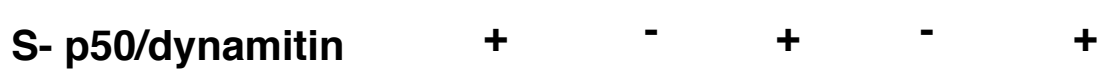

$\begin{array}{llllll}\text { Myc- KIhI3 } & - & \text { WT } & \text { WT } & \Delta B T B & \Delta B T B\end{array}$

IP: S-tag

WB: Myc

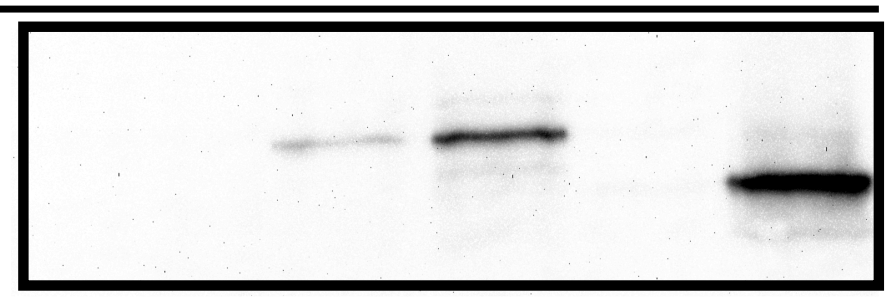

WB: Myc

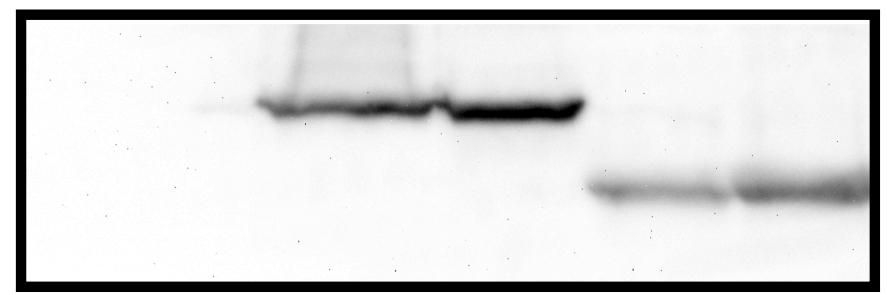

WB: S-tag

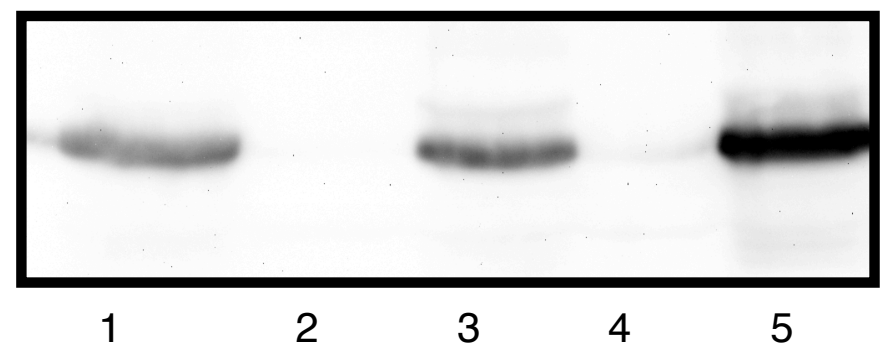

Figure 3.6: $P 50$ interacts with the substrate recognition domain of Klhl3. Upper blot; immunoprecipitation results showing binding between S-tagged p50 and either wild-type Klhl3 (lanes 2 and 3), or Klhl3 lacking its BTB domain (lanes 4 and 5). The middle and lower blots show protein expression in the cell extracts.

\section{P50 is stabilized in the presence of an FHHt-associated Klhl3 mutant}

Several mutations in Klhl3 have been shown to cause FHHt in humans (Boyden et al. 2012; Louis-Dit-Picard et al. 2012). A dominant mutation that results in hypertension is Klhl3R528H, a point mutation in the kelch domain of Klhl3 (Boyden et al. 2012). In order to determine if this mutant affects p50, the two proteins were co-transfected in HEK293 cells (Figure 3.7 lane 2). Our preliminary results show that p50 appears to be stabilized by Klhl3R528H but not wild-type 
Klhl3 (Figure 3.7 lane 2 compared to lanes 1 and 4). The increased stability is comparable to the stability that results when p50 is expressed with Klhl3 that cannot bind Cul3 (lacks BTB domain, figure 3.7 lane 3). P50 is still able to bind Klhl3R528H (Figure 3.7 bottom row lane 2). Taken together, these results suggest that Klhl3-mediated degradation might be impaired in the presence of Klhl3R528H.

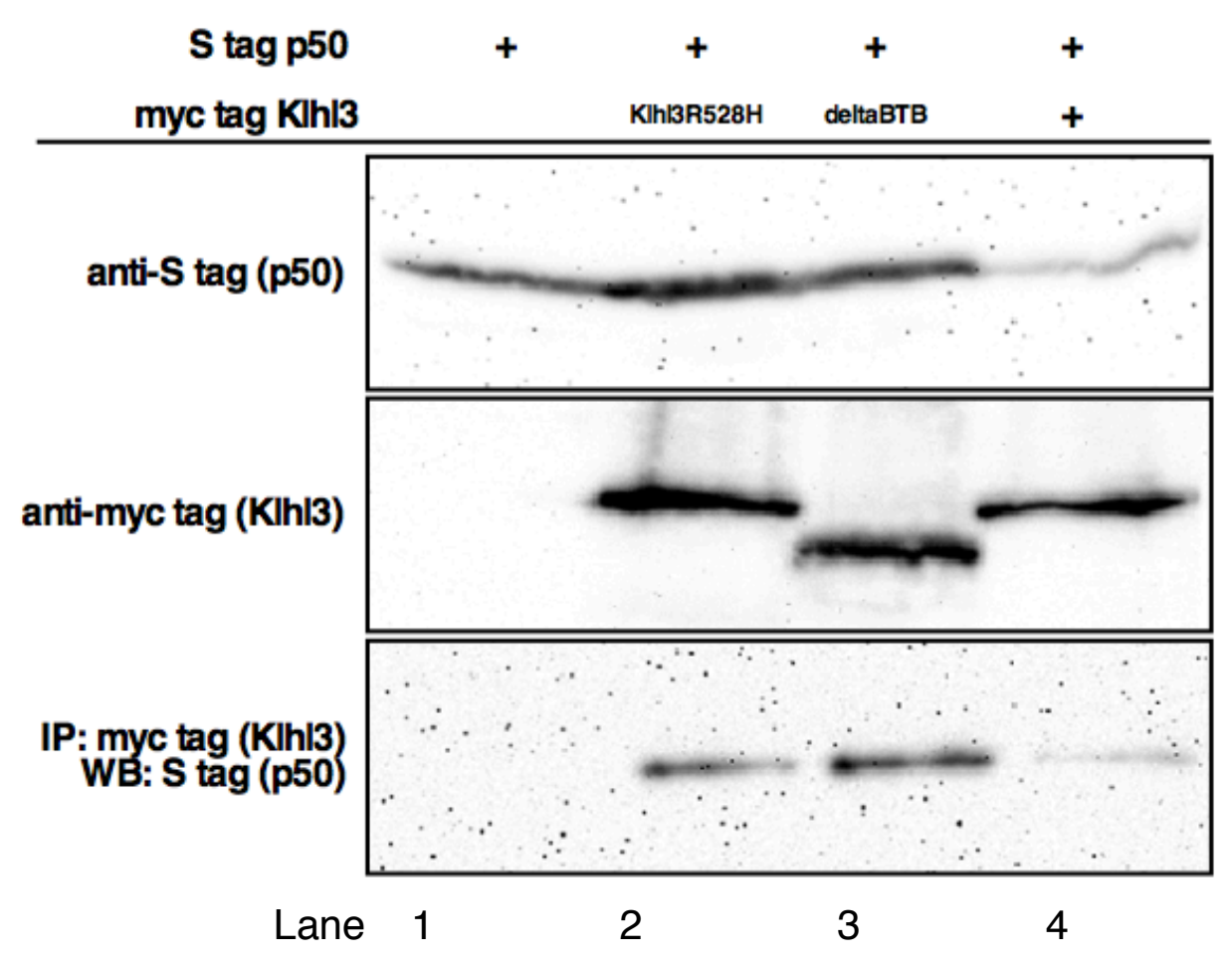

Figure 3.7: A human Klhl3 mutant results in p50 stability. S-tagged p50 was cotransfected with a human Klhl3 mutation, Klhl3R528H (lane 2), Klhl3 lacking its BTB domain (cannot bind Cul3, lane 3), or wild-type Klhl3 (Lane 4). P50 expression levels in the presence of each Klhl3 mutant are shown (top panel). The middle blot shows Klhl3 expression and the lower blot shows immunoprecipitation results. This is a preliminary result as it has not yet been repeated. 


\section{P50 binds Cul3}

As previously demonstrated in chapter two, the Cul3 substrate cyclin E is able to bind Cul3 in addition to a BTB substrate adaptor. In order to determine if p50 also possesses this capability, it was checked for binding with a set of Cul3 mutants. It was concluded that, similar to cyclin E, p50 also has the ability to bind wild-type Cul3 in addition to several Cul3 mutants (Figure 3.8, lanes 2-6). In addition to WT Cul3, p50 also binds Cul3 $\Delta 51-67$, a mutant that does not bind BTB proteins as well as Cul3K712R, an inactive Cul3 that is not modified by Nedd8 (Figure 3.8, lanes 3 and 5). These data suggest that p50 may be able to interact with Cul3 independently of its interaction with Klhl3 and this interaction is not dependent on neddylation of Cul3. P50 was also able to bind the Cul3 mutant that is associated with hypertension, Cul3 $\Delta 403-459$, indicating that the region of Cul3 that is deleted in this mutant is not involved in the interaction with p50 (Figure 3.8, lane 4), as well as a double mutant which contains both the hypertension mutation and the BTB binding mutation (Figure 3.8, lane 6). 


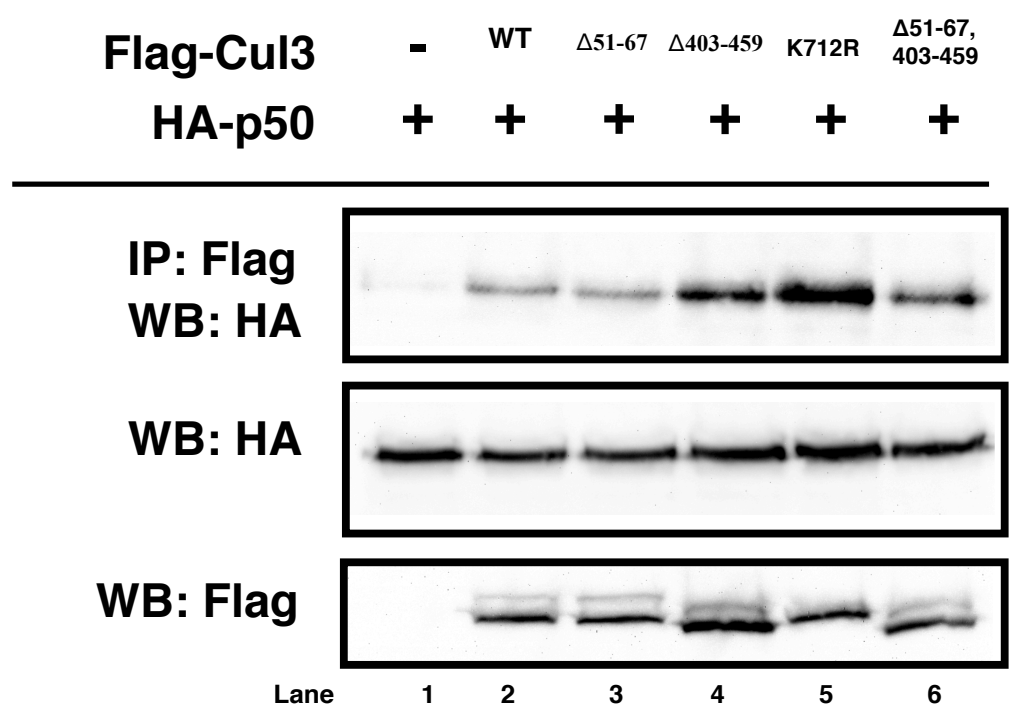

Figure 3.8: $\quad$ P50 binds Cul3 and Cul3 mutants. Upper blot; S-p50 was transfected with several Cul3 mutants. Immunoprecipitation results are shown. The lower lower blots show expression levels of the transfected proteins in the cell lysates.

\section{Cul3 and Klhl3 ubiquitinate p50/dynamitin in vitro}

The data presented above suggest that p50/dynamitin can serve as a substrate of Cul3 and Klhl3 in vitro (Figures 3.5 and 3.9). In order to further investigate this hypothesis and determine if p50 is a substrate of the ubiquitinproteasome pathway, S-tagged p50 was co-transfected with Cul3 and Klhl3 in the presence or absence of the proteasome inhibitor MG132. P50 levels are destabilized when Cul3 and Klhl3 are present, but its levels are restored by MG132 (Figure 3.9 lane 3), demonstrating that the observed reduction in p50 stability in the presence of Klhl3 or Cul3 may be a result of proteasomal degradation. Additionally, cotransfection of Ubr7, a mutant ubiquitin where all lysines have been mutated to arginine rendering it unable to form degradative ubiquitin chains, also increases 
steady-state levels of p50 (Figure 3.9 lanes 4 and 5). Ubiquitin laddering of the p50 protein can also be observed, which suggests that is may be a substrate of ubiquitination (Figure 3.9 lanes 4 and 5). Taken together, these data support the hypothesis that $\mathrm{p} 50$ is ubiquitinated by Cul3 and Klhl3 in vitro.

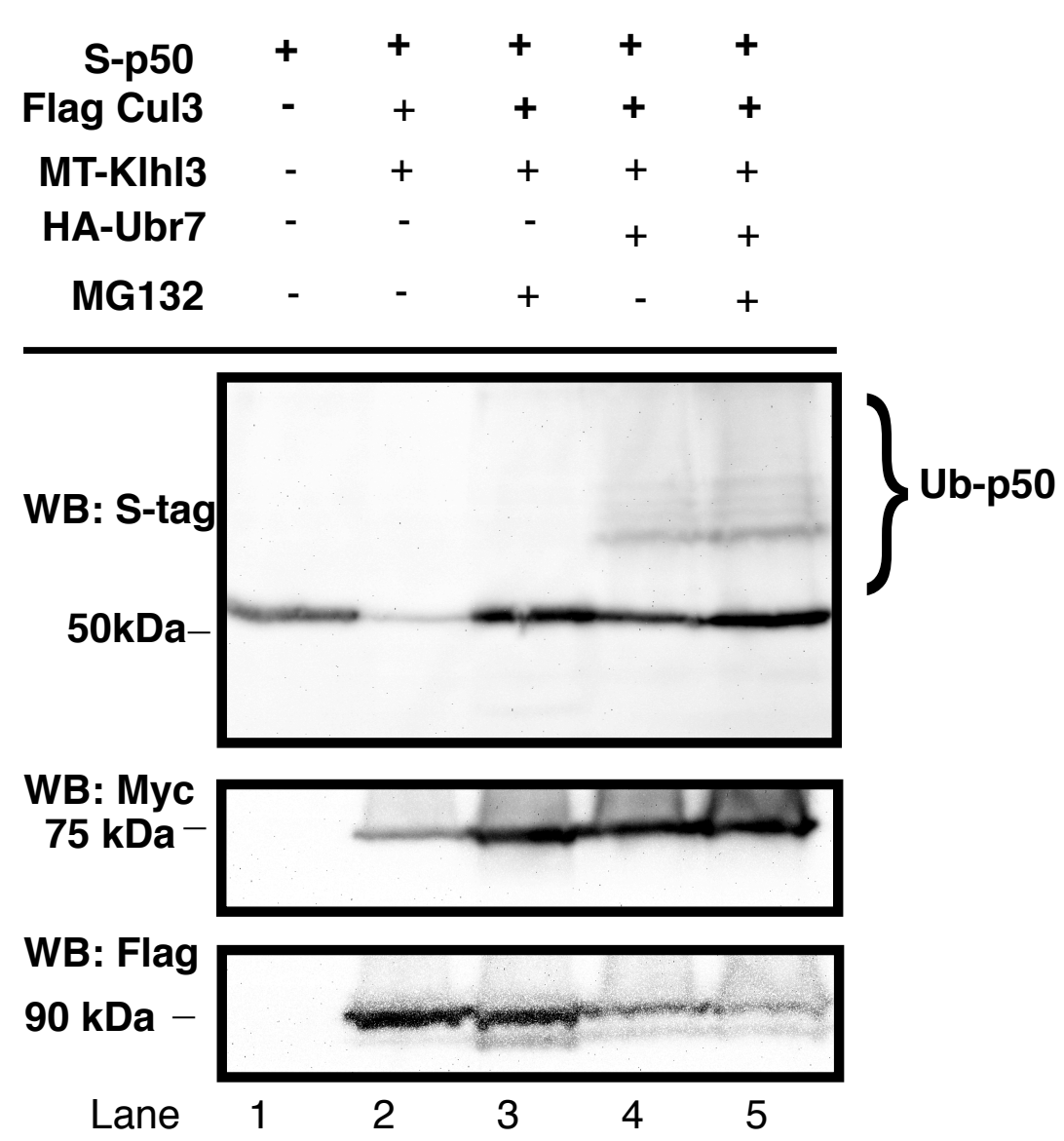

Figure 3.9: $\quad$ P50 is ubiquitinated in the presence of Cul3 and Klhl3. S-tagged Dynamitin/p50 expression levels are shown either alone (Top panel, lane 1) or in the presence of Cul3 and Klhl3 (lanes 2 through 5), with or without the ubiquitin mutant Ubr7 (lanes 4 and 5) or the proteasome inhibitor MG132 (lanes 3 and 5). 


\section{P50 levels appear to remain unchanged in vivo}

In order to determine if p50 is targeted for degradation by Cul3 in vivo, levels of p50 were compared in Cul3 WT and Cul3 KO 293 cells (Ibeawuchi et al. 2015). P50 levels appear evenly expressed in the wild-type and Cul3 KO cells (Figure 3.10). This result indicates that if p50 is a substrate of Cul3, ubiquitination may serve a purpose besides degradation, or ubiquitination results in degradation but only of a small pool of p50, which could occur either in a cell cycle or localization-dependent manner. Based on the convincing data demonstrating the stabilization of p50 by transfected ubiquitin mutants or the addition of MG132 (Figure 3.9), the latter possibility seems more likely, as p50 would show no change in stability if ubiquitination by Cul3 were non-degradative.

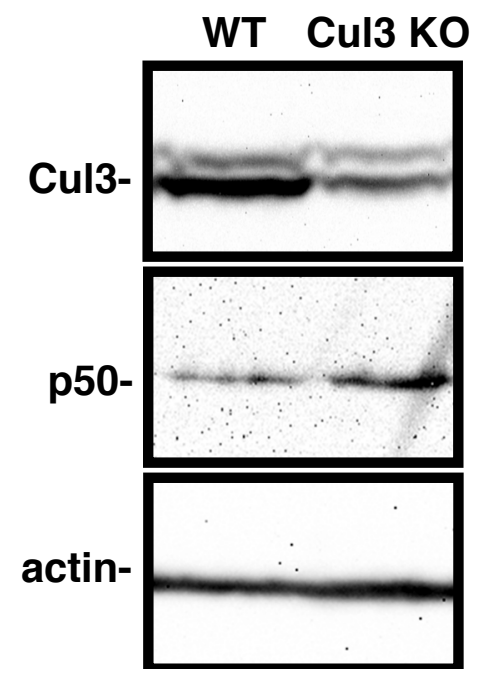

Figure 3.10: Levels of endogenous $p 50$ are unaffected by loss of Cul3. Levels of endogenous p50 (middle panel) in wild-type and Cul3 KO 293 cells were determined via Western blotting. Levels of actin in the same cells (bottom panel) are shown for comparison. 


\section{Transfected p50 is destabilized by Cul3}

To further support the hypothesis that p50 is a Cul3 substrate, it was transfected into the Cul3 WT and Cul3 KO 293 cells to check its stability in the presence and absence of Cul3 (refer to the assay in Chapter 2 Figure 2.4). Like cyclin E, a known Cul3 substrate, p50 appears more stable in the KO cells that it does in the wild-type (Figure 3.11, top panel). This result further supports a role of Cul3 in p50 ubiquitination.

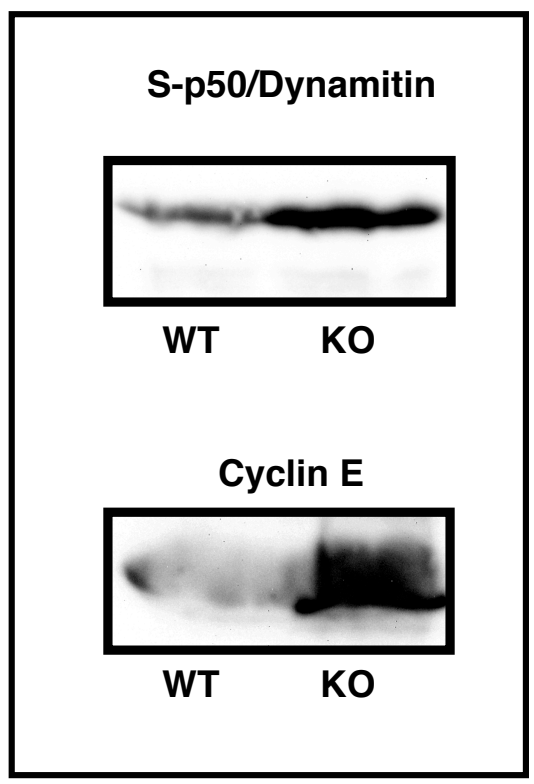

Figure 3.11: $\quad$ Transfected $p 50$ is more stable in Cul3 KO cells. Western blot showing levels of transfected S-p50 in Cul3 KO 293 cells (top panel, right lane) in comparison to WT cells (top panel, left lane). The Cul3 substrate cyclin E is shown as a control (bottom panel).

\section{DISCUSSION:}

Mutations in Klhl3, a BTB protein and putative substrate adaptor for Cul3, have been shown to cause the inherited form of hypertension FHHt (Boyden et al. 2012; Louis-Dit-Picard et al. 2012). Others have shown that Klhl3 plays a role in the 
ubiquitination of the kinases WNK1 and WNK4, which are responsible for phosphorylating the SPAK kinase, which in turn regulates NCC (Yang et al. 2003; Ohta et al. 2013; Shibata et al. 2013; Wakabayashi et al. 2013). In order to determine other cellular processes that Klhl3 might regulate, a yeast two-hybrid screen was performed to identify potential binding partners of Klhl3 (Table 3.1). One protein that this screen identified is p50/dynamitin, a subunit of the dynactin complex, which is involved in vesicle trafficking along the cytoskeleton (Table 3.1 and Figure 3.4). The p50/dynamitin subunit of the dyanctin complex plays a role in dynein-dependent trafficking within the cell. The work presented here demonstrates that p50/dynamitin binds to the substrate recognition (Kelch) domain of Klhl3 and also to Cul3, and can be ubiquitinated in a Cul3 or Klhl3depenent manner. Taken together, these results suggest that p50 is a likely substrate of the Cul3 ${ }^{\mathrm{KIhl} 3} \mathrm{E} 3$ ubiquitin ligase in cells (Figures 3.6, 3.7, 3.8, and 3.9). These findings suggest the possibility that Klhl3 might function as a regulator of p50 in cells, thereby suggesting a possible role for Klhl3 in endosomal trafficking.

\section{FUTURE DIRECTIONS:}

Taken together, these results suggest a role for $\mathrm{p} 50 /$ dynamitin as a substrate of Cul3 and Klhl3 in vitro. However, it remains unclear if p50 is ubiquitinated in a Cul3-dependent manner in living cells and organisms. Future experimentation will be necessary in order to determine the role Klhl3 plays in cells and to confirm the status of p50 as a Cul3 substrate and also show what affects p50 degradation may be having in cells. An experiment that would be illuminating would be to knock 
down Klhl3 using siRNA in a cell line where Klhl3 is known to be expressed and measuring if there are any effects on endosomal trafficking and endosome maturation in the Klhl3 knock-down cells. Antibodies to early endosome markers such as EEA1 and late endosome markers such as LAMP-1 would be useful for this experiment (Franken et al. 2013). In order to gather the most convincing results, it is important that Klhl3 is knocked-down for this experiment, as Cul3 has already been shown to affect vesicle trafficking via a different mechanism(Hubner and Peter 2012; Huotari et al. 2012; Gschweitl et al. 2016). An effective siRNA against Klhl3 has been developed for use in this experiment (Figure 3.12)

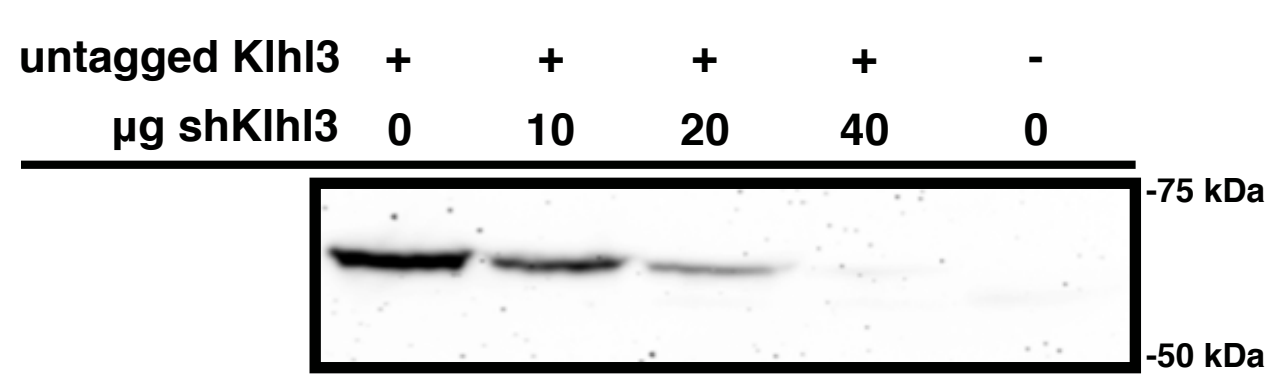

Figure 3.12: An siRNA targeting Klhl3 effectively decreases the expression of transfected Klhl3 protein. Different amounts of an siRNA targeting Klhl3 were transfected into 293 cells with Klhl3. This siRNA can be used in future experiments requiring the knockdown of Klhl3.

\section{The possibility of aquaporin regulation by Cul3}

One notable anomaly in adult mice that have had Cul3 deleted from their kidneys is depletion of the AQP2 aquaporin protein (McCormick et al. 2014). AQP2, a member of the aquaporin family of water channels, is located on the apical surface of the renal collecting duct, where it functions in the reabsorption of water, helping to maintain blood pressure and ion homeostasis (Takata et al. 2008). Expression of 
AQP2 at the apical membrane is highly regulated (Takata et al. 2008). AQP2 is stored in vesicles and is transported to the membrane in a dynein-mediated manner, which is contrary to the usual direction of dynein-mediated transport as a result of microtubules that are able to nucleate near the membrane in this instance (Marples et al. 1998). Translocation of AQP2 to the apical membrane occurs when intracellular cAMP levels are increased upon stimulation by the hormone vasopressin (Klussmann et al. 2001; Olesen and Fenton 2017). In order for translocation to occur, AQP2 must have been previously phosphorylated by PKA (Nedvetsky et al. 2009). There are several possible ways in which Cul3 might be involved in AQP2 regulation: 1) AQP2 could be a substrate of Cul3, 2 and 3) The Cul3 substrates Nrf2 or RhoA, which are believed to regulate AQP2 might be involved, and 4) Cul3 might regulate AQP2 via trafficking. These four possibilities as well as the literature supporting them will be discussed below.

When $\mathrm{AQP} 2$ is no longer needed at the membrane, it undergoes clatharinmediated endocytosis, which is also dynein-dependent (AQP2 recycling is summarized in Figure 3.13) (Takata et al. 2008; Nedvetsky et al. 2009). AQP2 is known to be ubiquitinated on K270, although the E3 ligase responsible is unknown (Lee and Kwon 2009). Research has been conducted in attempt to determine the ubiquitin ligase responsible for AQP2 degradation (Lee et al. 2011). Cul3 was not shown to be associated with $\mathrm{AQP} 2$ and it has been suggested that either Cul5 or Nedd4 is responsible for AQP2 ubiquitination (Lee et al. 2011). The ligase involved remains to be determined but ubiquitination of AQP2 is believed to be necessary for its endocytosis and also exocytosis via the formation of a multivesicular body (Lee 89 
and Kwon 2009). It remains a possibility that Cul3 could be involved in this process, but this possibility seems increasingly unlikely as Cul3 was not found associated with AQP2 (Lee et al. 2011).

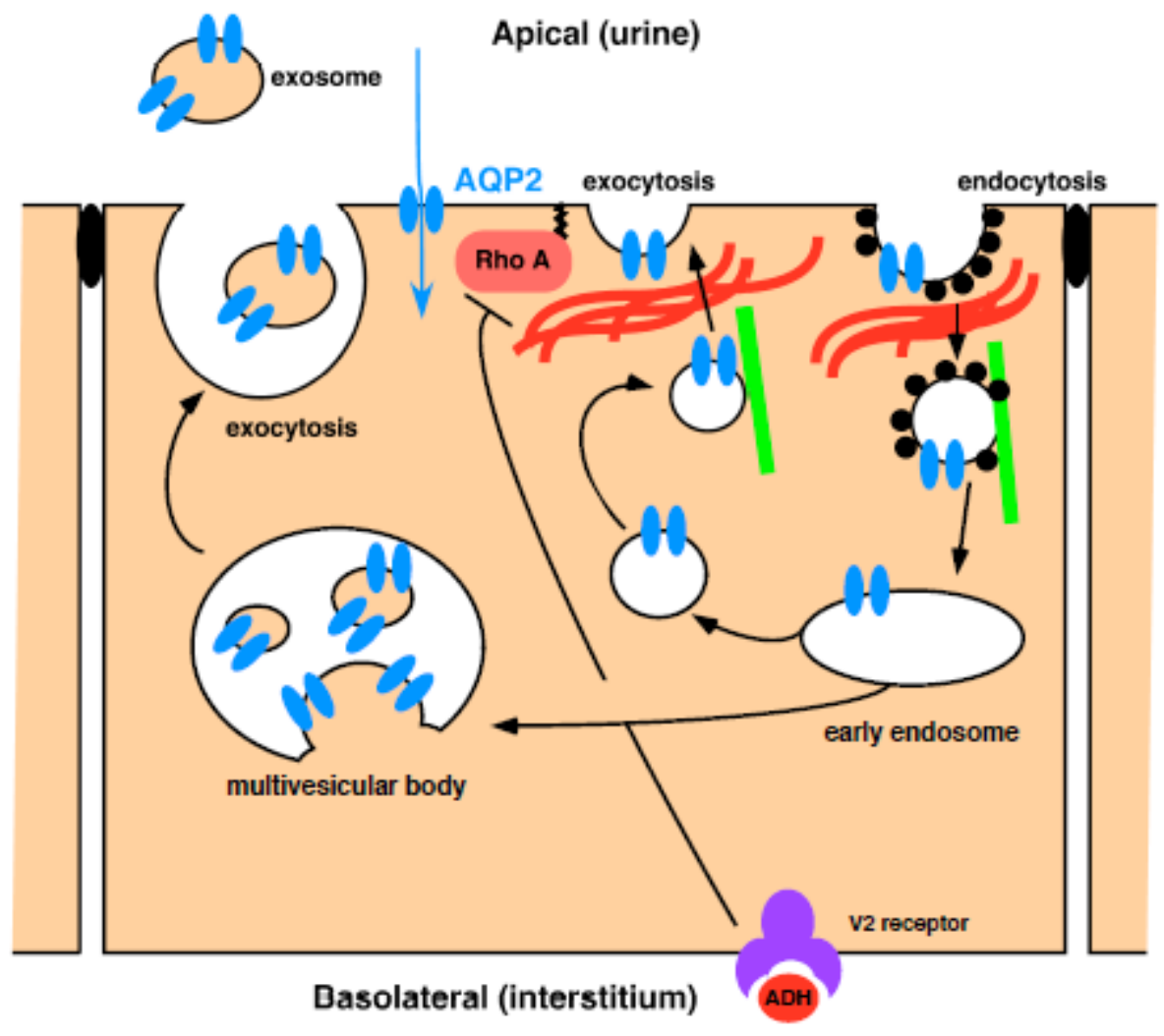

Figure 3.13: Regulation of AQP2 expression at the membrane. This model illustrates the process by which AQP2 is recycled or degraded within the cells of the nephron. This diagram, has been adapted from a review (Takata et al. 2008). 
A possible role for Cul3 in AQP2 regulation in the collecting duct has been recently described via the Keap1-Nrf2 pathway (Suzuki et al. 2017). Nrf2 is a transcription factor involved in the oxidative stress response and it is tightly regulated by the BTB-Kelch protein Keap1 (Wakabayashi et al. 2003). Increased Nrf2 activity during murine development as a result of depleted Keap1 has been shown to result in upregulation of two lectins, Clec4d and Clec4n, that are responsible for the glycosylation of AQP2 (Suzuki et al. 2017). Increased glycosylation of AQP2 results in its increased excretion in exosomes and loss of AQP2 in the collecting duct, resulting in increased urine excretion as a result of the animals' inability to absorb water and a diabetic phenotype (Suzuki et al. 2017). It has been well demonstrated that Nrf2, a Cul3 substrate, regulates AQP2 via this mechanism, but as Cul3 has many other substrates, it is also possible that other Cul3-based mechanisms are also involved in AQP2 regulation.

A second known regulator of AQP2 expression at the apical membrane is RhoA. RhoA is a member of the Rho family of small GTPases and it is known to be involved in the organization of the actin cytoskeleton (Ridley 2001). RhoA is also a known Cul3 substrate, and depletion of Cul3 has been shown to result in increased formation of actin 'stress fibers' in cells (Chen et al 2009). Constitutively active RhoA has been shown to result in the formation of stress fibers and prevent translocation of AQP2 to the apical membrane (Klussmann et al. 2001; Tamma et al. 2001). Therefore, it also remains a possibility that Cul3 regulates AQP2 expression at the membrane at least in part through Cul3-mediated regulation of RhoA. It is possible that increased RhoA activity resulting from loss of Cul3 results in reduced 
translocation of AQP2 to the membrane. One way to investigate this hypothesis would be to measure AQP2 abundance in exosomes of either Cul3 $\mathrm{KO}$ animals or an exosome-producing cell line where either Cul3 or Klhl3 has been depleted.

Regulation of dynactin-mediated transport by Cul3 provides a fourth possible mechanism that might be utilized by Cul3 to regulate AQP2 abundance. Further investigation into a potential role for Klhl3 in p50/dynamitin regulation may help to shed light on this topic. If p50 is indeed regulated in a Cul3-dependent manner, then impaired trafficking of AQP2-containing endosomes may also be a factor that contributes to the reduction in AQP2 that was observed in Cul3 kidney KO animals. Cul3 is known to be involved in other aspects of vesicle trafficking and endosome maturation (Hubner and Peter 2012; Huotari et al. 2012; Gschweitl et al. 2016), so regulation of p50 may not represent the only point at which Cul3 might regulate AQP2 trafficking. Further experimentation in order to increase understanding of the possible role of Klhl3 in trafficking will be necessary in order to determine if Cul3 is regulating AQP2 via endosomal trafficking. It will be important to utilize Klhl3 knockout cells for this experiment instead of Cul3 knockout cells, as Cul3 is already known to have substrates involved in trafficking processes (Hubner and Peter 2012; Gschweitl et al. 2016), and Klhl3 will have fewer substrates than Cul3, thereby allowing for a clearer determination of which pathways might be involved.

In order to begin to determine which of these four mechanisms are responsible for the loss of AQP2 expression in the kidneys of adult Cul3 Kidney KO animals, there are some simple experiments that should be done. First, as it has 92 
been shown that Nrf2 can result in decreased AQP2 expression, which is likely to occur in the Cul3 Pax8 Cre (Kidney KO) animals, it will be necessary to measure Nrf2 expression both in kidneys of adult animals (WT and kidney KO) and in cells or embryos that are hypomorphic or deleted for $\mathrm{Cul} 3\left(\mathrm{Cul}^{\mathrm{fl} \mathrm{fl} / \mathrm{flx}}\right)$ in order to determine if Nrf2 is being regulated by Cul3. As the effects of Nrf2 on AQP2 are a result of Nrf2 levels during embryonic development and not increased Nrf2 levels during adulthood (Suzuki et al. 2017), the results gleaned from experiments with MEFs and floxed (hypomorphic for Cul3 but not Cul3 KO) Cul3 embryos will be the most informative as any increase in Nrf2 will indicate whether or not Cul3 floxed adult animals will suffer from abnormal AQP2 regulation that is Nrf2 dependent. Exosome excretion in Cul3 WT and KO animals, and possibly cell lines, will also be necessary to confirm that the AQP2 is being excreted.

Next, it will be necessary to investigate the contributions that altered levels of RhoA may be having on AQP2 regulation. This can be accomplished by comparing levels of RhoA in WT and Cul3 kidney KO levels by western blot and also immunofluorescence to look for the formation of actin stress fibers, which may be preventing AQP2 from reaching the membrane. Elucidating the possible role of Klhl3 in p50 regulation and endocytic trafficking as was previously discussed will help to determine if Klhl3 plays a role in AQP2 regulation. Lastly, it may be necessary to confirm that Cul3 is not the E3 ligase responsible for AQP2 degradation. As other ligases have already been implicated in this process (Lee and Kwon 2009; Lee et al. 2011), further experimentation may not be necessary. 


\section{Other identified proteins that may interact with Klhl3 ATP1ß1:}

Another protein of interest that was identified in the Klhl3 two-hybrid screens is the beta subunit of the $\mathrm{Na}+\mathrm{K}+\mathrm{ATPase}$, which is expressed in many cell types including the basolateral membrane of the DCT (NCC and ENaC are found on the apical surface). The $\mathrm{Na}+\mathrm{K}+$ ATPase is responsible for maintaining the electrochemical gradient which drives the other renal ion transporters (Hamilton and Devor 2012). ATP1 $\beta 1$ is believed to be ubiquitinated and degraded (Yoshimura et al. 2008).

In addition to its role in renal function, this transporter and its subunits also serve other purposes in different types of cells. ATP1 $\beta 1$ is also associated with cancer, as it plays a role in cell adhesion (Litan and Langhans 2015). Decreased expression of ATP1 $\beta 1$ is associated with poor prognosis in breast cancer patients (Presson et al. 2011). Interestingly, increased expression of TGFßII, which was also identified as a potential Klhl3 binding partner, has also been associated with cancer (Presson et al. 2011). Silencing of ATP1ß1 is also associated with clear cell renal carcinoma (Selvakumar et al. 2014).

Interestingly, the $\mathrm{Na}+\mathrm{K}+\mathrm{ATPase}$ is also very important in brain and may be associated with Down's Syndrome (Lubec and Sohn 2003). ATP1 $\beta 1$ has been shown to be downregulated in scrapie-infected mice (Kim et al. 2008) and also has been implicated in brain pathologies in humans (Brignone et al. 2011). ATP1ß2 (another subunit of this transporter) has also been demonstrated to be important for interactions between neurons and glial cells (Brignone et al. 2011). 
ATP1 1 is a good candidate for a Cul3 and Klhl3 substrate as it has been shown by others that it can be degraded in an ubiquitin-dependent manner (Yoshimura et al. 2008). In order to explore this idea further, ATP1 $\beta 1$ will first need to be cloned into a mammalian expression vector so that it can be checked for binding to Klhl3 and Cul3 as well as used in in vitro ubiquitination assays. As this transporter has been shown to be important in brain as well as kidney, future experiments may seek to investigate the role of Klhl3 or other BTB-Kelch proteins in neuronal development and functioning.

\section{MATERIALS AND METHODS}

Cell culture and transfections: Cells (mDCT, HeLa, HEK293) were grown in DMEM supplemented with ten percent FBS and penicillin/streptomycin as well as lGlutamine. Transfections were conducted using calcium phosphate precipitation. Between 1 and 10 micrograms of DNA was used for each transfection and cells were seeded onto $6 \mathrm{~cm}$ plates. Cells were harvested between 36 and 48 hours posttransfection.

Yeast two-hybrid screens: The two hybrid screens were performed according to the instructions from the Clontech Matchmaker two-hybrid kit (testis and liver screens) and the Clontech Yeast two Hybrid Gold system (Clontech Cat \#630489) for the kidney screen. Full length Klhl3 was cloned into the appropriate bait vector (pGilda for Matchmaker and pGBK47 for Matchmaker Gold). Human testis and fetal liver pB4AD cDNA libraries were purchased from Clontech and screened with the pGilda-Klhl3 bait for the Mathcmaker screens. The pGBK47-Klhl3 bait was used in 
the Matchmaker Gold screen with a pACT2 human kidney cDNA library (Clontech). The sequences of both Klhl3 bait clones were confirmed by Sanger sequencing prior to transformation. The bait vector was then transformed into the appropriate Saccharomyces cerevisiae strain (strain EGY48 for the 'Matchmaker' screens and yeast strain Y2HGold for the 'Matchmaker Gold' screens) followed by transformation of the library. The bait vectors were transformed using electroporation and all library transformations were completed using a lithium acetate transformation procedure. The efficiency of each library transformation (number of clones screened, Table 3.1) was determined by calculating a library titer. Each library plasmid contains a unique cDNA sequence fused to a sequence coding for a DNA activation domain. When a library plasmid interacts with the bait plasmid (Klhl3), then the colony will grow on selective media and turn blue in the presence of a beta galactosidase indicator. Using this system, three screens were performed using human cDNA libraries; Human Matchmaker Kidney, Human Fetal Liver MATCHMAKER LexA cDNA Library, and Human Testis MATCHMAKER LexA cDNA library (Clontech).

Immunofluorescence: Immunofluorescence was performed as previously described in Chapter two as well as previous work from our lab (Cummings et al. 2009).

Western blots and Immunoprecipitations: These were performed as previously described in Chapter 2 as well as previous work from our lab (Wimuttisuk et al. 2014). 
Cloning of p50 and Klhl3: Klhl3 was cloned into the yeast two-hybrid vectors pGilda and pGBKT7 (Clontech) via PCR from a mammalian expression vector followed by restriction digests and ligation into the appropriate yeast 'bait' vector. P50 was cloned by PCR from a human testis cDNA library and inserted into an HAtagged CS2+ mammalian expression vector. Later, p50 was also sub-cloned from the HA-tagged vector into an S-tagged expression vector (CS2+) using restriction digests followed by ligation into the new vector.

\section{REFERENCES}

Boyden LM, Choi M, Choate KA, Nelson-Williams CJ, Farhi A, Toka HR, Tikhonova IR, Bjornson R, Gharavi AG, Goilav B et al. 2012. Mutations in kelch-like 3 and cullin 3 cause hypertension and electrolyte abnormalities. Nature Genetics: 111.

Brignone MS, Lanciotti A, Macioce P, Macchia G, Gaetani M, Aloisi F, Petrucci TC, Ambrosini E. 2011. The beta1 subunit of the Na,K-ATPase pump interacts with megalencephalic leucoencephalopathy with subcortical cysts protein 1 (MLC1) in brain astrocytes: new insights into MLC pathogenesis. Human molecular genetics 20: 90-103.

Burkhardt JK, Echeverri CJ, Nilsson T, Vallee RB. 1997. Overexpression of the dynamitin (p50) subunit of the dynactin complex disrupts dynein-dependent maintenance of membrane organelle distribution. The Journal of cell biology 139: 469-484.

Cummings CM, Bentley CA, Perdue SA, Baas PW, Singer JD. 2009. The Cul3/Klhdc5 E3 ligase regulates p60/katanin and is required for normal mitosis in mammalian cells. The Journal of biological chemistry 284: 11663-11675.

Franken L, Kurts C, Burgdorf S. 2013. Monitoring the intracellular routing of internalized antigens by immunofluorescence microscopy. Methods in molecular biology (Clifton, NJ) 960: 371-377.

Gschweitl M, Ulbricht A, Barnes CA, Enchev RI, Stoffel-Studer I, Meyer-Schaller N, Huotari J, Yamauchi Y, Greber UF, Helenius A et al. 2016. A SPOPL/Cullin-3 ubiquitin ligase complex regulates endocytic trafficking by targeting EPS15 at endosomes. eLife 5: e13841.

Hamilton KL, Devor DC. 2012. Basolateral membrane K+ channels in renal epithelial cells. American journal of physiology Renal physiology 302: F1069-1081. 
Hubner M, Peter M. 2012. Cullin-3 and the endocytic system: New functions of ubiquitination for endosome maturation. Cellular logistics 2: 166-168.

Huotari J, Meyer-Schaller N, Hubner M, Stauffer S, Katheder N, Horvath P, Mancini R, Helenius A, Peter M. 2012. Cullin-3 regulates late endosome maturation. Proceedings of the National Academy of Sciences of the United States of America 109: 823-828.

Ibeawuchi SR, Agbor LN, Quelle FW, Sigmund CD. 2015. Hypertension-causing Mutations in Cullin3 Protein Impair RhoA Protein Ubiquitination and Augment the Association with Substrate Adaptors. The Journal of biological chemistry 290: 19208-19217.

Kim HO, Snyder GP, Blazey TM, Race RE, Chesebro B, Skinner PJ. 2008. Prion disease induced alterations in gene expression in spleen and brain prior to clinical symptoms. Advances and applications in bioinformatics and chemistry : $A A B C$ 1: $29-50$.

Klussmann E, Tamma G, Lorenz D, Wiesner B, Maric K, Hofmann F, Aktories K, Valenti G, Rosenthal W. 2001. An inhibitory role of Rho in the vasopressinmediated translocation of aquaporin-2 into cell membranes of renal principal cells. The Journal of biological chemistry 276: 20451-20457.

Lee YJ, Kwon TH. 2009. Ubiquitination of aquaporin-2 in the kidney. Electrolyte \& blood pressure : $E$ \& BP 7: 1-4.

Lee YJ, Lee JE, Choi HJ, Lim JS, Jung HJ, Baek MC, Frokiaer J, Nielsen S, Kwon TH. 2011. E3 ubiquitin-protein ligases in rat kidney collecting duct: response to vasopressin stimulation and withdrawal. American journal of physiology Renal physiology 301: F883-896.

Litan A, Langhans SA. 2015. Cancer as a channelopathy: ion channels and pumps in tumor development and progression. Frontiers in cellular neuroscience 9: 86.

Louis-Dit-Picard H, Barc J, Trujillano D, Miserey-Lenkei S, Bouatia-Naji N, Pylypenko O, Schott J-J, Jeunemaitre X. 2012. KLHL3 mutations cause familial hyperkalemic hypertension by impairing ion transport in the distal nephron. Nature Genetics 44: 456-460.

Lubec G, Sohn SY. 2003. RNA microarray analysis of channels and transporters in normal and fetal Down syndrome (trisomy 21) brain. Journal of neural transmission Supplementum: 215-224.

Marples D, Schroer TA, Ahrens N, Taylor A, Knepper MA, Nielsen S. 1998. Dynein and dynactin colocalize with AQP2 water channels in intracellular vesicles from kidney collecting duct. The American journal of physiology 274: F384394.

Mathew R, Seiler MP, Scanlon ST, Mao AP, Constantinides MG, Bertozzi-Villa C, Singer JD, Bendelac A. 2012. BTB-ZF factors recruit the E3 ligase cullin 3 to regulate lymphoid effector programs. Nature 491: 618-621.

McCormick JA, Ellison DH. 2011. The WNKs: Atypical Protein Kinases with Pleiotropic Actions. Physiology Reviews 91: 177-219.

McCormick JA, Yang CL, Zhang C, Davidge B, Blankenstein KI, Terker AS, Yarbrough B, Meermeier NP, Park HJ, McCully B et al. 2014. Hyperkalemic hypertension- 
associated cullin 3 promotes WNK signaling by degrading KLHL3. The Journal of clinical investigation 124: 4723-4736.

Nedvetsky PI, Tamma G, Beulshausen S, Valenti G, Rosenthal W, Klussmann E. 2009. Regulation of aquaporin-2 trafficking. Handbook of experimental pharmacology: 133-157.

O'Shaughnessy KM. 2015. Gordon Syndrome: a continuing story. Pediatric nephrology (Berlin, Germany) 30: 1903-1908.

Ohta A, Schumacher F-R, Mehellou Y, Johnson C, Knebel A, Macartney TJ, Wood NT, Alessi DR, Kurz T. 2013. The CUL3-KLHL3 E3 ligse complex mutated in Gordon's hypertension syndrome interacts with and ubiquitylates WNK isoforms: disease causing mutations in Klhle and WNK4 disrupt interaction. Biochemical Journal 451: 111-122.

Olesen ET, Fenton RA. 2017. Aquaporin-2 membrane targeting: still a conundrum. American journal of physiology Renal physiology: ajprenal 0001002017.

Presson AP, Yoon NK, Bagryanova L, Mah V, Alavi M, Maresh EL, Rajasekaran AK, Goodglick L, Chia D, Horvath S. 2011. Protein expression based multimarker analysis of breast cancer samples. BMC cancer 11: 230.

Ridley AJ. 2001. Rho proteins: linking signaling with membrane trafficking. Traffic (Copenhagen, Denmark) 2: 303-310.

Selvakumar P, Owens TA, David JM, Petrelli NJ, Christensen BC, Lakshmikuttyamma A, Rajasekaran AK. 2014. Epigenetic silencing of Na,K-ATPase beta 1 subunit gene ATP1B1 by methylation in clear cell renal cell carcinoma. Epigenetics $\mathbf{9}$ : 579-586.

Shibata S, Zhang J, Puthumana J, Stone KL, Lifton RP. 2013. Kelch-like 3 and Cullin 3 regulate electrolyte homeostasis via ubiquitination and deradation of WNK4. PNAS 110: 7838-7843.

Singer JD, Gurian-West M, Clurman B, Roberts JM. 1999. Cullin-3 targets cyclin E for ubiquitination and controls S phase in mammalian cells. Genes Dev 13: 23752387.

Suzuki T, Seki S, Hiramoto K, Naganuma E, Kobayashi EH, Yamaoka A, Baird L, Takahashi N, Sato H, Yamamoto M. 2017. Hyperactivation of Nrf2 in early tubular development induces nephrogenic diabetes insipidus. Nature communications 8: 14577.

Takata K, Matsuzaki T, Tajika Y, Ablimit A, Hasegawa T. 2008. Localization and trafficking of aquaporin 2 in the kidney. Histochemistry and cell biology $\mathbf{1 3 0}$ 197-209.

Tamma G, Klussmann E, Maric K, Aktories K, Svelto M, Rosenthal W, Valenti G. 2001. Rho inhibits cAMP-induced translocation of aquaporin-2 into the apical membrane of renal cells. American journal of physiology Renal physiology 281: F1092-1101.

Wakabayashi M, Mori T, Isobe K, Sohara E, Susa K, Araki Y, Chiga M, Kikuchi E, Nomura N, Mori Y et al. 2013. Impaired KLHL3-mediated Ubiquitination of WNK4 Causes Human Hypertenison. Cell Reports 2013: 1-11. 
Wakabayashi N, Itoh K, Wakabayashi J, Motohashi H, Noda S, Takahashi S, Imakado S, Kotsuji T, Otsuka F, Roop DR et al. 2003. Keap1-null mutation leads to postnatal lethality due to constitutive Nrf2 activation. Nat Genet 35: 238-245.

Wimuttisuk W, West M, Davidge B, Yu K, Salomon A, Singer JD. 2014. Novel Cul3 binding proteins function to remodel E3 ligase complexes. BMC cell biology 15: 28.

Yang CL, Angell J, Mitchell R, Ellison DH. 2003. WNK kinases regulate thiazidesensitive $\mathrm{Na}-\mathrm{Cl}$ cotransport. The Journal of clinical investigation 111: 10391045.

Yoshimura SH, Iwasaka S, Schwarz W, Takeyasu K. 2008. Fast degradation of the auxiliary subunit of $\mathrm{Na}+/ \mathrm{K}+-\mathrm{ATPase}$ in the plasma membrane of HeLa cells. Journal of cell science 121: 2159-2168. 
CHAPTER 4: Conclusions, Discussion, and Future Directions 
The work presented here highlights the multitude of cellular processes that are dependent on Cul3-based ubiquitin ligase complexes. The first chapter summarizes what is known about the Cul3 ubiquitin ligase including its structure, cellular functions, and the diseases in which Cul3 is known to be involved. In the body of this dissertation has contributed to a greater understanding of the cellular processes that are governed by Cul3. Chapter two describes the mechanism utilized by Cul3 to target cyclin E for degradation while also helping to establish a role for Cul3 in cancer, as low molecular weight cyclin E that cannot be degraded by Cul3 is associated with tumorigenesis. Chapter three explains our understanding of Cul3's role in the kidney where it is involved in ion homeostasis and the maintenance of blood pressure. These findings have led to several new research questions worthy of exploration. This final chapter will help to explain the significance of this work and propose new questions and ideas for future experiments relating to these projects. These projects have led to five new research questions, which will be addressed in this chapter:

1. What is the nature of the interactions between Cul3, Cdk3, and the LRR protein MUF1?

2. How does loss of Cul3 affect breast cancer cells?

3. What other posttranslational modifications might affect cyclin $E$ in vivo?

4. How do BTB proteins affect the binding of E2 enzymes to Cul3based complexes? 


\section{Do changes in Nrf2 activity affect regulation of AQP2 in Cul3 conditional knockout or Cul3 $\Delta 403-459$ mice?}

\section{RESEARCH QUESTION 1: What is the nature of the interactions between Cul3, Cdk2, and the LRR protein MUF1? \\ BACKGROUND: LRR proteins and Cul3}

Cul3 is a member of the Cullin-RING family of ubiquitin ligases, and as such has many structural similarities to other members of the Cullin family. Examples include modification by the ubiquitin-like protein Nedd8, an N-terminal domain which interacts with substrate adaptors, and a C-terminal domain which binds the RING finger protein, Rbx1, which is believed to be responsible for interacting with the E2 enzyme to facilitate substrate ubiquitination (Petroski and Deshaies 2005) . Each cullin interacts with a different set of substrate adaptors in order to recognize substrates (Petroski and Deshaies 2005). For example, Cul1 is known to require two substrate adaptor proteins: the Skp1 linker protein, which binds to both Cul1 and the F-box containing substrate adaptor, for example Fbxw7. Unlike Cul1, Cul3 substrate adaptors are only known to consist of one BTB domain containing protein (Refer to Figure 1.3 in Chapter 1 for a visual comparison) (Xu et al. 2003). For the sake of clarity, it is helpful to note that many of the findings included in this section as background information have been published (Wimuttisuk et al. 2014).

In order to increase our understanding of the Cul3-based complex and identify proteins that might interact with Cul3, a proteomics screen was performed. This screen identified a variety of potential Cul3 interacting proteins including ten proteins which contain leucine-rich repeat (LRR) domains (Wimuttisuk et al. 2014). 
The identification of many LRR proteins in the screen led to the hypothesis that they may play a structural role in Cul3-based complexes in a similar manner that the LRR protein Skp1 plays in Cul1-based complexes (Wimuttisuk et al. 2014). The proteins identified are involved in a variety of cellular processes, from extracellular matrix construction to neuronal structure and function. The information that is known about these ten proteins is summarized in the list below:

1. LRR1/LRRC8B/ TA-LRRP: This protein is a member of the LRRC8 family. It is expressed in many tissues (Kubota et al. 2004).

2. LRR2/PRAME family member 8: Members of the PRAME family are considered to be Cancer testis-antigens based on their expression profiles and can be found in different types of tumors (Wadelin et al. 2010). PRAMElike proteins can be expressed in normal tissues as well (Wadelin et al. 2010).

3. LRR3/SALM-1: This protein is found in axons and dendrites as well as the synaptic membrane (Morimura et al. 2006). SALM 1 (Synaptic adhesion like molecule-1) is a member of the SALM/Lrfn family of neuronal LRR proteins. This protein has been shown to be involved in the formation homo and heteromeric complexes (Nam et al. 2011). Another member of the SALM family, SALM5, may be involved in autism as well as schizophrenia (Nam et al. 2011).

4. LRR4/Caspase recruitment domain protein 7: This protein contains a caspase recruitment domain (CARD). The CARD domain is a member of the death-fold superfamily, which is involved in the formation of signaling 104 
complexes resulting in the activation of caspases and kinases (Kersse et al. 2011).

5. LRR5/Fibromodulin: Fibromodulin is a component of the extracellular matrix. It is expressed in B-cell chronic lymphocytic leukemia and mantle cell lymphoma, but not other blood cancers (Mikaelsson et al. 2005).

6. LRR6 /MHC Class II Transactivator (CIITA): LRR6/CIITA regulates the activation of MHC II and also transcriptionally regulates many target genes (Wu et al. 2009). This protein is believed to be degraded by the ubiquitinproteasome system, but the E3 ligase that may degrade it is unknown (Wu et al. 2009).

7. LRR7/ Reticulon-4 receptor-like 1 precursor, Nogo66 receptor homolog 2, Nogo66 receptor (NgR) related protein 3: LRR7 is related to the Nogo66 receptor, which is involved in axonal growth. However, LRR7 does not bind the same ligands as $\mathrm{NgR}$ and its function is unknown (Barton et al. 2003).

8. LRR8/Erbin(ErB2 interacting protein): Erbin is expressed in sciatic nerves (Tao et al. 2009). Erbin is another neuronal LRR protein expressed in neurons. It has been shown to be involved in myelination (Tao et al. 2009).

9. LRR9 /LRRK1 (Leucine rich repeat kinase 1): LRRK1 is a homolog to LRRK2, a protein shown to be involved in Parkinson's disease. In rats, LRRK1 is expressed in different parts of the rat body and nervous system throughout development (Westerlund et al. 2008). 
10.LRR10/Densin-180 : Densin 180 is a scaffolding protein found in the postsynaptic densities of neurons (Thalhammer et al. 2009). LRR10/Densin-180 and LRR8/Erbin have many structural similarities.

\section{BACKGROUND: LRR5 (FMOD), LRR3 (SALM1) and Cul3}

In order to determine which, if any, Cul3-dependent processes these proteins might play a role, they were cloned into mammalian expression vectors for transfection into cells. Two LRR proteins which were of interest are LRR5 (FMOD or fibromodulin) and LRR3 (also called SALM1) as both were found to interact with BTB domain containing proteins (Wimuttisuk et al. 2014). As fibromodulin is a component of the extracellular matrix (ECM), we sought to determine its localization, as Cul3 is not an extracellular protein. Immunofluorescence of HAtagged LRR5 (FMOD) and Flag-Cul3 shows that the two proteins can co-localize in the cytoplasm, indicating that it is possible for FMOD to play a role inside the cell in addition to its established role in the ECM (Figure 4.1, rows 4 and 5). A second LRR protein, LRR3, can also co-localize with Cul3 in the cytoplasm (Figure 4.1, rows 2 and 3). LRR3 (SALM1) is a neuronal protein that is normally found in neurons and is involved in synapse formation as well as axonal outgrowth (Nam et al. 2011). Cul3 has been associated with neuronal functioning and several neuronal disorders (Codina-Sola et al. 2015; Lin et al. 2015; Wang et al. 2016), so it is reasonable to speculate that Cul3 and LRR3 (SALM1) may serve a function in the synapse that remains unknown. This hypothesis may provide a useful basis for future 
experimentation, but has not been further explored. All further information in this section will focus on LRR5 (fibromodulin) and seek to determine the potential interactions that might occur between LRR5 and the Cul3 complex within living cells.

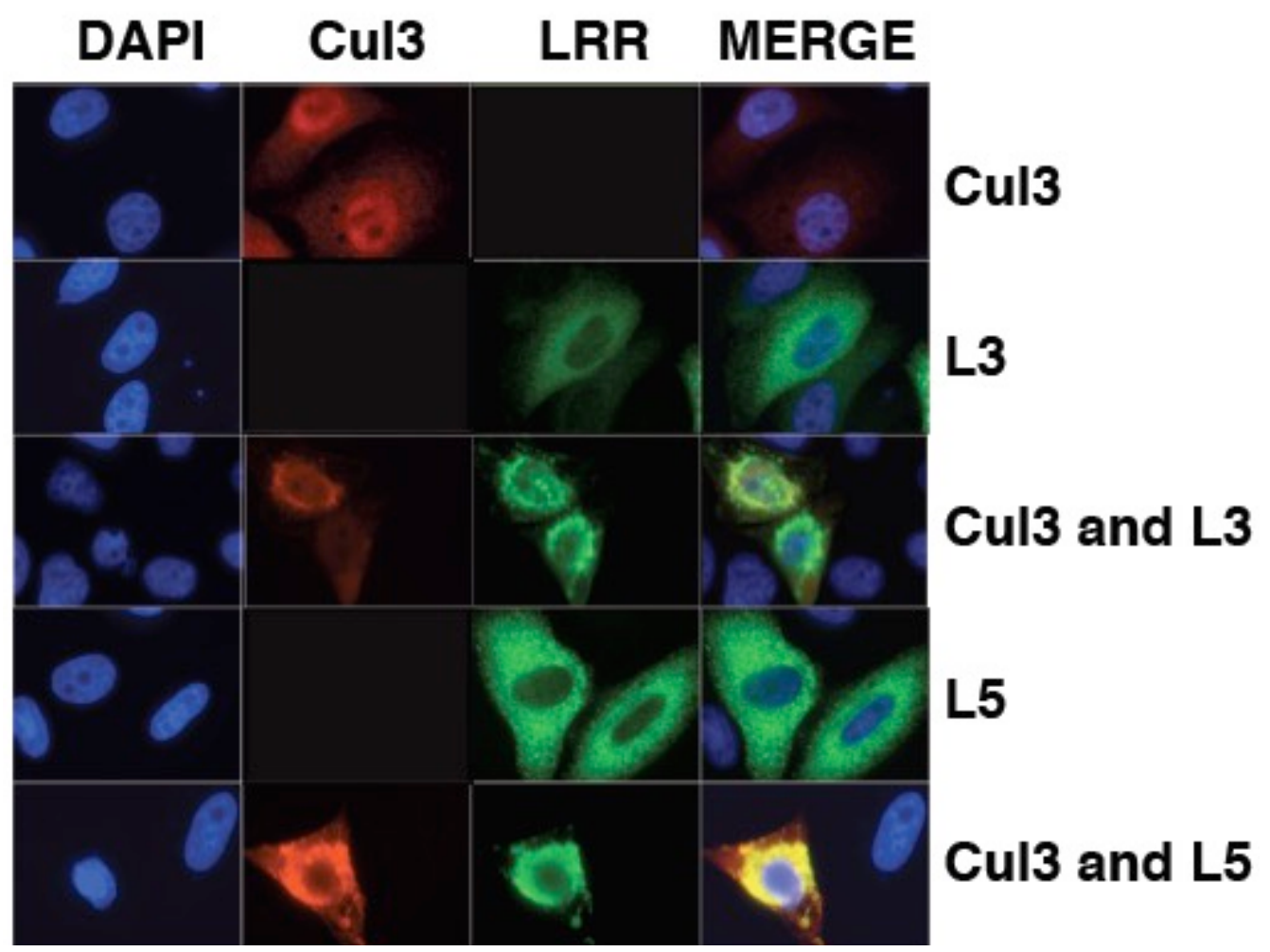

Figure 4.1: Cul3 localizes with LRR proteins. Transfected Flag-Cul3 co-localizes in the cytoplasm with two transfected HA-tagged LRR-domain containing proteins: LRR3 (L3, second row) and LRR5 (L5, bottom row). Antibodies to Flag (Cul3) or HA (LRR proteins) were used for this experiment.

\section{BACKGROUND: LRR5 (FMOD) interacts with the BTB protein FAZF}

In addition to their ability to interact with Cul3, LRR3 and LRR5 can interact with BTB-domain containing proteins (Wimuttisuk et al. 2014). To elucidate the mechanism by which the Cul3 complex might interact with LRR5 within the cell, a 
yeast two-hybrid screen was performed where LRR5 (FMOD) was used as bait and screened agains a human testis cDNA library in order to identify potential LRR5 interacting partners. The only protein that was discovered in this screen was FAZF, a BTB and zinc-finger domain containg protein (Wimuttisuk et al. 2014). FAZF is a transcriptional repressor that is involved in blood cell development and Fanconi anemia, a blood disorder associated with defects in hematopogenesis and bone marrow failure (Dai et al. 2002).

Structurally, FAZF is closely related to PLZF, another DNA binding protein that contains both a BTB domain and a zinc-finger domain (Hoatlin et al. 1999). Further work by our lab revealed that LRR5 can bind FAZF but not PLZF (Wimuttisuk et al. 2014). One structural difference between FAZF and PLZF that we hypothesize might be responsible for the interaction with LRR5 is the presence of a proline-rich region in FAZF which is located just upstream of the BTB domain (Figure 4.2) (Wimuttisuk et al. 2014). This region is not present in PLZF (Wimuttisuk et al. 2014). Analysis of other BTB-domain containing proteins reveals proline rich regions near the BTB domain in Mayven (Klhl2), RhoBTB3, Ctb73, SPOP, and actinfilin (Klhl17). Other proteins such as PLZF and Klhl3 do not contain this region. We hypothesize that this proline-rich region that is located in some BTB proteins may be involved in interactions with LRR-domain containing proteins and that the LRR proteins are not Cul3 substrates and instead might function as a part of the Cul3 complex, possibly as substrate adaptors (Wimuttisuk et al. 2014). Further work will be necessary to determine if proline-rich regions in BTB proteins play a role in any significant interaction between BTB proteins and LRR domain 108 
containing proteins. In order to further explore this idea, several BTB proteins that contain proline rich regions were identified by searching the amino acid sequences of several BTB proteins by eye (Figure 4.2). The identification of these proteins leads us to hypothesize that other pairs of BTB and LRR proteins might exist. One possible pair consists of the BTB protein RhoBTB3 and the LRR protein MUF1.

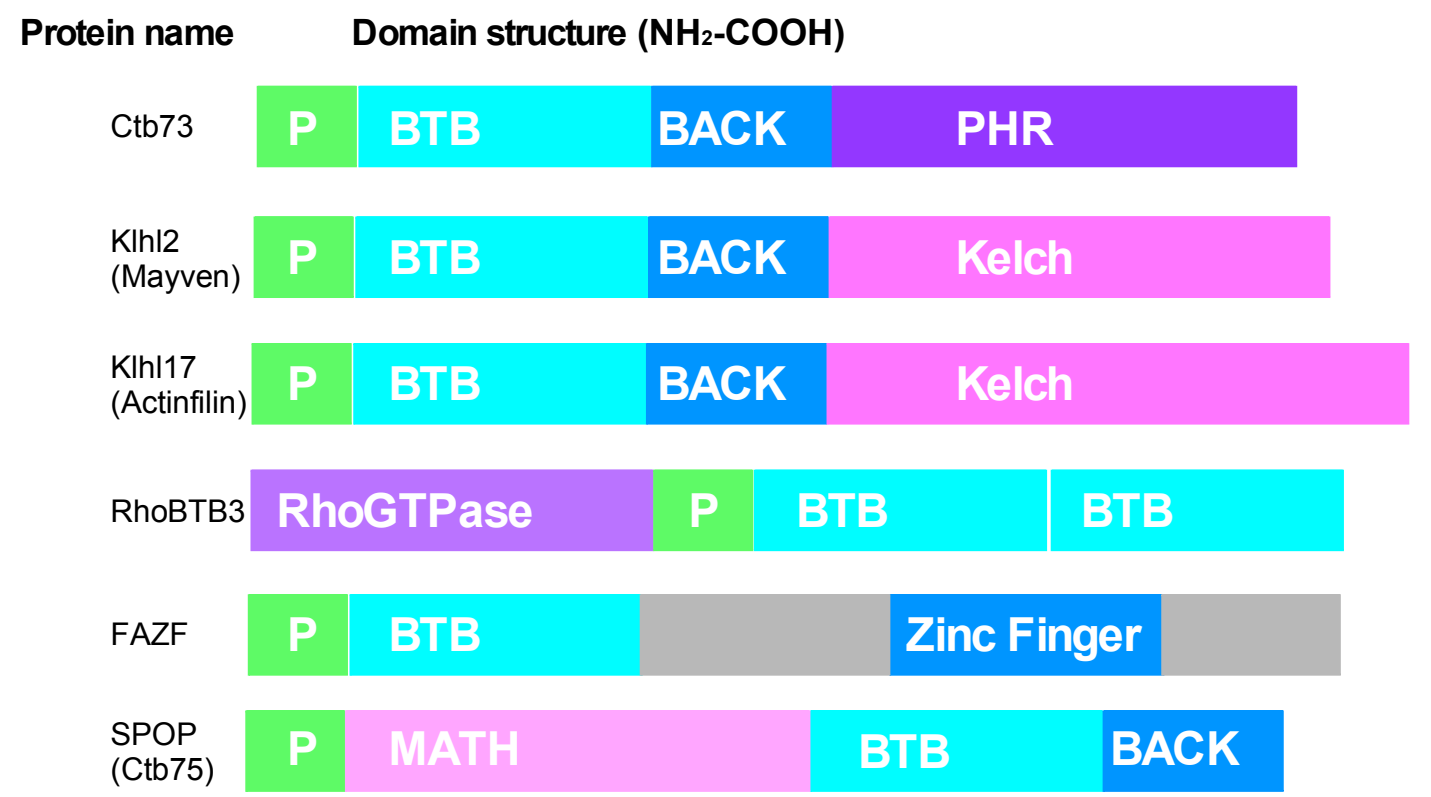

Figure 4.2: Proline-rich regions in BTB proteins. Domain structures of several different BTB proteins show BTB domains located near proline-rich regions. Many BTB proteins contain a proline-rich region, which is located near the $\mathrm{N}$-terminal portion of the BTB domain. Proline-rich regions were identified by looking through the amino acid sequence of each protein to find areas where there are at least 3 prolines located within a ten residue region.

\section{RESULTS: Exploring the interactions between the LRR protein MUF1, Cul3, and Cdk2}

RhoBTB3, a BTB protein with a proline-rich region and two BTB domains (Schenkova et al. 2012), is a member of the RhoBTB family of proteins and also a 
Cul3 substrate adaptor (Schenkova et al. 2012; Lu and Pfeffer 2013; Zhang et al. 2015). RhoBTB3 is of interest as a potential interacting partner for LRR proteins as it has been recently demonstrated that RhoBTB family members, including RhoBTB3, can interact with the LRR protein MUF1(Schenkova et al. 2012). MUF1, also called LRRC41, is a nuclear protein whose function is largely unknown (Schenkova et al. 2012). MUF1 is highly conserved in higher eukaryotes, but has not been the focus of extensive research. Others believe that MUF1 may be a substrate of Cul3 and RhoBTB3 (Schenkova et al. 2012).

MUF1 contains an N-terminal B/C box and is believed to interact with Cul5, and a C-terminal leucine rich repeat (LRR) domain (Kamura et al. 2001). The presence of the B/C box suggests that MUF1 is a Cul5 substrate adaptor, but no substrates of MUF1 are known (Kamura et al. 2001). As RhoBTB family members contain proline-rich regions (Figure 4.2), and have been shown to associate with MUF1, we hypothesized that MUF1 and RhoBTB proteins may interact in a similar way as LRR5 and FAZF. In order to investigate this idea, a FLAG-tagged MUF1 construct was obtained for future experimental use (Kamura et al. 2001).

Two nuclear-localized proteins, cyclin E and MUF1, have been shown to interact with the Golgi-associated RhoBTB3 (Schenkova et al. 2012; Lu and Pfeffer 2013). It has been suggested that both proteins are ubiquitinated by RhoBTB3 at the Golgi, which is peculiar since both proteins are normally localized to the nucleus (Schenkova et al. 2012; Lu and Pfeffer 2013). The amino acid sequences of cyclin E and MUF1 were compared in order to identify any possible similarities between the two proteins (Figure 4.3). Cyclin E contains an MRAIL sequence, which is located 110 
within the cyclin homology domain, and also has the sequence EEIYP further downstream. Both of these sequences are involved in cyclin E's interaction with Cdk2 (Honda et al. 2005). Interestingly, MUF1 contains the sequence RAIV and EEIP further C-terminal, which resembled the Cdk2 interaction region in cyclin E and suggested that MUF1 might also be able to interact with Cdk2. Additionally, MUF1 also contains three possible Cdk2 phosphorylation sites, which suggest that MUF1 may be a substrate of Cdk2 (Figure 4.3).

\begin{abstract}
MUF1:
MEATSREAAPAKSSASGPNAPPALFELCGRAVSAHMGVLESGVWALPGP ILQS ILPLLNIY YLERIEETALKKGLSTQAIWRRLWDELMKTRPSSLESVTCWRAKFMEAFF SHVLRGTIDVS SDRRLCDQRFSPLLHSSRHVRQLTICNMLQGATELVAEPNRRVLETLASSLHTLKFRHLLF SDVAAQQSLRQLLHQLIHHGAVSQVSLYSWPVPESALF ILILTMSAGFWQPGPGGPPCRLC GEASRGRAPSRDEGSLLLGSRRPRRDAAERCAAALMASRRKSEAKQMPRAAPATRVTRRST QESLTAGGTDLKRELHPPATSHEAPGTKRSPSAPAATSSASSSTSSYKRAPASSAPQPKPL KRFKRAAGKKGARTRQGPGAESEDLYDFVFIVAGEKEDGEEMEIGEVACGALDGSDPSCLG LPALEASQRFRSISTLELFTVPLSTEAALTLCHLLSSWVSLESLTLSYNGLGSNIFRLLDS LRALSGQAGCRLRALHLSDLFSPLPILELTRAIVRALPLLRVLSIRVDHPSQRDNPGVPGN AGPPSHIIGDEE I PENCLEQLEMGFPRGAQPAPLLCSVLKASGSLQQLSLDSATFASPQDF GLVLQTLKEYNLALKRLSFHDMNLADCQSEVLFLLQNLTLQEITFSFCRLFEKRPAQFLPE MVAAMKGNSTLKGLRLPGNRLGNAGLLALADVFSEDSSSSLCQLDISSNCIKPDGLLEFAK RLERWGRGAFGHLRLFQNWLDQDAVTAREAIRRLRATCHVVSDSWDSSQAFADYVSTM
\end{abstract}

\begin{abstract}
Cyclin E:
MKEDGGAEFSARSRKRKANVTVFLQDPDEEMAKIDRTARDQCGSQPWDNNAVCADPCSLIP TPDKEDDDRVYPNSTCKPRI IAPSRGSPLPVLSWANREEVWKIMLNKEKTYLRDQHFLEQH PLLQPKMRAILLDWLMEVCEVYKLHRETFYLAQDFFDRYMATQENVVKTLLQLIGISSLF I AAKLEEIYPPKLHQFAYVTDGACSGDEILTMELMIMKALKWRLSPLTIVSWLNVYMQVAYL NDLHEVLLPQYPQQIF IQIAELLDLCVLDVDCLEFPYGILAASALYHFS SSELMQKVSGYQ WCDIENCVKWMVPFAMVIRETGSSKLKHFRGVADEDAHNIQTHRDSLDLLDKARAKKAMLS EQNRASPLPSGLLTPPQSGKKQSSGPEMA
\end{abstract}

Figure 4.3: Sequences of MUF1 and cyclin E. MUF1 has a B/C box, which interacts with Cul5, shown in red. The MRAIL and EEYIP sequences in cyclin E, which interact with Cdk2, are shown in yellow. Similar sequences in MUF1 are also shown in yellow. MUF1 also contains several possible Cdk2 phosphorylation sites (green). The regions shown in pink, located between the RAIL and EEIYP sequences, appear to differ between cyclin E and MUF1. The MUF1 LRR domain is shown in blue and the $\mathrm{B} / \mathrm{C}$ box in red. 


\section{MUF1 binds Cdk2}

The similarities between MUF1 and cyclin E have led us to wonder if MUF1 might be involved in cell cycle regulation. We hypothesized that MUF1 might bind to Cdk2. Binding between Cdk2 and MUF1 was analyzed via immunoprecipitations of transfected protein (Figure 4.4). The results show that MUF1 binds to Cdk2, indicating that Cdk2 and MUF1 might bind in vivo. Further work will be necessary to determine if and how this binding affects the functioning of the cell.

\section{Levels of MUF1 and cyclin E}

There are two possible explanations for the binding between MUF1 and Cdk2: First, it is possible that MUF1 behaves similarly to a cyclin and works with Cdk2 to fulfill a function in the cell, and second, MUF1 may be a substrate of Cdk2. One or both of these situations maybe true. There is a third possibility that can be eliminated: Cdk2 may be a substrate of the Cul5/MUF1 ubiquitin ligase. This is extremely unlikely as Cdk2 is not degraded in a ubiquitin-dependent manner as it is regulated by its cyclin subunits which are rapidly degraded (Felix et al. 1989). Therefore, it is most likely that MUF1 acts as a Cdk2 substrate or binding partner. As cyclin E, a known partner of Cdk2, is an unstable protein, it led us to wonder if cyclin E and MUF1 might compete for Cdk2. We hypothesized that co-transfection of cyclin E and MUF1 may cause changes in the stability of one or both proteins (Figure 4.5)

The results of this experiment show that MUF1 and cyclin E both appear to have diminished levels when co-transfected in the absence of excess Cdk2 (Figure 
4.5, lane 2 compared to others). When Cdk2 is co-transfected and all three proteins are present, however, cyclin E seems to show increased stability and MUF1 shows decreased stability (Figure 4.5 lanes 5 and 6). When HA-Cul3 is co-transfected with Flag-MUF1, it appears that Flag-MUF1 levels may be slightly elevated, which sometimes also seems to be the case when HA-Cdk2 and Flag-MUF1 are cotransfected (Figure 4.5 compare lanes 1, 2, and 3). Taken together, these results indicate an interaction between MUF1, cyclin E, Cdk2, and the Cul3 complex maybe taking place which is altering the stability of MUF1 and cyclin E. As MUF1 contains several possible Cdk2 phosphorylation sites (Figure 4.3), it remains a strong possibility that MUF1 may be a Cdk2 substrate. This interaction may be responsible for regulating levels of MUF1 within the cell. Further experimentation will be necessary to determine if this is the case, and if so the nature of any interaction between MUF1, cyclin E, Cdk2, or Cul3 which may occur. 


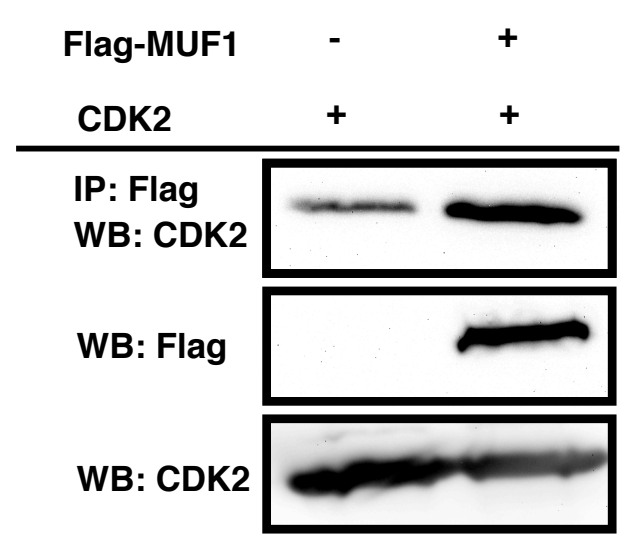

Figure 4.4: MUF1 binds Cdk2. 293 cells were transfected with CDK2 in the presence and absence of Flag-MUF1. The upper blot shows the immunoprecipitation results. The lower blots show protein expression in the cell extracts. 


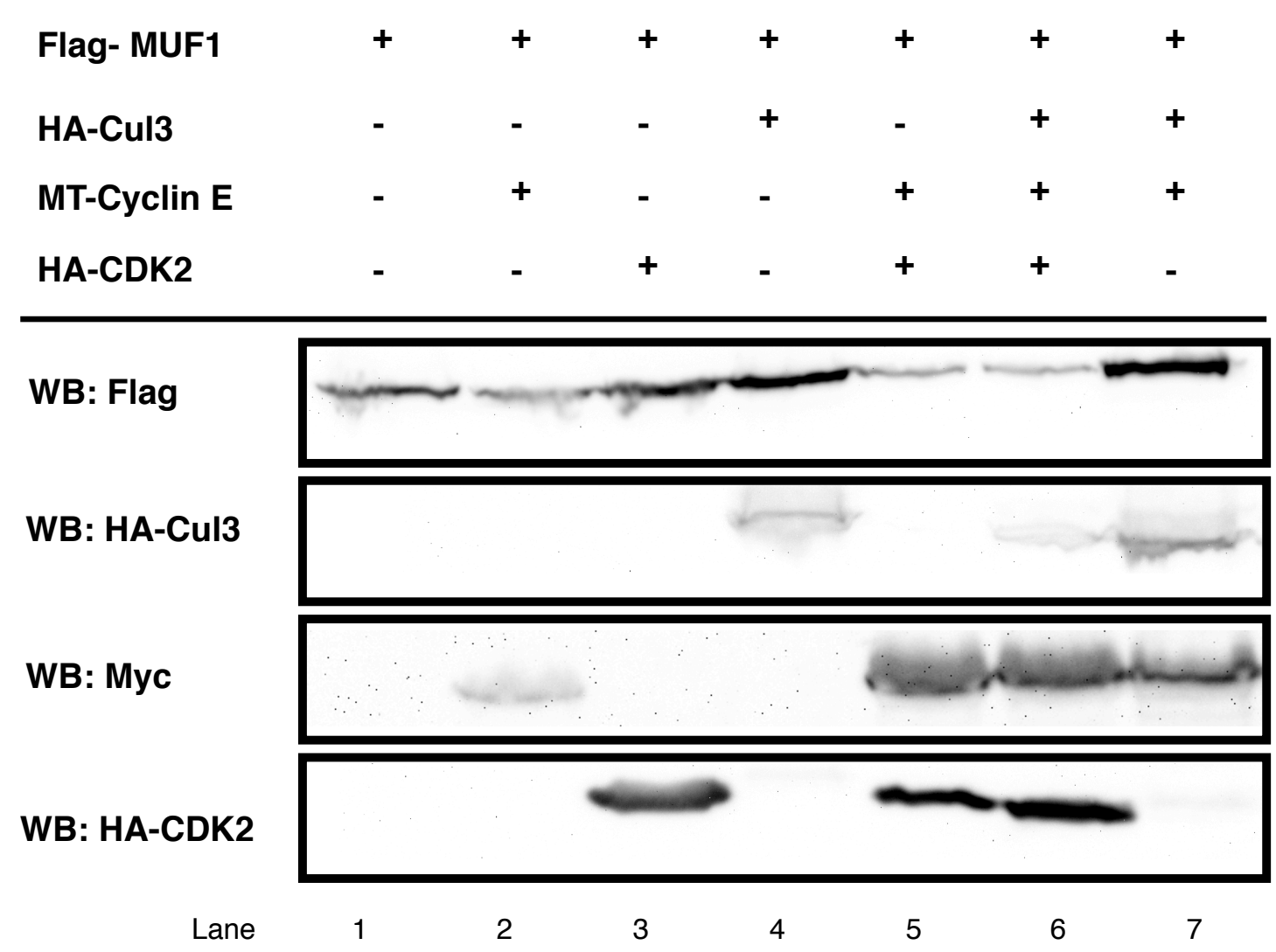

Figure 4.5: Expression levels of transfected MUF1 in the presence of different proteins. Co-transfection and western blotting for Flag-MUF1 or Myc-cyclin was utilized to determine their expression levels in the presence or absence of each other as well as HA-tagged Cul3 or HA-tagged Cdk2. MUF1 is shown in the top panel. Cul3, Myc-cyclin E, and HA-Cdk2 are shown in the three lower blots.

\section{DISCUSSION:}

Previous work from our lab has identified LRR-domain proteins as potential components of the Cul3 complex which might serve as part of multi-subunit substrate adaptors, similar to the system employed by Cul1-based ligases (Wimuttisuk et al. 2014). Interactions have been reported between MUF1 and members of the RhoBTB family including RhoBTB3 (Schenkova et al. 2012). It is possible that these interactions might fit within our published model suggesting that 
LRR proteins can be involved in the formation of Cul3-based complexes via an interaction with BTB domain proteins (Wimuttisuk et al. 2014). This information has led us to our first hypothesis that MUF1 might interact with the RhoBTB proteins via their proline-rich domains (Figure 4.6 panel A).

Although preliminary, the data presented here regarding interactions between MUF1 and the cyclin-dependent kinase Cdk2 suggest that it may play an important role in the cell cycle and merits further study. The information in the literature which demonstrates the ability of RhoBTB3 and related proteins to bind MUF1 as well as the data presented above demonstrating the ability of MUF1 to bind Cdk2 presents a second hypothesis to describe how MUF1 might interact with Cul3 and possibly cyclin E: MUF1 might be a substrate of Cdk2 (Figure 4.6 panel B). MUF1 is a highly conserved protein in mammals (Kamura et al. 2001), indicating that it might be responsible for an important but as of yet unknown function within the cell.

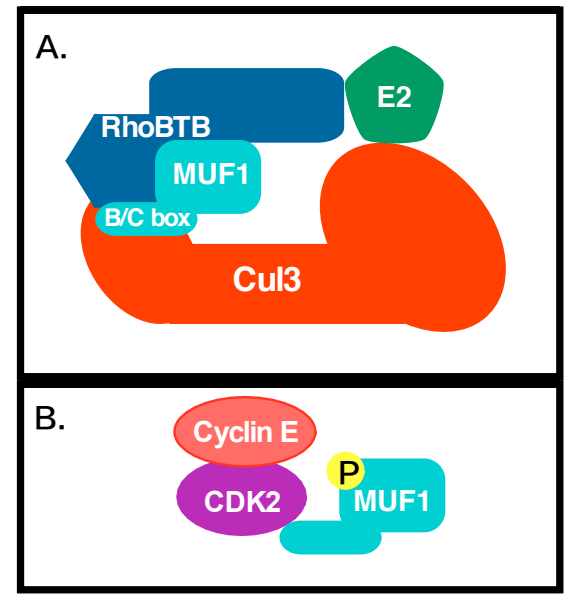

Figure 4.6: Model showing two new hypotheses regarding the potential involvement of MUF1 in regulation of Cul3based complexes. (A) and/or as a possible substrate of Cdk2 (B). MUF1 interacts with proteins in the RhoBTB family, indicated it might associate with Cul3basd complexes via its $\mathrm{B} / \mathrm{C}$ box $(\mathrm{A})$. Other LRR proteins associate with Cul3 in this way (Wimuttisuk, 2014). MUF1 contains two putative Cdk2 phosphorylation sites and may be regulated by Cdk2 (B).

There are many potential future directions for this project as it is a novel idea and still in its infancy. Further study of MUF1's structure and function will require 
experiments to investigate the cellular role of MUF1. MUF1 is a poorly described protein and much information about MUF1 remains unknown, including its tertiary structure and topology. As MUF1 is believed to be a Cul5 substrate adaptor (Kamura et al. 2001), but none of its substrates are known, a yeast two-hybrid screen using MUF1 as bait would be an effective way to identify proteins that might interact with MUF1 in vivo. It is possible that a substrate for MUF1 may be identified by this method. Cell cycle proteins such as Cdk2 and cyclin E may be identified in such a screen. Deletion of MUF1 from mammalian cells would be a second useful method to help to determine its function. This could be accomplished using either siRNA or CRISP-R technology. However, before MUF1 can be deleted it will be necessary to obtain or produce an antibody that can detect endogenous MUF1 and determine which cell types produce MUF1 protein.

In order to address the hypothesis regarding the role of MUF1 and Cdk2, it will be important to identify sequences in MUF1 that facilitate binding to Cdk2. Mutants of the RAIV and EEIP sequences in MUF1 may be useful to determine if MUF1 interacts with Cdk2 in a similar manner as cyclin E. Truncation mutants of MUF1 should be cloned in order to identify which region on MUF1 interacts with Cdk2. As MUF1 also contains several putative Cdk2 phosphorylation sites (Figure 4.3, shown in green), these sites should be mutated to alanines in order to determine if MUF1 is a substrate of Cdk2. Mutants can then be transfected into cells to be checked for binding to Cdk2 and also to help determine if any of the mutants have notable phenotypes. This could be accomplished via fluorescent microscopy and also transfection assays similar to those utilized with cyclin E in chapter two. 
Our original hypothesis, which first led me to MUF1, was that LRR proteins interact with BTB proteins via a proline-rich region on the BTB protein which is located N-terminally of the BTB domain (Figure 4.2). RhoBTB family members possess such a region and have been shown by others to interact with MUF1 (Schenkova et al. 2012). We currently possess a clone of RhoBTB3 in lab, but in order to further investigate this idea it will be necessary to clone RhoBTB1 and RhoBTB2. After cloning all three proteins, the proline rich region should be mutated to alanines in each protein and the resulting mutants should be checked for binding with MUF1 either via immunoprecipitation or yeast two-hybrid screen. These experiments will help to shed light on MUF1's cellular role as well as any involvement it might have in processes that are regulated by Cul3.

\section{RESEARCH QUESTION 2: How does loss of Cul3 affect breast cancer cells?}

Chapter two demonstrated that Cul3 binds cyclin E directly and ubiquitinates its N-terminal domain. This finding is significant given the association between $\mathrm{N}$ terminally truncated cyclin E variants and tumor progression. The next step for this project will be to determine how loss of Cul3 and overexpression of Cul3 affects breast cancer cells to determine how loss of Cul3 might affect cancer progression in humans. Based on the results in chapter two, we hypothesize that breast cancer cells that express LMW cyclin E will have similar amounts of LMW cyclin E protein in cells that contain Cul3 and cells that lack Cul3. In order to test this, human breast cancer cell lines should be edited using the CRISPR Cas-9 system to delete the Cul3 gene in the same manner as it was deleted in the 293 cells that were utilized in 
Chapter two (Ibeawuchi et al. 2015). Three human breast cell lines should be chosen for this experiment; one normal, one cancerous and producing LMW cyclin E, and the third cancerous but not producing LMW cyclin E. Once these three cell lines have been produced, they will be valuable tools and can be compared to their counterparts with wild-type levels of Cul3. The breast cancer cell line MDA-MB-157 has been used by others for the study of LMW cyclin E, so these cells would be an ideal choice (Porter et al. 2001). Based on our own observations the breast cancer cell lines, Sk-br-3, MCF7, and MDA-MB-231 might also be useful for these experiments (Cummings and Singer, unpublished results).

\section{RESEAERCH QUESTION 3: What other modifications may affect cyclin E in vivo?}

Continued work with cyclin E revealed the presence of a band visible in 293 cells and detected with the HE12 cyclin E antibody, which is double the size of the endogenous cyclin E band (Figure 4.7). This band appears to be more prevalent in the Cul3 KO 293 cells (Figure 4.7). Cyclin E is known to undergo several posttranslational modifications, which suggests the possibility that this $90 \mathrm{kDa}$ band might result from an unknown modification. One modification that might result in such a large product is modification by the transglutaminase enzyme TG2. TG2 is known to cross-link glutamine residues with lysine residues in the same protein or lysine residues with lysine residues in other proteins which results in large products and has been previously demonstrated to modify $\mathrm{pRb}$, the retinoblastoma protein, upon cellular stress (Oliverio et al. 1997; Boehm et al. 2002; Mishra et al. 2007). 
Transglutaminase is believed to play a role in apoptosis and also cancer (Mishra et al. 2007).

The cyclin E product that we observed in the 293 cells is large, so we hypothesized that it could be the result of cyclin E modification by TG2. In order to test this, a sample of Cul3 WT and Cul3 KO 293 cells was harvested in lysis buffer and then incubated with varying amounts of TG2 (Figure 4.8). A western blot for cyclin E reveals that with increasing amounts of TG2, both the endogenous $50 \mathrm{kDa}$ and 90kDa cyclin E bands appear to disappear from the gel, whereas levels of endogenous cyclin A remain unchanged (Figure 4.8). This result indicates the possibility that TG is modifying cyclin E but not A. It is possible that the modified cyclin E resulted in a product too large in order to be observed in the ten percent resolving gel that was used for this experiment. In order to determine if cyclin E can be modified in a TG2-dependent manner, this experiment will need to be repeated and the incubated extracts will need to be run on a gradient gel in order to identify any large products that might form. If cyclin E does appear modified in this assay, it will then be necessary to determine if this modification differs between the Cul3 WT and KO cells.

Next, in order to determine if cyclin E can be modified by transfected TG2, either WT or KO 293 cells were transfected with a Myc-tagged human TG2 construct in the presence or absence of the transglutaminase inhibitor dansyl cadaverine (Oliverio et al. 1997). The results of this experiment reveal a $72 \mathrm{kDa}$ band that is detectible with cyclin E antibody, in addition to a smear at the very top of the resolving gel (Figure 4.9). It is possible that this band results from TG2 modification 120 
of cyclin E, but this experiment will need to be repeated to verify these results. Additionally, the dansyl cadaverine did not appear to completely inhibit TG2 activity in this assay, so it will be necessary to titrate the amount of drug that should be added in order to optimize TG2 inhibition (Figure 4.9 lanes 2 and 6).

The results presented here are preliminary and these experiments will need to be optimized and repeated in order to determine if cyclin $\mathrm{E}$ is modified in a transglutaminase-dependent manner. Others have shown the the $\mathrm{Rb}$ protein is modified by transglutaminase during apoptosis (Boehm et al. 2002), so further experimentation suggests that cyclin $\mathrm{E}$ is a transglutaminase substrate, it would be necessary to determine if cyclin E is modified during apoptosis as well.

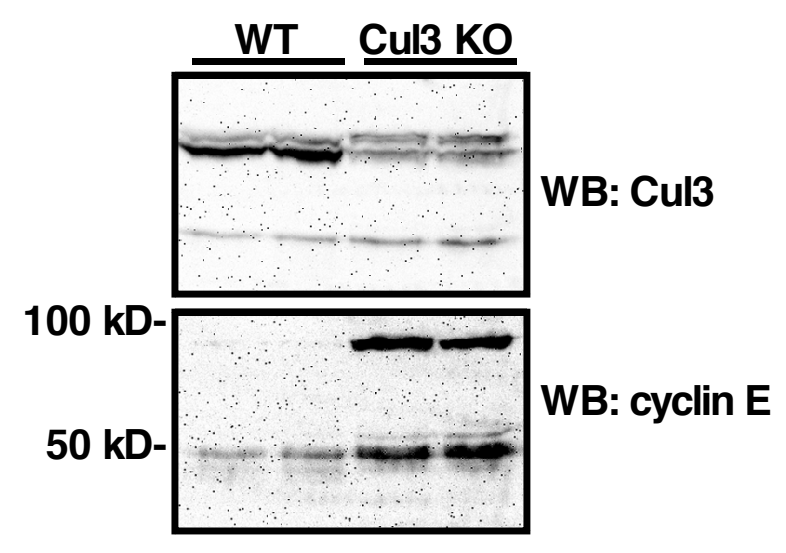

Figure 4.7: $A$ heavy cyclin E band is present in Cul3 KO 293 cells. Western blot showing endogenous levels of Cul3 (top) and cyclin E (bottom) in WT and KO 293 cells. The cyclin E antibody HE12 was used for this experiment. 


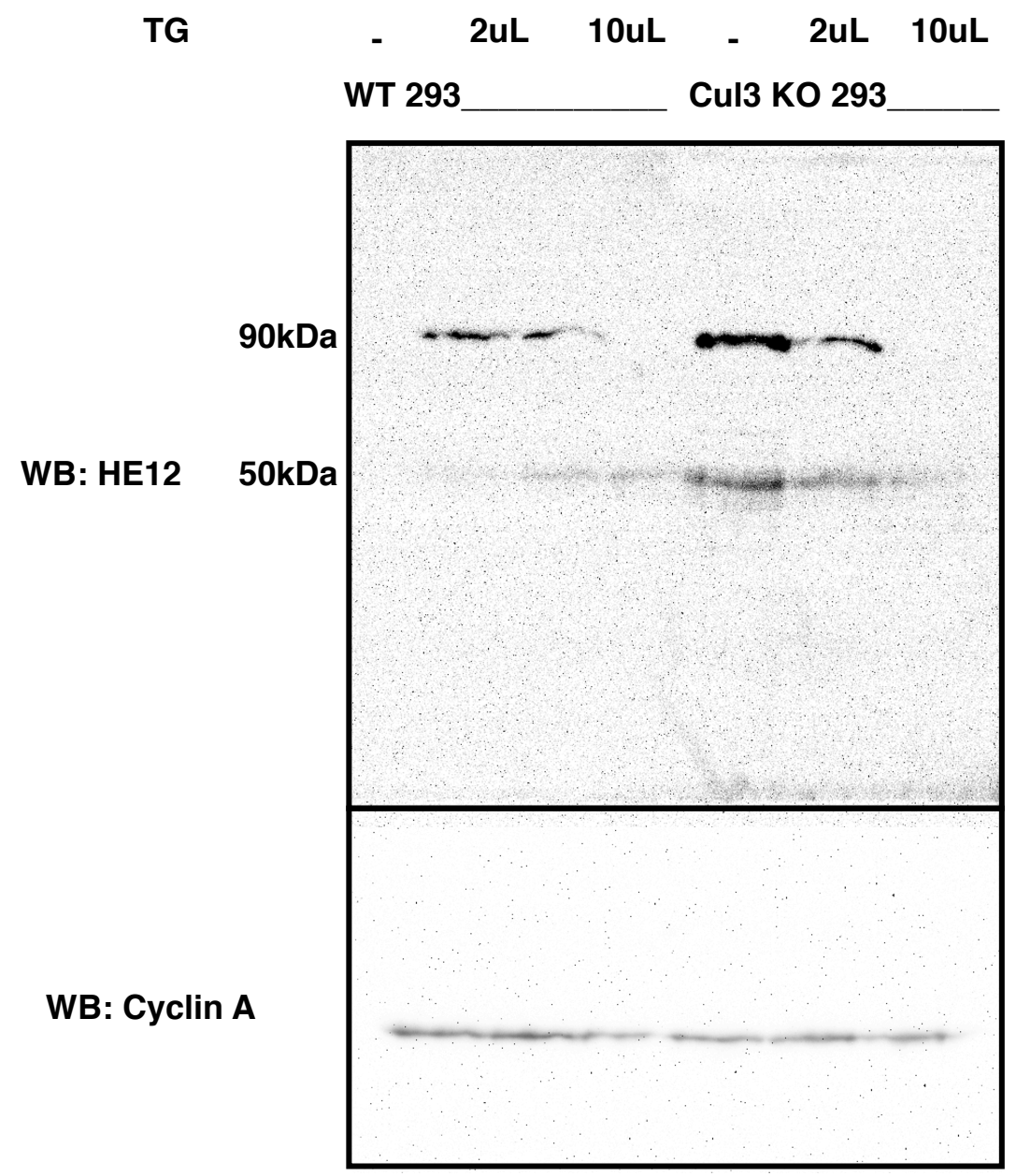

Figure 4.8: The heavy cyclin E band disappears after incubation in transglutaminase. 293 lysates were harvested and incubated in different amounts of transglutaminase for an hour followed by blotting for endogenous cyclin E (top) and cyclin A (bottom). 


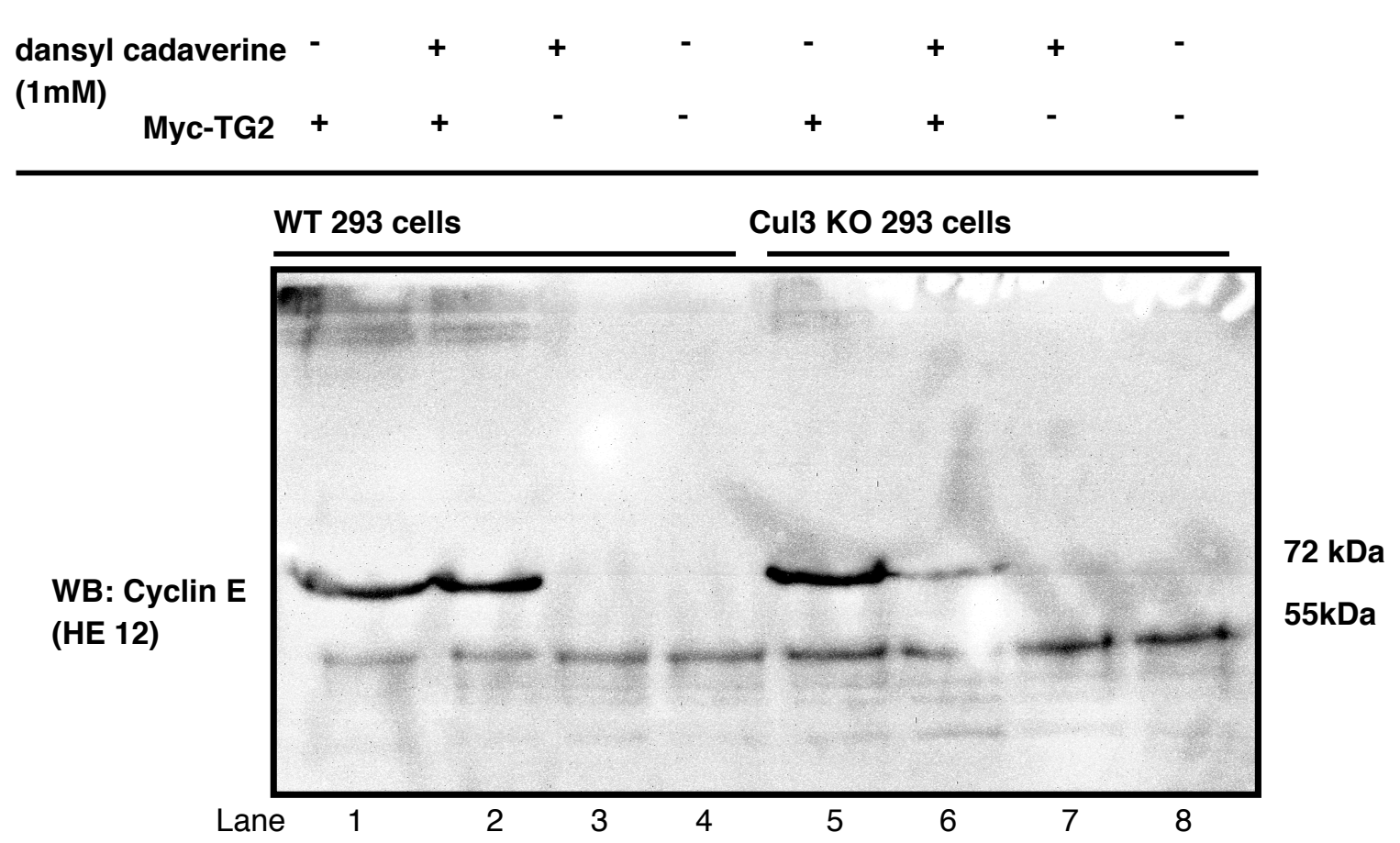

Figure 4.9: The effects of transfected transglutaminase on cyclin E. Either WT (lanes 1 through 4) or Cul3 KO (lanes 5 through 8) 293 cells were transfected with a myctagged transglutaminate (TG2) construct in the presence or absence of the TG inhibitor dansyl cadaverine. A western blot for endogenous cyclin $\mathrm{E}$ is shown.

\section{RESEARCH QUESTION 4: How do BTB proteins affect the binding of E2 enzymes to Cul3-based complexes?}

The data resented in chapter two demonstrated that RhoBTB3, which is believed to be the substrate adaptor for cyclin E, can bind the E2 ubiquitin conjugating enzyme UbE2E1 in the absence of Cul3 (Figure 2.14). Additionally, it was also shown that Cul3 $\Delta 51-67$, which does not bind BTB proteins, cannot interact with UbE2E1 (Figure 2.15). Together, these findings suggest that BTB proteins may be involved in recruitment and binding of E2 enzymes to the Cul3 complex. In order to determine if this is the case, it will be necessary to investigate the interactions between different E2s and BTB proteins. 
One method that will be necessary to explore these interactions is to check the binding of other BTB proteins that are known to result in the degradation of substrates, such as Klhl3 and Keap1, to UbE2E1, as it is known to make K48-linked (degradative) ubiquitin chains (Plafker et al. 2009). This experiment can be conducted by co-transfecting and immunoprecipitating the BTBs with the E2 in both WT and Cul3 KO 293 cells as demonstrated with RhoBTB3 and UbE2E1 (Chapter 2, Figure 2.14). This method would allow for quick detection of interactions between BTB and E2 pairs.

A second method to determine interactions between E2s and BTB proteins would be to perform a yeast two-hybrid screen in which each E2 has been cloned into the 'prey' library vector and each BTB protein is cloned into the 'bait' vector. Once the constructs are completed, they can be transformed into S. cerevisiae and checked for interactions using the Matchmaker Gold two-hybrid system (Clontech). This method would be useful as it would be cost-effective and allow for the screening of many potential BTB-E2 pairs simultaneously.

Next, it will be necessary to explore the interactions between wild-type Cul3 and Cul3 $\Delta 51-67$ with the different E2 enzymes. This can be done easily by using immunoprecipitations to check binding between thee Cul3 constructs and different E2 enzymes. It would be best to complete these experiments using the Cul3 KO 293 cells in order to eliminate the endogenous Cul3 from the binding assays. These experiments will help to further describe the binding between BTB proteins and different E2s as well as helping to identify which E2s might be utilized by Cul3 to facilitate ubiquitination in vivo. This information will help to describe a novel 124 
mechanism by which BTB proteins help to determine the type of ubiquitin chain that is attached to various Cul3 substrates.

\section{RESEARCH QUESTION 5: Do changes in Nrf2 activity affect regulation of AQP2 in Cul3 conditional knockout or Cul3 $\Delta 403-459$ mice?}

One well studied Cul3 substrate is Nrf2 which is involved in the oxidative stress response (Kim and Keum 2016). A recent study utilized a mouse model to show that Nrf2 is responsible for regulating AQP2 abundance in the kidney during development and increased Nrf2 activity during embryonic development results in misregulation of AQP2 and diabetes in adult animals (Suzuki et al. 2017). This is of interest as we found that when Cul3 deletion in the kidneys of adult mice resulted in reduced AQP2 levels (McCormick et al. 2014). As discussed in chapter three, the animals in which we deleted Cul3 were adults, so it is unclear if Nrf2 played a role in the decreased AQP2 expression in this instance. In chapter three, possible roles of Cul3 in AQP2 regulation were discussed, including the effects that increased Nrf2 activity during development might have on AQP2 regulation. This section will focus on a new, two-part, research question related to this topic: How does the hypertension-causing Cul3 mutation (Cul3 $\Delta 403-459)$ affect the levels and activity of the Cul3 substrate Nrf2 during kidney development and AQP2 expression during adulthood?

In order to answer this question, it will be necessary to track kidney development and Nrf2 levels in mouse embryos that are either wild-type for Cul3, deleted or hypomorphic for Cul3, or contain the hypertension-associated mutation. Before measuring the effects of the hypertension mutation (Cul3 $\Delta 403-459$ in 
humans), it will be necessary to track normal kidney development and Nrf2 levels in comparison to embryos that are deficient for Cul3. This extra step will be necessary as the work associating the misregulation of Nrf2 in kidney development was done using a Keap1 (BTB protein) mouse model (Suzuki et al. 2017), so no direct effect of Cul3 Nrf2 in embryonic kidneys has yet been demonstrated.

We have shown that Cul3 $\Delta 403-459$ is capable of binding BTB proteins and ubiquitinating substrates and may be a gain-of function Cul3 mutant (McCormick et al. 2014). However, the effects of Cul3 $\Delta 403-459$ on individual substrates in vivo remain mostly unknown. The mechanism by which Cul3 and the BTB-Kelch protein Keap1 ubiquitinate Nrf2 has been well described, providing a strong framework for a mechanistic comparison between wild-type Cul3 and Cul3s403-459. A Creinducible mouse model for the Cul3 $\Delta 403-459$ (also called Cul3 $\Delta 9$ ) has been developed (Agbor et al. 2016). This mouse model could be used to induce expression of Cul3 $\Delta 9$ in utero to track kidney development in the knock-in mice in comparison to wild-type. Levels of Nrf2 could be measured in order to determine if Cul3-mediated degradation of Nrf2 remains unchanged during development in the Cul3 $\Delta 9$ animals. AQP2 expression in the adult animals would also need to be quantified. If successful, these experiments could shed light on not only the potential role of Cul3 $\Delta 403-459$ in AQP2 regulation in humans but also help to describe the ability of Cul3 $\Delta 403-459$ to ubiquitinate substrates when compared to wild-type Cul3. As previously mentioned, it will be necessary to determine the effects of loss of Cul3 on Nrf2 in mice before conducting determining the effects of the Cul3 $\Delta 403-459$ mutation. As Nrf2 is known to be involved in a variety of 126 
pathological conditions including cancer (Harder et al. 2015; Holmstrom et al. 2016; Kim and Keum 2016; Suzuki et al. 2017), these results could help to understand the disease phenotype that is present in individuals carrying the Cul3 $\Delta 403-459$ mutation.

\section{FINAL THOUGHTS}

Cul3-based E3 ligase complexes are responsible for the regulation of a variety of cellular pathways, many of which are known to have profound effects on the proper function of multicellular organisms. Moving forward, it will be necessary to further clarify the role of Cul3 in these processes in order to better understand the effects that Cul3 has on human physiology. Chapter two describes a degron recognized by Cul3, which is located in the $\mathrm{N}$-terminal region of cyclin E. Future work should attempt to locate Cul3 degrons in other substrates in order to determine if those degrons resemble the one found in cyclin E.

\section{METHODS:}

Yeast two-hybrid screen: The yeast two hybrid screen was performed using LRR5 (fibromodulin) as bait with the Matchmaker yeast two hybrid system, the same system used for one of the Klhl3 screens in Chapter three. The human testis library (also used for chapter 3) was used for this screen.

Immunofluorescence: Immunofluorescence was performed as previously described in both chapter two and previous work from our lab (Cummings et al. 2009). 
Cell culture, transfections, western blots and immunoprecipitations: All cell culture work utilizing HeLa and 293 cell lines was performed as described in Chapters two and three. The Myc-tagged TG construct was a gift from Dr. Robert Sheaff at the University of Tulsa. Dansyl-cadaverine was added to the appropriate cells (Figure 4.9) at a final concentration of $1 \mathrm{mM}$ to inhibit TG activity. For cyclin E experiments involving TG2, the monoclonal cyclin E antibody HE12 (Santa Cruz Biotechnology) was utilized.

Transglutaminase incubation: Untransfected WT and Cul3 KO 293 cells were harvested in Lysis buffer and sonicated. Lysates were then incubated for 15 minutes in a 37 degree water bath with varying amounts of commercial, food-grade transgluaminase enzyme at a concentration of $50 \mathrm{mg} / \mathrm{mL}$. Following incubation, the lysates were mixed with sample buffer, boiled, and ran on 10 percent polyacrylamide gels for western blotting. Western blots were probed using either the monoclonal cyclin E antibody HE-12 (Santa Cruz Biotechnology) or a polyclonal cyclin A antibody (H-432, Santa Cruz Biotechnology). We would like to thank Dr. Robert Sheaff at University of Tulsa for the TG enzyme and protocol.

\section{REFERENCES}

Agbor LN, Ibeawuchi SC, Hu C, Wu J, Davis DR, Keen HL, Quelle FW, Sigmund CD. 2016. Cullin-3 mutation causes arterial stiffness and hypertension through a vascular smooth muscle mechanism. JCI insight 1: e91015.

Barton WA, Liu BP, Tzvetkova D, Jeffrey PD, Fournier AE, Sah D, Cate R, Strittmatter SM, Nikolov DB. 2003. Structure and axon outgrowth inhibitor binding of the Nogo-66 receptor and related proteins. The EMBO journal 22: 3291-3302.

Boehm JE, Singh U, Combs C, Antonyak MA, Cerione RA. 2002. Tissue transglutaminase protects against apoptosis by modifying the tumor suppressor protein $\mathrm{p} 110 \mathrm{Rb}$. The Journal of biological chemistry 277: 2012720130. 
Codina-Sola M, Rodriguez-Santiago B, Homs A, Santoyo J, Rigau M, Aznar-Lain G, Del Campo M, Gener B, Gabau E, Botella MP et al. 2015. Integrated analysis of whole-exome sequencing and transcriptome profiling in males with autism spectrum disorders. Molecular autism 6: 21.

Cummings CM, Bentley CA, Perdue SA, Baas PW, Singer JD. 2009. The Cul3/Klhdc5 E3 ligase regulates p60/katanin and is required for normal mitosis in mammalian cells. The Journal of biological chemistry 284: 11663-11675.

Dai MS, Chevallier N, Stone S, Heinrich MC, McConnell M, Reuter T, Broxmeyer HE, Licht JD, Lu L, Hoatlin ME. 2002. The effects of the Fanconi anemia zinc finger (FAZF) on cell cycle, apoptosis, and proliferation are differentiation stagespecific. The Journal of biological chemistry 277: 26327-26334.

Felix MA, Pines J, Hunt T, Karsenti E. 1989. Temporal regulation of cdc2 mitotic kinase activity and cyclin degradation in cell-free extracts of Xenopus eggs. Journal of cell science Supplement 12: 99-116.

Harder B, Jiang T, Wu T, Tao S, Rojo de la Vega M, Tian W, Chapman E, Zhang DD. 2015. Molecular mechanisms of Nrf2 regulation and how these influence chemical modulation for disease intervention. Biochemical Society transactions 43: 680-686.

Hoatlin ME, Zhi Y, Ball H, Silvey K, Melnick A, Stone S, Arai S, Hawe N, Owen G, Zelent A et al. 1999. A novel BTB/POZ transcriptional repressor protein interacts with the Fanconi anemia group C protein and PLZF. Blood 94: 3737-3747.

Holmstrom KM, Kostov RV, Dinkova-Kostova AT. 2016. The multifaceted role of Nrf2 in mitochondrial function. Current opinion in toxicology 1: 80-91.

Honda R, Lowe ED, Dubinina E, Skamnaki V, Cook A, Brown NR, Johnson LN. 2005. The structure of cyclin E1/CDK2: implications for CDK2 activation and CDK2independent roles. The EMBO journal 24: 452-463.

Ibeawuchi SR, Agbor LN, Quelle FW, Sigmund CD. 2015. Hypertension-causing Mutations in Cullin3 Protein Impair RhoA Protein Ubiquitination and Augment the Association with Substrate Adaptors. The Journal of biological chemistry 290: 19208-19217.

Kamura T, Burian D, Yan Q, Schmidt SL, Lane WS, Querido E, Branton PE, Shilatifard A, Conaway RC, Conaway JW. 2001. Muf1, a novel Elongin BC-interacting leucine-rich repeat protein that can assemble with Cul5 and Rbx1 to reconstitute a ubiquitin ligase. The Journal of biological chemistry 276: 29748-29753.

Kersse K, Verspurten J, Vanden Berghe T, Vandenabeele P. 2011. The death-fold superfamily of homotypic interaction motifs. Trends in biochemical sciences 36: 541-552.

Kim J, Keum YS. 2016. NRF2, a Key Regulator of Antioxidants with Two Faces towards Cancer. Oxidative medicine and cellular longevity 2016: 2746457.

Kubota K, Kim JY, Sawada A, Tokimasa S, Fujisaki H, Matsuda-Hashii Y, Ozono K, Hara J. 2004. LRRC8 involved in B cell development belongs to a novel family of leucine-rich repeat proteins. FEBS letters 564: 147-152.

Lin GN, Corominas R, Lemmens I, Yang X, Tavernier J, Hill DE, Vidal M, Sebat J, Iakoucheva LM. 2015. Spatiotemporal 16p11.2 protein network implicates 
cortical late mid-fetal brain development and KCTD13-Cul3-RhoA pathway in psychiatric diseases. Neuron 85: 742-754.

Lu A, Pfeffer SR. 2013. Golgi-associated RhoBTB3 targets cyclin E for ubiquitylation and promotes cell cycle progression. The Journal of cell biology 203: 233-250. McCormick JA, Yang CL, Zhang C, Davidge B, Blankenstein KI, Terker AS, Yarbrough B, Meermeier NP, Park HJ, McCully B et al. 2014. Hyperkalemic hypertensionassociated cullin 3 promotes WNK signaling by degrading KLHL3. The Journal of clinical investigation 124: 4723-4736.

Mikaelsson E, Danesh-Manesh AH, Luppert A, Jeddi-Tehrani M, Rezvany MR, Sharifian RA, Safaie R, Roohi A, Osterborg A, Shokri F et al. 2005. Fibromodulin, an extracellular matrix protein: characterization of its unique gene and protein expression in B-cell chronic lymphocytic leukemia and mantle cell lymphoma. Blood 105: 4828-4835.

Mishra S, Melino G, Murphy LJ. 2007. Transglutaminase 2 kinase activity facilitates protein kinase A-induced phosphorylation of retinoblastoma protein. The Journal of biological chemistry 282: 18108-18115.

Morimura N, Inoue T, Katayama K, Aruga J. 2006. Comparative analysis of structure, expression and PSD95-binding capacity of Lrfn, a novel family of neuronal transmembrane proteins. Gene 380: 72-83.

Nam J, Mah W, Kim E. 2011. The SALM/Lrfn family of leucine-rich repeat-containing cell adhesion molecules. Seminars in cell \& developmental biology 22: 492498.

Oliverio S, Amendola A, Di Sano F, Farrace MG, Fesus L, Nemes Z, Piredda L, Spinedi A, Piacentini M. 1997. Tissue transglutaminase-dependent posttranslational modification of the retinoblastoma gene product in promonocytic cells undergoing apoptosis. Molecular and cellular biology 17: 6040-6048.

Petroski MD, Deshaies RJ. 2005. Function and regulation of cullin-RING ubiquitin ligases. Nature reviews Molecular cell biology 6: 9-20.

Plafker KS, Singer JD, Plafker SM. 2009. The ubiquitin conjugating enzyme, UbcM2, engages in novel interactions with components of cullin-3 based E3 ligases. Biochemistry 48: 3527-3537.

Porter DC, Zhang N, Danes C, McGahren MJ, Harwell RM, Faruki S, Keyomarsi K. 2001. Tumor-specific proteolytic processing of cyclin $\mathrm{E}$ generates hyperactive lower-molecular-weight forms. Molecular and cellular biology 21: 6254-6269.

Schenkova K, Lutz J, Kopp M, Ramos S, Rivero F. 2012. MUF1/leucine-rich repeat containing 41 (LRRC41), a substrate of RhoBTB-dependent cullin 3 ubiquitin ligase complexes, is a predominantly nuclear dimeric protein. Journal of molecular biology 422: 659-673.

Suzuki T, Seki S, Hiramoto K, Naganuma E, Kobayashi EH, Yamaoka A, Baird L, Takahashi N, Sato H, Yamamoto M. 2017. Hyperactivation of Nrf2 in early tubular development induces nephrogenic diabetes insipidus. Nature communications 8: 14577. 
Tao Y, Dai P, Liu Y, Marchetto S, Xiong WC, Borg JP, Mei L. 2009. Erbin regulates NRG1 signaling and myelination. Proceedings of the National Academy of Sciences of the United States of America 106: 9477-9482.

Thalhammer A, Trinidad JC, Burlingame AL, Schoepfer R. 2009. Densin-180: revised membrane topology, domain structure and phosphorylation status. Journal of neurochemistry 109: 297-302.

Wadelin F, Fulton J, McEwan PA, Spriggs KA, Emsley J, Heery DM. 2010. Leucine-rich repeat protein PRAME: expression, potential functions and clinical implications for leukaemia. Molecular cancer 9: 226.

Wang T, Guo H, Xiong B, Stessman HA, Wu H, Coe BP, Turner TN, Liu Y, Zhao W, Hoekzema $\mathrm{K}$ et al. 2016. De novo genic mutations among a Chinese autism spectrum disorder cohort. Nature communications 7: 13316.

Westerlund M, Belin AC, Anvret A, Bickford P, Olson L, Galter D. 2008. Developmental regulation of leucine-rich repeat kinase 1 and 2 expression in the brain and other rodent and human organs: Implications for Parkinson's disease. Neuroscience 152: 429-436.

Wimuttisuk W, West M, Davidge B, Yu K, Salomon A, Singer JD. 2014. Novel Cul3 binding proteins function to remodel E3 ligase complexes. BMC cell biology 15: 28.

Wu X, Kong X, Luchsinger L, Smith BD, Xu Y. 2009. Regulating the activity of class II transactivator by posttranslational modifications: exploring the possibilities. Molecular and cellular biology 29: 5639-5644.

Xu L, Wei Y, Reboul J, Vaglio P, Shin TH, Vidal M, Elledge SJ, Harper JW. 2003. BTB proteins are substrate-specific adaptors in an SCF-like modular ubiquitin ligase containing CUL-3. Nature 425: 316-321.

Zhang CS, Liu Q, Li M, Lin SY, Peng Y, Wu D, Li TY, Fu Q, Jia W, Wang X et al. 2015. RHOBTB3 promotes proteasomal degradation of HIFalpha through facilitating hydroxylation and suppresses the Warburg effect. Cell research 25: 1025-1042. 


\section{TERMINAL REFERENCE SECTION}

Abbas T, Jha S, Sherman NE, Dutta A. 2007. Autocatalytic phosphorylation of CDK2 at the activating Thr160. Cell Cycle 6: 843-852.

Agbor LN, Ibeawuchi SC, Hu C, Wu J, Davis DR, Keen HL, Quelle FW, Sigmund CD. 2016. Cullin-3 mutation causes arterial stiffness and hypertension through a vascular smooth muscle mechanism. JCI Insight 1: e91015.

Akli S, Zheng PJ, Multani AS, Wingate HF, Pathak S, Zhang N, Tucker SL, Chang S, Keyomarsi K. 2004. Tumor-specific low molecular weight forms of cyclin E induce genomic instability and resistance to p21, p27, and antiestrogens in breast cancer. Cancer Res 64: 3198-3208.

Ayad NG. 2005. CDKs give Cdc6 a license to drive into S phase. Cell 122: 825-827.

Bagheri-Yarmand R, Biernacka A, Hunt KK, Keyomarsi K. 2010. Low molecular weight cyclin $\mathrm{E}$ overexpression shortens mitosis, leading to chromosome missegregation and centrosome amplification. Cancer Res 70: 5074-5084.

Barton WA, Liu BP, Tzvetkova D, Jeffrey PD, Fournier AE, Sah D, Cate R, Strittmatter SM, Nikolov DB. 2003. Structure and axon outgrowth inhibitor binding of the Nogo-66 receptor and related proteins. EMBO J 22: 3291-3302.

Bernassola F, Karin M, Ciechanover A, Melino G. 2008. The HECT family of E3 ubiquitin ligases: multiple players in cancer development. Cancer Cell 14: 1021.

Besson A, Dowdy SF, Roberts JM. 2008. CDK inhibitors: cell cycle regulators and beyond. Dev Cell 14: 159-169.

Bhaskaran N, van Drogen F, Ng HF, Kumar R, Ekholm-Reed S, Peter M, Sangfelt O, Reed SI. 2013. Fbw7alpha and Fbw7gamma collaborate to shuttle cyclin E1 into the nucleolus for multiubiquitylation. Molecular and cellular biology 33: 85-97.

Blagosklonny MV, Pardee AB. 2002. The restriction point of the cell cycle. Cell Cycle 1: 103-110.

Boehm JE, Singh U, Combs C, Antonyak MA, Cerione RA. 2002. Tissue transglutaminase protects against apoptosis by modifying the tumor suppressor protein $\mathrm{p} 110 \mathrm{Rb}$. The Journal of biological chemistry 277: 2012720130.

Boyden LM, Choi M, Choate KA, Nelson-Williams CJ, Farhi A, Toka HR, Tikhonova IR, Bjornson R, Gharavi AG, Goilav B et al. 2012. Mutations in kelch-like 3 and cullin 3 cause hypertension and electrolyte abnormalities. Nature Genetics: 111.

Brignone MS, Lanciotti A, Macioce P, Macchia G, Gaetani M, Aloisi F, Petrucci TC, Ambrosini E. 2011. The beta1 subunit of the Na,K-ATPase pump interacts with megalencephalic leucoencephalopathy with subcortical cysts protein 1 (MLC1) in brain astrocytes: new insights into MLC pathogenesis. Hum Mol Genet 20: 90-103.

Burkhardt JK, Echeverri CJ, Nilsson T, Vallee RB. 1997. Overexpression of the dynamitin (p50) subunit of the dynactin complex disrupts dynein-dependent maintenance of membrane organelle distribution.J Cell Biol 139: 469-484. 
Chavez-Canales M, Zhang C, Soukaseum C, Moreno E, Pacheco-Alvarez D, VidalPetiot E, Castaneda-Bueno M, Vazquez N, Rojas-Vega L, Meermeier NP et al. 2014. WNK-SPAK-NCC cascade revisited: WNK1 stimulates the activity of the $\mathrm{Na}-\mathrm{Cl}$ cotransporter via SPAK, an effect antagonized by WNK4. Hypertension 64: 1047-1053.

Chen HY, Chen RH. 2016. Cullin 3 Ubiquitin Ligases in Cancer Biology: Functions and Therapeutic Implications. Front Oncol 6: 113.

Cheung TH, Rando TA. 2013. Molecular regulation of stem cell quiescence. Nat Rev Mol Cell Biol 14: 329-340.

Choi YM, Kim KB, Lee JH, Chun YK, An IS, An S, Bae S. 2016. DBC2/RhoBTB2 functions as a tumor suppressor protein via Musashi-2 ubiquitination in breast cancer. Oncogene.

Chou PY, Fasman GD. 1975. The conformation of glucagon: predictions and consequences. Biochemistry 14: 2536-2541.

Clurman BE, Sheaff RJ, Thress K, Groudine M, Roberts JM. 1996. Turnover of cyclin E by the ubiquitin-proteasome pathway is regulated by cdk2 binding and cyclin phosphorylation. Genes Dev 10: 1979-1990.

Codina-Sola M, Rodriguez-Santiago B, Homs A, Santoyo J, Rigau M, Aznar-Lain G, Del Campo M, Gener B, Gabau E, Botella MP et al. 2015. Integrated analysis of whole-exome sequencing and transcriptome profiling in males with autism spectrum disorders. Mol Autism 6: 21.

Cole AJ, Clifton-Bligh R, Marsh DJ. 2015. Histone H2B monoubiquitination: roles to play in human malignancy. Endocr Relat Cancer 22: T19-33.

Coppieters F, Ascari G, Dannhausen K, Nikopoulos K, Peelman F, Karlstetter M, Xu M, Brachet C, Meunier I, Tsilimbaris MK et al. 2016. Isolated and Syndromic Retinal Dystrophy Caused by Biallelic Mutations in RCBTB1, a Gene Implicated in Ubiquitination. Am J Hum Genet 99: 470-480.

Cullinan SB, Gordan JD, Jin J, Harper JW, Diehl JA. 2004. The Keap1-BTB protein is an adaptor that bridges Nrf2 to a Cul3-based E3 ligase: oxidative stress sensing by a Cul3-Keap1 ligase. Molecular and cellular biology 24: 8477-8486.

Cummings CM, Bentley CA, Perdue SA, Baas PW, Singer JD. 2009. The Cul3/Klhdc5 E3 ligase regulates p60/katanin and is required for normal mitosis in mammalian cells. The Journal of biological chemistry 284: 11663-11675.

Dai MS, Chevallier N, Stone S, Heinrich MC, McConnell M, Reuter T, Broxmeyer HE, Licht JD, Lu L, Hoatlin ME. 2002. The effects of the Fanconi anemia zinc finger (FAZF) on cell cycle, apoptosis, and proliferation are differentiation stagespecific. The Journal of biological chemistry 277: 26327-26334.

Delk NA, Hunt KK, Keyomarsi K. 2009. Altered subcellular localization of tumorspecific cyclin $\mathrm{E}$ isoforms affects cyclin-dependent kinase 2 complex formation and proteasomal regulation. Cancer Res 69: 2817-2825.

Duda DM, Borg LA, Scott DC, Hunt HW, Hammel M, Schulman BA. 2008. Structural insights into NEDD8 activation of cullin-RING ligases: conformational control of conjugation. Cell 134: 995-1006.

Duong MT, Akli S, Wei C, Wingate HF, Liu W, Lu Y, Yi M, Mills GB, Hunt KK, Keyomarsi K. 2012. LMW-E/CDK2 deregulates acinar morphogenesis, 
induces tumorigenesis, and associates with the activated b-Raf-ERK1/2mTOR pathway in breast cancer patients. PLoS Genet 8: e1002538.

Ekholm SV, Reed SI. 2000. Regulation of G(1) cyclin-dependent kinases in the mammalian cell cycle. Curr Opin Cell Biol 12: 676-684.

Felix MA, Pines J, Hunt T, Karsenti E. 1989. Temporal regulation of cdc2 mitotic kinase activity and cyclin degradation in cell-free extracts of Xenopus eggs. $J$ Cell Sci Suppl 12: 99-116.

Finley D, Ozkaynak E, Varshavsky A. 1987. The yeast polyubiquitin gene is essential for resistance to high temperatures, starvation, and other stresses. Cell 48: 1035-1046.

Ford C, Chevalier S. 1995. DNA replication. Almost licensed. Curr Biol 5: 1009-1012.

Franken L, Kurts C, Burgdorf S. 2013. Monitoring the intracellular routing of internalized antigens by immunofluorescence microscopy. Methods Mol Biol 960: 371-377.

Furukawa M, Xiong Y. 2005. BTB protein Keap1 targets antioxidant transcription factor Nrf2 for ubiquitination by the Cullin 3-Roc1 ligase. Molecular and cellular biology 25: 162-171.

Geng Y, Yu Q, Sicinska E, Das M, Schneider JE, Bhattacharya S, Rideout WM, Bronson RT, Gardner H, Sicinski P. 2003. Cyclin E ablation in the mouse. Cell 114: 431443.

Genschik P, Sumara I, Lechner E. 2013. The emerging family of CULLIN3-RING ubiquitin ligases (CRL3s): cellular functions and disease implications. EMBOJ 32: 2307-2320.

Geyer R, Wee S, Anderson S, Yates J, Wolf DA. 2003. BTB/POZ domain proteins are putative substrate adaptors for cullin 3 ubiquitin ligases. Mol Cell 12: 783790.

Golbang AP, Cope G, Hamad A, Murthy M, Liu CH, Cuthbert AW, O'Shaughnessy K M. 2006. Regulation of the expression of the $\mathrm{Na} / \mathrm{Cl}$ cotransporter by WNK4 and WNK1: evidence that accelerated dynamin-dependent endocytosis is not involved. Am J Physiol Renal Physiol 291: F1369-1376.

Gonen H, Stancovski I, Shkedy D, Hadari T, Bercovich B, Bengal E, Mesilati S, AbuHatoum O, Schwartz AL, Ciechanover A. 1996. Isolation, characterization, and partial purification of a novel ubiquitin-protein ligase, E3. Targeting of protein substrates via multiple and distinct recognition signals and conjugating enzymes. The Journal of biological chemistry 271: 302-310.

Grice GL, Nathan JA. 2016. The recognition of ubiquitinated proteins by the proteasome. Cell Mol Life Sci 73: 3497-3506.

Gschweitl M, Ulbricht A, Barnes CA, Enchev RI, Stoffel-Studer I, Meyer-Schaller N, Huotari J, Yamauchi Y, Greber UF, Helenius A et al. 2016. A SPOPL/Cullin-3 ubiquitin ligase complex regulates endocytic trafficking by targeting EPS15 at endosomes. Elife 5: e13841.

Haas AL, Rose IA. 1982. The mechanism of ubiquitin activating enzyme. A kinetic and equilibrium analysis. The Journal of biological chemistry 257: 1032910337. 
Haas AL, Warms JV, Hershko A, Rose IA. 1982. Ubiquitin-activating enzyme. Mechanism and role in protein-ubiquitin conjugation. The Journal of biological chemistry 257: 2543-2548.

Hamilton KL, Devor DC. 2012. Basolateral membrane K+ channels in renal epithelial cells. Am J Physiol Renal Physiol 302: F1069-1081.

Hao B, Oehlmann S, Sowa ME, Harper JW, Pavletich NP. 2007. Structure of a Fbw7Skp1-cyclin E complex: multisite-phosphorylated substrate recognition by SCF ubiquitin ligases. Mol Cell 26: 131-143.

Harder B, Jiang T, Wu T, Tao S, Rojo de la Vega M, Tian W, Chapman E, Zhang DD. 2015. Molecular mechanisms of Nrf2 regulation and how these influence chemical modulation for disease intervention. Biochem Soc Trans 43: 680686.

Harwell RM, Porter DC, Danes C, Keyomarsi K. 2000. Processing of cyclin E differs between normal and tumor breast cells. Cancer Res 60: 481-489.

Hicke L. 2001. Protein regulation by monoubiquitin. Nat Rev Mol Cell Biol 2: 195201.

Hoatlin ME, Zhi Y, Ball H, Silvey K, Melnick A, Stone S, Arai S, Hawe N, Owen G, Zelent A et al. 1999. A novel BTB/POZ transcriptional repressor protein interacts with the Fanconi anemia group C protein and PLZF. Blood 94: 3737-3747.

Holmstrom KM, Kostov RV, Dinkova-Kostova AT. 2016. The multifaceted role of Nrf2 in mitochondrial function. Curr Opin Toxicol 1: 80-91.

Honda R, Lowe ED, Dubinina E, Skamnaki V, Cook A, Brown NR, Johnson LN. 2005. The structure of cyclin E1/CDK2: implications for CDK2 activation and CDK2independent roles. EMBO J 24: 452-463.

Hoorn EJ, Ellison DH. 2012. WNK kinases and the kidney. Exp Cell Res 318: 10201026.

Hori T, Osaka F, Chiba T, Miyamoto C, Okabayashi K, Shimbara N, Kato S, Tanaka K. 1999. Covalent modification of all members of human cullin family proteins by NEDD8. Oncogene 18: 6829-6834.

Hubner M, Peter M. 2012. Cullin-3 and the endocytic system: New functions of ubiquitination for endosome maturation. Cell Logist 2: 166-168.

Huotari J, Meyer-Schaller N, Hubner M, Stauffer S, Katheder N, Horvath P, Mancini R, Helenius A, Peter M. 2012. Cullin-3 regulates late endosome maturation. Proc Natl Acad Sci U S A 109: 823-828.

Ibeawuchi SR, Agbor LN, Quelle FW, Sigmund CD. 2015. Hypertension-causing Mutations in Cullin3 Protein Impair RhoA Protein Ubiquitination and Augment the Association with Substrate Adaptors. The Journal of biological chemistry 290: 19208-19217.

Itoh K, Wakabayashi N, Katoh Y, Ishii T, Igarashi K, Engel JD, Yamamoto M. 1999. Keap1 represses nuclear activation of antioxidant responsive elements by Nrf2 through binding to the amino-terminal Neh2 domain. Genes Dev 13: 7686.

Jiang J. 2006. Regulation of Hh/Gli signaling by dual ubiquitin pathways. Cell Cycle 5: 2457-2463. 
Jin J, Harper JW. 2002. RING finger specificity in SCF-driven protein destruction. Dev Cell 2: 685-687.

Johnson JL, Lu C, Raharjo E, McNally K, McNally FJ, Mains PE. 2009. Levels of the ubiquitin ligase substrate adaptor MEL-26 are inversely correlated with MEI$1 /$ katanin microtubule-severing activity during both meiosis and mitosis. Dev Biol 330: 349-357.

Kamura T, Burian D, Yan Q, Schmidt SL, Lane WS, Querido E, Branton PE, Shilatifard A, Conaway RC, Conaway JW. 2001. Muf1, a novel Elongin BC-interacting leucine-rich repeat protein that can assemble with Cul5 and Rbx1 to reconstitute a ubiquitin ligase. The Journal of biological chemistry 276: 29748-29753.

Kawakami T, Chiba T, Suzuki T, Iwai K, Yamanaka K, Minato N, Suzuki H, Shimbara N, Hidaka Y, Osaka F et al. 2001. NEDD8 recruits E2-ubiquitin to SCF E3 ligase. EMBO J 20: 4003-4012.

Keck JM, Summers MK, Tedesco D, Ekholm-Reed S, Chuang LC, Jackson PK, Reed SI. 2007. Cyclin $\mathrm{E}$ overexpression impairs progression through mitosis by inhibiting APC(Cdh1). J Cell Biol 178: 371-385.

Kelly BL, Wolfe KG, Roberts JM. 1998. Identification of a substrate-targeting domain in cyclin E necessary for phosphorylation of the retinoblastoma protein. Proc Natl Acad Sci U S A 95: 2535-2540.

Kersse K, Verspurten J, Vanden Berghe T, Vandenabeele P. 2011. The death-fold superfamily of homotypic interaction motifs. Trends Biochem Sci 36: 541552.

Kim HO, Snyder GP, Blazey TM, Race RE, Chesebro B, Skinner PJ. 2008. Prion disease induced alterations in gene expression in spleen and brain prior to clinical symptoms. Adv Appl Bioinform Chem 1: 29-50.

Kim J, Keum YS. 2016. NRF2, a Key Regulator of Antioxidants with Two Faces towards Cancer. Oxid Med Cell Longev 2016: 2746457.

Kleiger G, Hao B, Mohl DA, Deshaies RJ. 2009. The acidic tail of the Cdc34 ubiquitinconjugating enzyme functions in both binding to and catalysis with ubiquitin ligase SCFCdc4. The Journal of biological chemistry 284: 36012-36023.

Klussmann E, Tamma G, Lorenz D, Wiesner B, Maric K, Hofmann F, Aktories K, Valenti G, Rosenthal W. 2001. An inhibitory role of Rho in the vasopressinmediated translocation of aquaporin-2 into cell membranes of renal principal cells. The Journal of biological chemistry 276: 20451-20457.

Kobayashi A, Kang MI, Okawa H, Ohtsuji M, Zenke Y, Chiba T, Igarashi K, Yamamoto M. 2004. Oxidative stress sensor Keap1 functions as an adaptor for Cul3based E3 ligase to regulate proteasomal degradation of Nrf2. Molecular and cellular biology 24: 7130-7139.

Koepp DM, Schaefer LK, Ye X, Keyomarsi K, Chu C, Harper JW, Elledge SJ. 2001. Phosphorylation-dependent ubiquitination of cyclin E by the SCFFbw7 ubiquitin ligase. Science (New York, NY) 294: 173-177.

Koff A, Cross F, Fisher A, Schumacher J, Leguellec K, Philippe M, Roberts JM. 1991. Human cyclin E, a new cyclin that interacts with two members of the CDC2 gene family. Cell 66: 1217-1228. 
Koff A, Giordano A, Desai D, Yamashita K, Harper JW, Elledge S, Nishimoto T, Morgan DO, Franza BR, Roberts JM. 1992. Formation and activation of a cyclin E-cdk2 complex during the G1 phase of the human cell cycle. Science (New York, NY) 257: 1689-1694.

Kubota K, Kim JY, Sawada A, Tokimasa S, Fujisaki H, Matsuda-Hashii Y, Ozono K, Hara J. 2004. LRRC8 involved in B cell development belongs to a novel family of leucine-rich repeat proteins. FEBS Lett 564: 147-152.

Kwon JE, La M, Oh KH, Oh YM, Kim GR, Seol JH, Baek SH, Chiba T, Tanaka K, Bang OS et al. 2006. BTB domain-containing speckle-type POZ protein (SPOP) serves as an adaptor of Daxx for ubiquitination by Cul3-based ubiquitin ligase. The Journal of biological chemistry 281: 12664-12672.

Lee YJ, Kwon TH. 2009. Ubiquitination of aquaporin-2 in the kidney. Electrolyte Blood Press 7: 1-4.

Lee YJ, Lee JE, Choi HJ, Lim JS, Jung HJ, Baek MC, Frokiaer J, Nielsen S, Kwon TH. 2011. E3 ubiquitin-protein ligases in rat kidney collecting duct: response to vasopressin stimulation and withdrawal. Am J Physiol Renal Physiol 301: F883-896.

Leinonen HM, Kansanen E, Polonen P, Heinaniemi M, Levonen AL. 2015. Dysregulation of the Keap1-Nrf2 pathway in cancer. Biochem Soc Trans 43: 645-649.

Libertini SJ, Robinson BS, Dhillon NK, Glick D, George M, Dandekar S, Gregg JP, Sawai E, Mudryj M. 2005. Cyclin E both regulates and is regulated by calpain 2, a protease associated with metastatic breast cancer phenotype. Cancer Res 65: 10700-10708.

Lim KL, Lim GG. 2011. K63-linked ubiquitination and neurodegeneration. Neurobiol Dis 43: 9-16.

Lin GN, Corominas R, Lemmens I, Yang X, Tavernier J, Hill DE, Vidal M, Sebat J, Iakoucheva LM. 2015. Spatiotemporal 16p11.2 protein network implicates cortical late mid-fetal brain development and KCTD13-Cul3-RhoA pathway in psychiatric diseases. Neuron 85: 742-754.

Lin Z, Li S, Feng C, Yang S, Wang H, Ma D, Zhang J, Gou M, Bu D, Zhang T et al. 2016. Stabilizing mutations of KLHL24 ubiquitin ligase cause loss of keratin 14 and human skin fragility. Nat Genet.

Litan A, Langhans SA. 2015. Cancer as a channelopathy: ion channels and pumps in tumor development and progression. Front Cell Neurosci 9: 86.

Loeb KR, Kostner H, Firpo E, Norwood T, Tsuchiya KD, Clurman BE, Roberts JM. 2005. A mouse model for cyclin E-dependent genetic instability and tumorigenesis. Cancer Cell 8: 35-47.

Louis-Dit-Picard H, Barc J, Trujillano D, Miserey-Lenkei S, Bouatia-Naji N, Pylypenko O, Schott J-J, Jeunemaitre X. 2012. KLHL3 mutations cause familial hyperkalemic hypertension by impairing ion transport in the distal nephron. Nature Genetics

44: 456-460.

Lu A, Pfeffer SR. 2013. Golgi-associated RhoBTB3 targets cyclin E for ubiquitylation and promotes cell cycle progression. J Cell Biol 203: 233-250. 
Lubec G, Sohn SY. 2003. RNA microarray analysis of channels and transporters in normal and fetal Down syndrome (trisomy 21) brain. J Neural Transm Suppl: 215-224.

Mailand N, Diffley JF. 2005. CDKs promote DNA replication origin licensing in human cells by protecting Cdc6 from APC/C-dependent proteolysis. Cell 122: 915-926.

Mani RS. 2014. The emerging role of speckle-type POZ protein (SPOP) in cancer development. Drug Discov Today 19: 1498-1502.

Marples D, Schroer TA, Ahrens N, Taylor A, Knepper MA, Nielsen S. 1998. Dynein and dynactin colocalize with AQP2 water channels in intracellular vesicles from kidney collecting duct. Am J Physiol 274: F384-394.

Mathew R, Seiler MP, Scanlon ST, Mao AP, Constantinides MG, Bertozzi-Villa C, Singer JD, Bendelac A. 2012. BTB-ZF factors recruit the E3 ligase cullin 3 to regulate lymphoid effector programs. Nature 491: 618-621.

McCormick JA, Ellison DH. 2011. The WNKs: Atypical Protein Kinases with Pleiotropic Actions. Physiology Reviews 91: 177-219.

McCormick JA, Yang CL, Zhang C, Davidge B, Blankenstein KI, Terker AS, Yarbrough B, Meermeier NP, Park HJ, McCully B et al. 2014. Hyperkalemic hypertensionassociated cullin 3 promotes WNK signaling by degrading KLHL3. J Clin Invest 124: 4723-4736.

McEvoy J, Kossatz U, Malek N, Singer J. 2007. Constitutive turnover of cylin E by Cul3 maintains quiescence. Molecular Cell Biology 27: 3651-3666.

Metzger MB, Hristova VA, Weissman AM. 2012. HECT and RING finger families of E3 ubiquitin ligases at a glance. J Cell Sci 125: 531-537.

Metzger MB, Pruneda JN, Klevit RE, Weissman AM. 2014. RING-type E3 ligases: master manipulators of E2 ubiquitin-conjugating enzymes and ubiquitination. Biochim Biophys Acta 1843: 47-60.

Mikaelsson E, Danesh-Manesh AH, Luppert A, Jeddi-Tehrani M, Rezvany MR, Sharifian RA, Safaie R, Roohi A, Osterborg A, Shokri F et al. 2005. Fibromodulin, an extracellular matrix protein: characterization of its unique gene and protein expression in B-cell chronic lymphocytic leukemia and mantle cell lymphoma. Blood 105: 4828-4835.

Minella AC, Loeb KR, Knecht A, Welcker M, Varnum-Finney BJ, Bernstein ID, Roberts JM, Clurman BE. 2008. Cyclin E phosphorylation regulates cell proliferation in hematopoietic and epithelial lineages in vivo. Genes Dev 22: 1677-1689.

Mishra S, Melino G, Murphy LJ. 2007. Transglutaminase 2 kinase activity facilitates protein kinase A-induced phosphorylation of retinoblastoma protein. The Journal of biological chemistry 282: 18108-18115.

Morimura N, Inoue T, Katayama K, Aruga J. 2006. Comparative analysis of structure, expression and PSD95-binding capacity of Lrfn, a novel family of neuronal transmembrane proteins. Gene 380: 72-83.

Nam J, Mah W, Kim E. 2011. The SALM/Lrfn family of leucine-rich repeat-containing cell adhesion molecules. Semin Cell Dev Biol 22: 492-498.

Nedvetsky PI, Tamma G, Beulshausen S, Valenti G, Rosenthal W, Klussmann E. 2009. Regulation of aquaporin-2 trafficking. Handb Exp Pharmacol: 133-157. 
Nishitani H, Lygerou Z. 2002. Control of DNA replication licensing in a cell cycle. Genes Cells 7: 523-534.

O'Shaughnessy KM. 2015. Gordon Syndrome: a continuing story. Pediatr Nephrol 30: 1903-1908.

Ohta A, Schumacher F-R, Mehellou Y, Johnson C, Knebel A, Macartney TJ, Wood NT, Alessi DR, Kurz T. 2013. The CUL3-KLHL3 E3 ligse complex mutated in Gordon's hypertension syndrome interacts with and ubiquitylates WNK isoforms: disease causing mutations in Klhle and WNK4 disrupt interaction. Biochemical Journal 451: 111-122.

Ohtsubo M, Roberts JM. 1993. Cyclin-dependent regulation of G1 in mammalian fibroblasts. Science (New York, NY) 259: 1908-1912.

Olesen ET, Fenton RA. 2017. Aquaporin-2 membrane targeting: still a conundrum. Am J Physiol Renal Physiol: ajprenal 0001002017.

Oliverio S, Amendola A, Di Sano F, Farrace MG, Fesus L, Nemes Z, Piredda L, Spinedi A, Piacentini M. 1997. Tissue transglutaminase-dependent posttranslational modification of the retinoblastoma gene product in promonocytic cells undergoing apoptosis. Molecular and cellular biology 17: 6040-6048.

Pardee AB. 1974. A restriction point for control of normal animal cell proliferation. Proc Natl Acad Sci U S A 71: 1286-1290.

Penkert J, Ripperger T, Schieck M, Schlegelberger B, Steinemann D, Illig T. 2016. On metabolic reprogramming and tumor biology: A comprehensive survey of metabolism in breast cancer. Oncotarget 7: 67626-67649.

Perez-Neut M, Shum A, Cuevas BD, Miller R, Gentile S. 2015. Stimulation of hERG1 channel activity promotes a calcium-dependent degradation of cyclin E2, but not cyclin E1, in breast cancer cells. Oncotarget 6: 1631-1639.

Petroski MD, Deshaies RJ. 2005. Function and regulation of cullin-RING ubiquitin ligases. Nat Rev Mol Cell Biol 6: 9-20.

Pickart CM, Rose IA. 1985. Functional heterogeneity of ubiquitin carrier proteins. The Journal of biological chemistry 260: 1573-1581.

Pintard L, Willems A, Peter M. 2004. Cullin-based ubiquitin ligases: Cul3-BTB complexes join the family. EMBO J 23: 1681-1687.

Pintard L, Willis JH, Willems A, Johnson JL, Srayko M, Kurz T, Glaser S, Mains PE, Tyers M, Bowerman B et al. 2003. The BTB protein MEL-26 is a substratespecific adaptor of the CUL-3 ubiquitin-ligase. Nature 425: 311-316.

Plafker KS, Singer JD, Plafker SM. 2009. The ubiquitin conjugating enzyme, UbcM2, engages in novel interactions with components of cullin-3 based E3 ligases. Biochemistry 48: 3527-3537.

Porter DC, Keyomarsi K. 2000. Novel splice variants of cyclin E with altered substrate specificity. Nucleic Acids Res 28: E101.

Porter DC, Zhang N, Danes C, McGahren MJ, Harwell RM, Faruki S, Keyomarsi K. 2001. Tumor-specific proteolytic processing of cyclin $\mathrm{E}$ generates hyperactive lower-molecular-weight forms. Molecular and cellular biology 21: 6254-6269. 
Presson AP, Yoon NK, Bagryanova L, Mah V, Alavi M, Maresh EL, Rajasekaran AK, Goodglick L, Chia D, Horvath S. 2011. Protein expression based multimarker analysis of breast cancer samples. BMC Cancer 11: 230.

Rath SL, Senapati S. 2014. Why are the truncated cyclin Es more effective CDK2 activators than the full-length isoforms? Biochemistry 53: 4612-4624.

Resnitzky D, Gossen M, Bujard H, Reed SI. 1994. Acceleration of the G1/S phase transition by expression of cyclins D1 and E with an inducible system. Molecular and cellular biology 14: 1669-1679.

Richardson HE, O'Keefe LV, Reed SI, Saint R. 1993. A Drosophila G1-specific cyclin E homolog exhibits different modes of expression during embryogenesis. Development 119: 673-690.

Ridley AJ. 2001. Rho proteins: linking signaling with membrane trafficking. Traffic 2: 303-310.

Rogers S, Wells R, Rechsteiner M. 1986. Amino acid sequences common to rapidly degraded proteins: the PEST hypothesis. Science (New York, NY) 234: 364368.

Rogers SW, Rechsteiner MC. 1986. Microinjection studies on selective protein degradation: relationships between stability, structure, and location. Biomed Biochim Acta 45: 1611-1618.

Said TK, Medina D. 1995. Cell cyclins and cyclin-dependent kinase activities in mouse mammary tumor development. Carcinogenesis 16: 823-830.

Schenkova K, Lutz J, Kopp M, Ramos S, Rivero F. 2012. MUF1/leucine-rich repeat containing 41 (LRRC41), a substrate of RhoBTB-dependent cullin 3 ubiquitin ligase complexes, is a predominantly nuclear dimeric protein. J Mol Biol 422: 659-673.

Scuderi R, Palucka KA, Pokrovskaja K, Bjorkholm M, Wiman KG, Pisa P. 1996. Cyclin $\mathrm{E}$ overexpression in relapsed adult acute lymphoblastic leukemias of B-cell lineage. Blood 87: 3360-3367.

Selvakumar P, Owens TA, David JM, Petrelli NJ, Christensen BC, Lakshmikuttyamma A, Rajasekaran AK. 2014. Epigenetic silencing of Na,K-ATPase beta 1 subunit gene ATP1B1 by methylation in clear cell renal cell carcinoma. Epigenetics $\mathbf{9}$ : 579-586.

Sherr CJ. 1994. G1 phase progression: cycling on cue. Cell 79: 551-555.

Sherr CJ, Roberts JM. 1999. CDK inhibitors: positive and negative regulators of G1phase progression. Genes Dev 13: 1501-1512.

Shibata S, Zhang J, Puthumana J, Stone KL, Lifton RP. 2013. Kelch-like 3 and Cullin 3 regulate electrolyte homeostasis via ubiquitination and deradation of WNK4. PNAS 110: 7838-7843.

Siddiqi S, Sussman MA. 2014. The heart: mostly postmitotic or mostly premitotic? Myocyte cell cycle, senescence, and quiescence. Can J Cardiol 30: 1270-1278.

Singer JD, Gurian-West M, Clurman B, Roberts JM. 1999. Cullin-3 targets cyclin E for ubiquitination and controls S phase in mammalian cells. Genes Dev 13: 23752387. 
Skowyra D, Craig KL, Tyers M, Elledge SJ, Harper JW. 1997. F-box proteins are receptors that recruit phosphorylated substrates to the SCF ubiquitin-ligase complex. Cell 91: 209-219.

Sparaneo A, Fabrizio FP, Muscarella LA. 2016. Nrf2 and Notch Signaling in Lung Cancer: Near the Crossroad. Oxid Med Cell Longev 2016: 7316492.

Spruck CH, Won KA, Reed SI. 1999. Deregulated cyclin E induces chromosome instability. Nature 401: 297-300.

Stogios PJ, Downs GS, Jauhal JJ, Nandra SK, Prive GG. 2005. Sequence and structural analysis of BTB domain proteins. Genome Biol 6: R82.

Strohmaier H, Spruck CH, Kaiser P, Won KA, Sangfelt O, Reed SI. 2001. Human F-box protein hCdc4 targets cyclin E for proteolysis and is mutated in a breast cancer cell line. Nature 413: 316-322.

Suzuki T, Seki S, Hiramoto K, Naganuma E, Kobayashi EH, Yamaoka A, Baird L, Takahashi N, Sato H, Yamamoto M. 2017. Hyperactivation of Nrf2 in early tubular development induces nephrogenic diabetes insipidus. Nat Commun 8: 14577.

Swiss VA, Casaccia P. 2010. Cell-context specific role of the E2F/Rb pathway in development and disease. Glia 58: 377-390.

Takata K, Matsuzaki T, Tajika Y, Ablimit A, Hasegawa T. 2008. Localization and trafficking of aquaporin 2 in the kidney. Histochem Cell Biol 130: 197-209.

Tamma G, Klussmann E, Maric K, Aktories K, Svelto M, Rosenthal W, Valenti G. 2001. Rho inhibits cAMP-induced translocation of aquaporin-2 into the apical membrane of renal cells. Am J Physiol Renal Physiol 281: F1092-1101.

Tao Y, Dai P, Liu Y, Marchetto S, Xiong WC, Borg JP, Mei L. 2009. Erbin regulates NRG1 signaling and myelination. Proc Natl Acad Sci U S A 106: 9477-9482.

Thalhammer A, Trinidad JC, Burlingame AL, Schoepfer R. 2009. Densin-180: revised membrane topology, domain structure and phosphorylation status. $J$ Neurochem 109: 297-302.

Tyers M, Jorgensen P. 2000. Proteolysis and the cell cycle: with this RING I do thee destroy. Curr Opin Genet Dev 10: 54-64.

Wadelin F, Fulton J, McEwan PA, Spriggs KA, Emsley J, Heery DM. 2010. Leucine-rich repeat protein PRAME: expression, potential functions and clinical implications for leukaemia. Mol Cancer 9: 226.

Wakabayashi M, Mori T, Isobe K, Sohara E, Susa K, Araki Y, Chiga M, Kikuchi E, Nomura N, Mori Y et al. 2013. Impaired KLHL3-mediated Ubiquitination of WNK4 Causes Human Hypertenison. Cell Reports 2013: 1-11.

Wakabayashi N, Itoh K, Wakabayashi J, Motohashi H, Noda S, Takahashi S, Imakado S, Kotsuji T, Otsuka F, Roop DR et al. 2003. Keap1-null mutation leads to postnatal lethality due to constitutive Nrf2 activation. Nat Genet 35: 238-245.

Wang T, Guo H, Xiong B, Stessman HA, Wu H, Coe BP, Turner TN, Liu Y, Zhao W, Hoekzema K et al. 2016. De novo genic mutations among a Chinese autism spectrum disorder cohort. Nat Commun 7: 13316.

Wang XD, Rosales JL, Magliocco A, Gnanakumar R, Lee KY. 2003. Cyclin E in breast tumors is cleaved into its low molecular weight forms by calpain. Oncogene 22: 769-774. 
Welcker M, Singer J, Loeb KR, Grim J, Bloecher A, Gurien-West M, Clurman BE, Roberts JM. 2003. Multisite phosphorylation by Cdk2 and GSK3 controls cyclin E degradation. Mol Cell 12: 381-392.

Westerlund M, Belin AC, Anvret A, Bickford P, Olson L, Galter D. 2008. Developmental regulation of leucine-rich repeat kinase 1 and 2 expression in the brain and other rodent and human organs: Implications for Parkinson's disease. Neuroscience 152: 429-436.

Wimuttisuk W, Singer JD. 2007. The Cullin3 ubiquitin ligase functions as a Nedd8bound heterodimer. Mol Biol Cell 18: 899-909.

Wimuttisuk W, West M, Davidge B, Yu K, Salomon A, Singer JD. 2014. Novel Cul3 binding proteins function to remodel E3 ligase complexes. BMC Cell Biol 15: 28.

Wingate H, Zhang N, McGarhen MJ, Bedrosian I, Harper JW, Keyomarsi K. 2005. The tumor-specific hyperactive forms of cyclin $\mathrm{E}$ are resistant to inhibition by p21 and p27. The Journal of biological chemistry 280: 15148-15157.

Won KA, Reed SI. 1996. Activation of cyclin E/CDK2 is coupled to site-specific autophosphorylation and ubiquitin-dependent degradation of cyclin E. EMBO J 15: 4182-4193.

Wu JH, Liu JH, Ko YC, Wang CT, Chung YC, Chu KC, Liu TT, Chao HM, Jiang YJ, Chen SJ et al. 2016. Haploinsufficiency of RCBTB1 is associated with Coats disease and familial exudative vitreoretinopathy. Hum Mol Genet 25: 1637-1647.

Wu X, Kong X, Luchsinger L, Smith BD, Xu Y. 2009. Regulating the activity of class II transactivator by posttranslational modifications: exploring the possibilities. Molecular and cellular biology 29: 5639-5644.

Xu L, Wei Y, Reboul J, Vaglio P, Shin TH, Vidal M, Elledge SJ, Harper JW. 2003. BTB proteins are substrate-specific adaptors in an SCF-like modular ubiquitin ligase containing CUL-3. Nature 425: 316-321.

Yang CL, Angell J, Mitchell R, Ellison DH. 2003. WNK kinases regulate thiazidesensitive $\mathrm{Na}-\mathrm{Cl}$ cotransport. J Clin Invest 111: 1039-1045.

Yoshimura SH, Iwasaka S, Schwarz W, Takeyasu K. 2008. Fast degradation of the auxiliary subunit of $\mathrm{Na}+/ \mathrm{K}+-\mathrm{ATP}$ ase in the plasma membrane of HeLa cells. $J$ Cell Sci 121: 2159-2168.

Zhang CS, Liu Q, Li M, Lin SY, Peng Y, Wu D, Li TY, Fu Q, Jia W, Wang X et al. 2015. RHOBTB3 promotes proteasomal degradation of HIFalpha through facilitating hydroxylation and suppresses the Warburg effect. Cell Res 25: 1025-1042.

Zhang DD, Lo SC, Cross JV, Templeton DJ, Hannink M. 2004. Keap1 is a redoxregulated substrate adaptor protein for a Cul3-dependent ubiquitin ligase complex. Molecular and cellular biology 24: 10941-10953.

Zhang P, Gao K, Tang Y, Jin X, An J, Yu H, Wang H, Zhang Y, Wang D, Huang $\mathrm{H}$ et al. 2014. Destruction of DDIT3/CHOP protein by wild-type SPOP but not prostate cancer-associated mutants. Hum Mutat.

Zheng N, Schulman BA, Song L, Miller JJ, Jeffrey PD, Wang P, Chu C, Koepp DM, Elledge SJ, Pagano M et al. 2002. Structure of the Cul1-Rbx1-Skp1-F boxSkp2 SCF ubiquitin ligase complex. Nature 416: 703-709. 